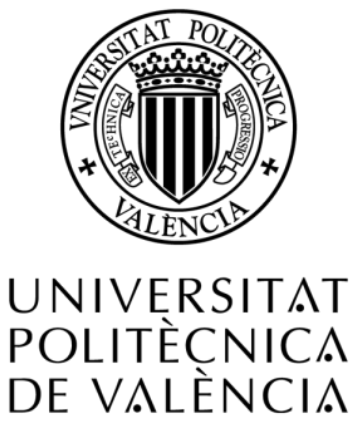

\title{
ANNOTATION MECHANISMS TO MANAGE DESIGN KNOWLEDGE IN COMPLEX PARAMETRIC MODELS AND THEIR EFFECTS ON ALTERATION AND REUSABILITY
}

\author{
A Dissertation \\ by \\ JORGE DORRIBO CAMBA
}

Submitted to the Doctoral School of

Universitat Politècnica de València

in partial fulfillment of the requirements for the degree of

\section{DOCTOR OF PHILOSOPHY}

under the supervision of Dr. Manuel Contero González

Major Subject: Design, Manufacturing and Management of Industrial Projects

September 2014 



\section{ACKNOWLEDGEMENTS}

I would like to express my deepest gratitude and appreciation to my advisor, Professor Dr. Manuel Contero, for his expert guidance, support, and valuable suggestions and critiques to this work. Thank you for accepting me as your $\mathrm{PhD}$ student and giving me the opportunity to work with you. I honestly could not have asked for a better advisor.

I also thank my family for their love, patience, and support throughout this process and for letting me steal their valuable time to work on this dissertation. It is my hope that their sacrifice has finally paid off. 


\section{ABSTRACT}

Despite the evolution of technologies intended to facilitate and optimize product development processes and foster collaboration, effective reuse of 3D models remains one of the biggest challenges in the area of ComputerAided Design. Whether a manufacturer is designing a new commercial aircraft or a household appliance, engineering teams often start with existing designs and adapt them to new cases, rather than designing every product from scratch. Nevertheless, CAD model reuse is not effectively supported by conventional CAD packages, as much of the burden related to reusability lies on the CAD user.

It has been shown that CAD model reusability largely depends on a proper definition and communication of the geometric design intent, which are usually expressed implicitly within the CAD model. This implicit representation makes it difficult for $\mathrm{CAD}$ users interacting with a $\mathrm{CAD}$ model to understand how and why the model was created in a specific manner. This is especially true for models being reworked by designers that are not the original creators of the models. The inability to understand and modify existing CAD models negatively affects reusability and hinders the collaborative design process. The problem becomes more relevant in modelbased engineering environments, where 3D models are used as the main shared data source for all engineering activities throughout the product life cycle.

Recent research has explored the potential of 3D annotations as tools to carry design intent information. The focus of this doctoral research is to study the effectiveness of 3D CAD annotation techniques to support the explicit representation and communication of design intent, and to analyze the impact of these techniques in the alteration and reutilization of $3 \mathrm{D}$ models in a product design context.

Literature shows that a good and structured methodology is an essential step to create parametric models that are reusable and can be altered easily. However, when models reach a certain level of complexity in terms of number of features and interdependencies, additional mechanisms must be established so design intent can be communicated effectively in an explicit manner. In this regard, a comparative study was conducted to determine the complexity of three professionally accepted modeling methodologies. These methodologies represent a group of well tested and documented methodologies that are currently available to the public. An efficient 
modeling methodology can provide a competitive advantage in industrial settings so companies are often reluctant to make this information public. An experimental software system was developed to examine the internal structure of parametric CAD models according to a set of complexity metrics.

Recent studies have suggested the use of 3D annotations as a method to embed design information in the model's geometry and make part of the design knowledge explicitly available. An exploratory study was performed to examine the formal annotation practices defined by model-based standards such as ASME Y14.41-2012 and ISO 16792:2006, and their implementation in current CAD systems. A series of experimental studies were conducted to evaluate the effectiveness of existing textual annotation mechanisms defined by current standards and analyze their impact in model alteration tasks. Effectiveness is analyzed in terms of the ability of the annotation to communicate design information to CAD users so CAD model alterations are performed correctly and efficiently. Results show that annotated models provide significant benefits when performing activities that require a direct manipulation of the model's geometry, but more advanced annotation mechanisms than those defined by current standards are necessary.

An extended annotation model that builds on current standards is introduced. The architecture of a new software system to communicate geometric design intent information explicitly by overloading and extending the scope of the current annotation instruments is presented. This system introduces a new broader type of model annotation where design information is represented both internally within the 3D model and externally, on a separate repository; and a new Graphic User Interface (GUI) embedded within the CAD environment to support the interaction of CAD users (designers and engineers) with the information. Integration of the proposed solution with existing Product Lifecycle Management systems as well as additional tools such as an annotation history module and an annotation-based communication tool for collaborative environments are presented. Experimental results show a statistically significant benefit of using the proposed software architecture in terms of CAD alteration times and correctness of the models after modifications in different design scenarios, suggesting the use of this annotation model as a valuable approach to improve design intent communication.

Keywords: 3D annotations, design intent, CAD model reusability, modelbased enterprise, reusable parametric models. 


\section{RESUMEN}

A pesar de la constante evolución de las tecnologías destinadas a facilitar y optimizar los procesos de desarrollo de nuevos productos, la reutilización efectiva de modelos tridimensionales sigue siendo uno de los mayores retos en el área de Diseño Asistido por Ordenador (CAD, por sus siglas en inglés). En la gran mayoría de los casos, el desarrollo de nuevos productos comienza a partir de diseños existentes que son modificados y/o adaptados a nuevas situaciones y requerimientos. Solamente en contadas ocasiones se diseña un producto desde cero. Sin embargo, la reutilización de modelos CAD no está soportada de manera eficiente por los paquetes CAD convencionales, ya que gran parte de la carga de trabajo relacionada con la reutilización recae sobre el usuario de CAD.

Se ha demostrado que la reutilización de modelos CAD depende en gran medida de una adecuada definición y comunicación de la intención de diseño, que normalmente se expresa implícitamente dentro del propio modelo CAD. Esta representación implícita hace que sea difícil para los usuarios CAD que interactúan con un modelo comprender cómo y por qué dicho modelo fue creado de cierta manera. Esto es especialmente notable en modelos que necesitan ser modificados por diseñadores que no son los creadores originales de los modelos. La dificultad para entender y modificar modelos CAD existentes afecta negativamente a la reutilización y obstaculiza el proceso de diseño colaborativo. El problema de la reutilización de modelos CAD se hace más notable en entornos de ingeniería basados en modelos (MBE, por sus siglas en inglés), ya que en estos entornos los modelos 3D se utilizan como la principal fuente de información compartida para todas las actividades del ciclo de vida del producto.

En estudios recientes se ha explorado el potencial de las anotaciones 3D como herramientas para almacenar información referente a la intención de diseño. Esta investigación doctoral se centra en el estudio de técnicas de anotación de modelos CAD paramétricos y su viabilidad para apoyar la representación y comunicación explícita de la intención de diseño ("design intent"). Para ello, se presenta un análisis del impacto de estas técnicas en la edición y la reutilización de modelos $3 \mathrm{D}$ en un contexto de diseño de producto así como una propuesta de "anotación extendida" basada en anotaciones estándar que mejora las prestaciones de las herramientas CAD existentes. 
La revisión del estado del arte muestra que el uso de una metodología de modelado bien estructurada es un paso esencial para crear modelos paramétricos que sean fácilmente editables y reutilizables. En este sentido, como parte de esta investigación se realizó un estudio comparativo de las tres metodologías de modelado paramétrico más representativas y aceptadas profesionalmente. Estas metodologías representan un grupo de técnicas bien validadas y documentadas que se encuentran disponibles públicamente. Una metodología de modelado eficiente puede proporcionar una ventaja competitiva en entornos industriales. Por lo tanto, muchas organizaciones son reacias a hacer pública esta información. Para dicho estudio, se desarrolló una herramienta experimental software que examina la estructura interna de los modelos CAD paramétricos de acuerdo a un conjunto de métricas de complejidad.

En algunos estudios recientes se ha sugerido el uso de anotaciones CAD como método para integrar la información de diseño en la propia geometría del modelo y hacer que parte del conocimiento esté disponible de forma explícita. Inicialmente, para esta investigación se examinaron las prácticas formales de anotación definidas por normas de ingeniería basada en modelos (ASME Y14.41-2012 e ISO 16792:2006), y su implementación en sistemas CAD actuales a través de módulos PMI (Product Manufacturing Information). Se han realizado una serie de estudios experimentales para evaluar la eficacia de los mecanismos de anotación existentes definidos por las normas vigentes y analizar su impacto en tareas de edición de modelos. La eficacia se analiza en términos de la capacidad de la anotación para comunicar la información de diseño a usuarios CAD de modo que las alteraciones en el modelo se realizan de manera correcta y eficiente. Los resultados revelan que los modelos anotados con información de diseño proporcionan beneficios significativos en situaciones que requieren una manipulación directa de la geometría del modelo. Sin embargo, las herramientas de anotación actuales son limitadas en cuanto a gestión de la información de intención de diseño. Es necesario, por lo tanto, el desarrollo de nuevos y mejores mecanismos de anotación, más avanzados que los definidos por los estándares actuales.

Como contribución, se presenta un modelo de anotación extendida basado en las normas actuales así como la arquitectura de un nuevo sistema software para comunicar de manera explícita la intención de diseño dentro del modelo CAD. El modelo propuesto se basa en un tipo extendido de anotación, donde la información de diseño está representado tanto a nivel interno dentro del modelo 3D como en un repositorio externo de información. Se introducen, además, una nueva interfaz gráfica de usuario (GUI) integrada dentro del 
entorno de modelado para apoyar la interacción de los usuarios CAD (diseñadores e ingenieros) con la información, una arquitectura de integración de la solución propuesta con plataformas de gestión del ciclo de vida del producto (PLM, por sus siglas en inglés), y dos módulos relacionados: un historial de anotaciones y una herramienta de comunicación basada en la anotación para entornos de colaboración. Los resultados experimentales muestran los beneficios de la arquitectura propuesta en términos de tiempos de alteración y validez de los modelos después de realizar modificaciones en diferentes escenarios, lo que confirma su valor como herramienta de comunicación de intención de diseño. 


\section{RESUM}

A pesar de la constant evolució de les tecnologies destinades a facilitar i optimitzar els processos de desenrotllament de nous productes, la reutilització efectiva de models tridimensionals continua sent un dels majors reptes en l'àrea de Disseny Assistit per Ordinador (CAD, per les seues sigles en anglés). En la gran majoria dels casos, el desenrotllament de nous productes comença a partir de dissenys existents que són modificats y/o adaptats a noves situacions i requeriments. Només en comptades ocasions es dissenya un producte des de zero. No obstant això, la reutilització de models CAD no està suportada de manera eficient pels paquets CAD convencionals, ja que gran part de la càrrega de treball relacionada amb la reutilització recau sobre l'usuari de CAD.

S'ha demostrat que la reutilització de models CAD depén en gran manera d'una adequada definició i comunicació de la intenció de disseny, que normalment s'expressa implícitament dins del propi model CAD. Esta representació implícita fa que siga difícil per als usuaris CAD que interactuen amb un model comprendre com i per què el dit model va ser creat d'una certa manera. Açò és especialment notable en models que necessiten ser modificats per dissenyadors que no són els creadors originals dels models. La dificultat per a entendre i modificar models CAD existents afecta negativament la reutilització i obstaculitza el procés de disseny col-laboratiu. El problema de la reutilització de models CAD es fa més notable en entorns d'enginyeria basats en models (MBE, per les seues sigles en anglés), ja que en estos entorns els models 3D s'utilitzen com la principal font d'informació compartida per a totes les activitats del cicle de vida del producte.

En estudis recents s'ha explorat el potencial de les anotacions 3D com a ferramentes per a emmagatzemar informació referent a la intenció de disseny. Esta investigació doctoral se centra en l'estudi de tècniques d'anotació de models CAD paramètrics i la seua viabilitat per a recolzar la representació i comunicació explícita de la intenció de disseny ("design intent"). Per a això, es presenta una anàlisi de l'impacte d'estes tècniques en l'edició i la reutilització de models 3D en un context de disseny de producte així com una proposta d'"anotació estesa" basada en anotacions estàndard que millora les prestacions de les ferramentes CAD existents.

La revisió de l'estat de l'art mostra que l'ús d'una metodologia de modelatge ben estructurada és un pas essencial per a crear models paramètrics que 
siguen fàcilment editables i reutilitzables. En este sentit, com a part d'esta investigació es va realitzar un estudi comparatiu de les tres metodologies de modelatge paramètric més representatives i acceptades professionalment. Estes metodologies representen un grup de tècniques ben validades $\mathrm{i}$ documentades que es troben disponibles públicament. Una metodologia de modelatge eficient pot proporcionar un avantatge competitiu en entorns industrials. Per tant, moltes organitzacions són poc inclinades a fer pública esta informació. Per a això, es va desenrotllar una ferramenta experimental software que examina l'estructura interna dels models CAD paramètrics d'acord amb un conjunt de mètriques de complexitat. Una vegada que els models aconseguixen un cert nivell de complexitat quant a nombre d'interdependències, les bones pràctiques de modelatge són insuficients i es necessiten establir mecanismes addicionals perquè la intenció de disseny es puga comunicar eficientment.

En alguns estudis recents s'ha suggerit l'ús d'anotacions CAD com a mètode per a integrar la informació de disseny en la pròpia geometria del model i fer que part del coneixement estiga disponible de forma explícita. Inicialment, per a esta investigació es van examinar les pràctiques formals d'anotació definides per normes d'enginyeria basada en models (ASME Y14.41-2012 i ISO 16792:2006), i la seua implementació en sistemes CAD actuals a través de mòduls PMI (Product Manufacturing Information). S'han realitzat una sèrie d'estudis experimentals per a avaluar l'eficàcia dels mecanismes d'anotació existents definits per les normes vigents i analitzar el seu impacte en tasques d'edició de models. L'eficàcia s'analitza en termes de la capacitat de l'anotació per a comunicar la informació de disseny a usuaris CAD de manera que les alteracions en el model es realitzen de manera correcta $\mathrm{i}$ eficient. Els resultats revelen que els models anotats amb informació de disseny proporcionen beneficis significatius en situacions que requerixen una manipulació directa de la geometria del model. No obstant això, les ferramentes d'anotació actuals són limitades quant a gestió de la informació d'intenció de disseny. És necessari, per tant, el desenrotllament de nous i millors mecanismes d'anotació, més avançats que els definits pels estàndards actuals.

Com a contribució, es presenta un model d'anotació estesa basat en les normes actuals així com l'arquitectura d'un nou sistema software per a comunicar de manera explícita la intenció de disseny dins del model CAD. El model proposat es basa en un tipus estés d'anotació, on la informació de disseny està representada tant a nivell intern dins del model 3D com en un repositori extern d'informació. S'introduïxen, a més, una nova interfície gràfica d'usuari (GUI) integrada dins de l'entorn de modelatge per a recolzar 
la interacció dels usuaris CAD (dissenyadors i enginyers) amb la informació, una arquitectura d'integració de la solució proposada amb plataformes de gestió del cicle de vida del producte (PLM, per les seues sigles en anglés) , i dos mòduls relacionats: un historial d'anotacions i una ferramenta de comunicació basada en l'anotació per a entorns de col•laboració. Els resultats experimentals mostren els beneficis de l'arquitectura proposada en termes de temps d'alteració i validesa dels models després de realitzar modificacions en diferents escenaris, la qual cosa confirma el seu valor com a ferramenta de comunicació d'intenció de disseny. 


\section{NOMENCLATURE}

2D Two-Dimensional

3D Three-Dimensional

ANSI American National Standards Institute

API Application Programming Interface

CAD Computer-Aided Design

CAM Computer-Aided Manufacturing

CSCW Computer Supported Collaborative Work

DSM Dependency Structure Matrix

GD\&T Geometric Dimensioning and Tolerancing

ISO International Organization for Standardization

MBD Model-Based Definition

MBE Model-Based Engineering

NIST National Institute of Standards and Technology

NPD New Product Development

PDM Product Data Management

PLM Product Lifecycle Management

PMI Product Manufacturing Information

XML eXtensible Markup Language 


\section{RELATED PUBLICATIONS}

The following refereed papers have been published (or are currently under review) as a result of this doctoral research.

\section{Journal Articles}

- Camba, J., Contero, M. (2014) "Assessing the Impact of Geometric Design Intent Annotations on Parametric Model Alteration Activities" Computers in Industry (under review)

- Camba, J., Contero, M., Johnson, M., Company, P. "Extended 3D Annotations as a New Mechanism to Explicitly Communicate Geometric Design Intent and Increase CAD Model Reusability" Computer-Aided Design, vol. 57, pp. 61-73, 2014. DOI: 10.1016/j.cad.2014.07.001.

\section{Conference Proceedings}

- Camba, J., Cosin, A., Contero, M., 2014, “An Evaluation of Formal Strategies to Create Stable and Reusable Parametric Feature-Based 3D Models.” ASME 2014 International Mechanical Engineering Congress and Exposition, IMECE 2014. Montreal, Quebec, Canada, 2014. Paper No. IMECE2014-37859 (accepted)

- Camba, J., Contero, M., Salvador-Herranz, G., 2014, "Implementation Challenges of Annotated 3D Models in Collaborative Design Environments" Lecture Notes in Computer Science 8683, pp. 222-229. Springer International Publishing Switzerland (2014). 11th International Conference on Cooperative Design, Visualization, and Engineering, Seattle, WA.

- Camba, J., Contero, M., Otey, J., Company, P., 2014, "Explicit Communication of Geometric Design Intent in CAD: Evaluating Annotated Models in the Context of Reusability.” ASME 2014 International Design and Engineering Technical Conferences \& Computers and Information in Engineering Conference, IDETC/CIE 2014. Paper No. DETC2014-34527.

- Camba J., Contero M., Johnson, M., 2014, "Management of Visual Clutter in Annotated 3D CAD Models: A Comparative Study." Lecture Notes in Computer Science 8518, pp. 405-416. 16th International Conference on Human-Computer Interaction, HCI. Crete, Greece. June 22-27, 2014.

- Camba, J., Contero, M., 2014, "Integration of Model-Based Design Annotations in Product Lifecycle Management Systems to Facilitate 
Design Intent Communication." Proceedings of the 10th International Symposium on Tools and Methods of Competitive Engineering, TMCE 2014. Budapest, Hungary. May 19-23, 2014. pp.1067-1076.

- Camba, J., Contero, M., Salvador-Herranz, G., 2014, "Speak with the Annotator: Promoting Interaction in a Knowledge-Based CAD Environment Built on the Extended Annotation Concept." Proceedings of the 18th IEEE International Conference on Computer Supported Cooperative Work in Design, CSCWD 2014. Hsinchu, Taiwan. May 21-23, 2014. pp.196-201.

- Camba, J., Alducin-Quintero, G., Perona, P., Contero, M., 2013, "Enhancing Model Reuse through 3D Annotations: A Theoretical Proposal for an Annotation-Centered Design Intent and Design Rationale Communication." Proceedings of the ASME 2013 International Mechanical Engineering Congress and Exposition, IMECE 2013. San Diego, CA. November 13-21, 2013. Paper No. IMECE2013-64595, pp. V012T13A010; doi:10.1115/IMECE2013-64595. 


\section{CONTENTS}

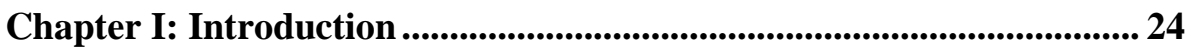

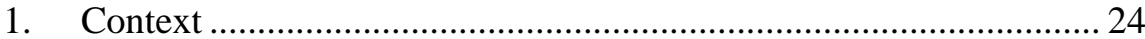

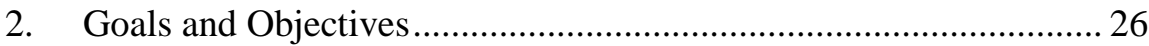

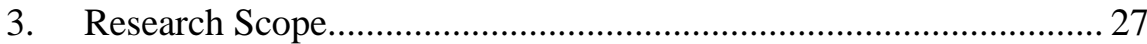

4. Contribution to Knowledge ............................................................ 29

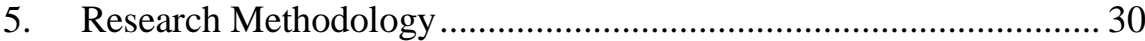

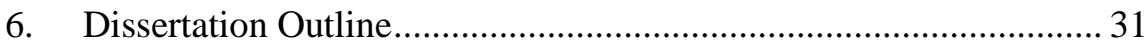

Chapter II: Literature Review ........................................................................ 33

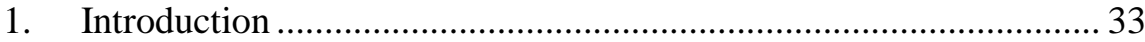

2. $\quad$ Model-Based Engineering (MBE) ................................................. 33

2.1. Standards supporting Model-Based Engineering ..................... 38

2.2. Product and Manufacturing Information in CAD Systems ...... 41

3. CAD Quality and Reusability ............................................................. 43

4. CAD Modeling Strategies for Reusability ....................................... 45

4.1. Delphi's Horizontal Modeling................................................... 47

4.2. Explicit Reference Modeling...................................................... 48

4.3. Resilient Modeling Strategy .................................................. 52

5. Data, Information, and Knowledge ................................................ 54

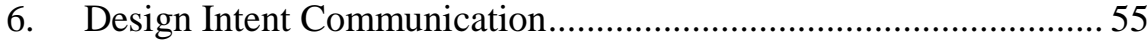

7. Annotations in Engineering Design................................................. 58

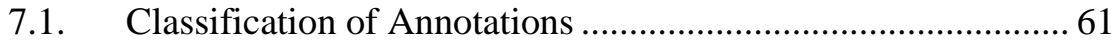

7.2. Annotations in Software Development …………………........ 65

7.3. State of the Art in 3D Annotation................................................ 67

7.4. The Problem of Visual Clutter .................................................... 69 
8. Collaborative Engineering and Computer Supported

Cooperative Work

8.1. Multimedia Supported Communication Technology for Collaborative Design 74

Chapter III: Parametric CAD Modeling Practices ........................................ 76

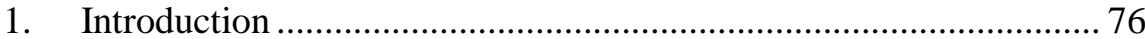

2. Approaches to 3D CAD Solid Modeling........................................... 77

2.1. Parametric Modeling vs. Direct Modeling ................................ 77

3. Feature-Based Parametric Solid Modeling ......................................... 80

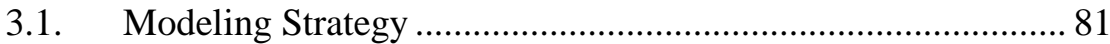

3.2. Modeling Example ................................................................ 82

4. Internal Representation of Parametric CAD models .......................... 84

4.1. Complexity Metrics ................................................................ 86

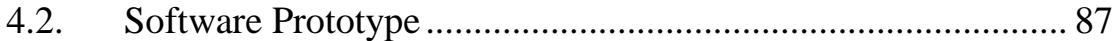

5. Case Study: A Comparison of Modeling Methodologies................... 89

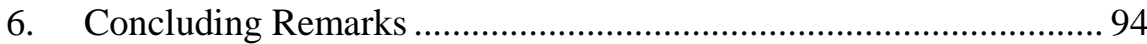

Chapter IV: Communication using Design Annotations .........................97

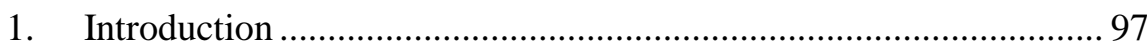

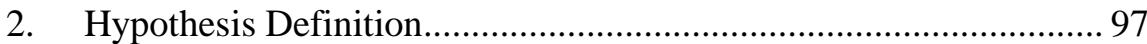

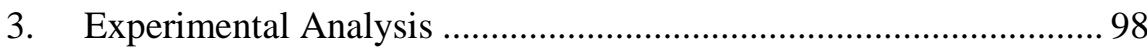

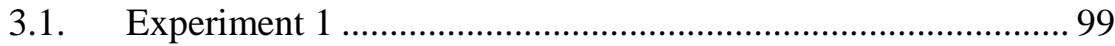

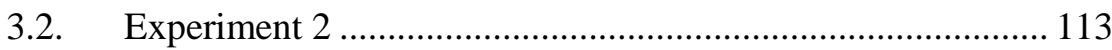

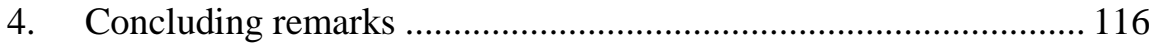

Chapter V: Extended Design Annotations ................................................ 118

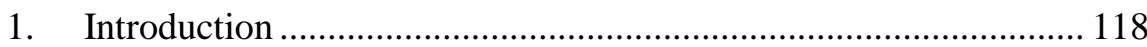

2. Implementation Challenges ......................................................... 118 
2.1. Annotation Storage .................................................................. 119

2.2. Annotation Content ............................................................. 119

2.3. Annotation Interface .............................................................. 120

2.4. Annotation Visualization...................................................... 120

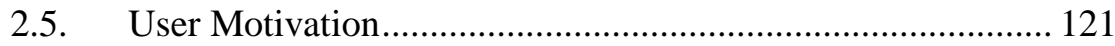

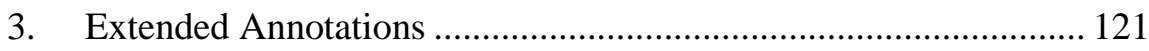

3.1. Annotation Storage .............................................................. 125

3.2. Annotation Content .............................................................. 128

3.3. Annotation Interface .......................................................... 129

3.4. Annotation Visualization...................................................... 132

3.5. User Motivation.................................................................... 133

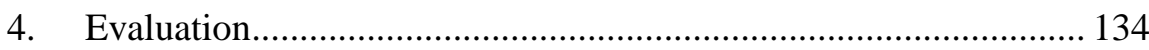

4.1. Experiments 1 and 2: User performance .............................. 134

4.2. Experiment 3: Visual Clutter................................................ 138

5. Concluding Remarks ............................................................... 143

Chapter VI: Integration in Collaborative Environments ....................... 144

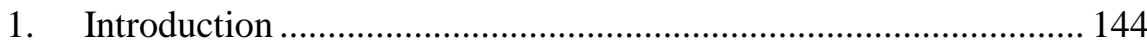

2. Product Lifecycle Management Systems ………........................... 144

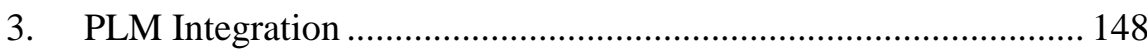

4. Annotation History ....................................................................... 150

5. Video Conferencing Component.................................................. 153

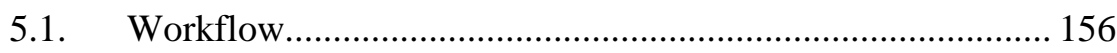

6. Concluding Remarks ………………………........................... 157

Chapter VII: Conclusions and Future Work.......................................... 159

1. Summary of Achievements .......................................................... 159

2. Contribution to Knowledge .......................................................... 161 
2.1. Understanding parametric model complexity......................... 162

2.2. Determining the effects of annotations in design intent

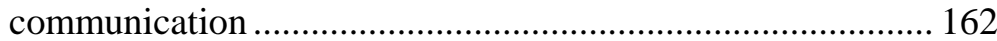

2.3. Extended annotations model and development of software prototype.

2.4. Connection of extended annotation model to other communication tools in collaborative design environments .. 164

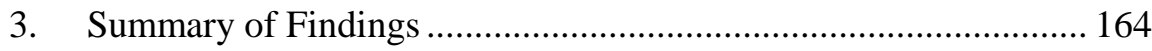

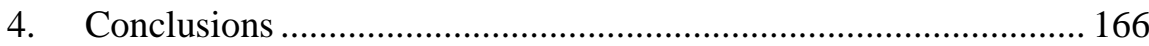

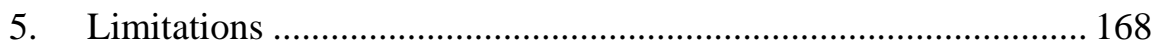

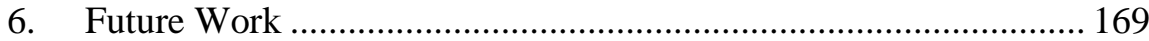

6.1. CAD Model Complexity ......................................................... 169

6.2. Extended Annotation Processing............................................. 170

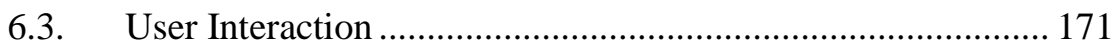

6.4. Further Experiments ........................................................... 171

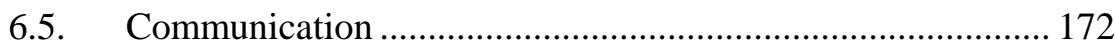

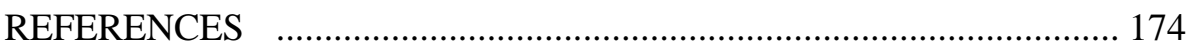

APPENDIX A: Part Alteration. Activity 1 ................................................. 189

APPENDIX B: Part Alteration. Activity 2 ............................................... 191

APPENDIX C: Experiments 1 and 2 ..................................................... 193 


\section{LIST OF FIGURES}

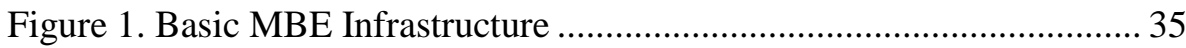

Figure 2. MBE Levels defined by DOD Engineering Drawing

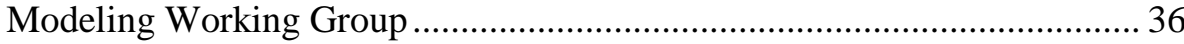

Figure 3. MBE relevant standards by area. .................................................. 39

Figure 4. Annotated models from ASME 14.41 and ISO 16792 ................. 40

Figure 5. Horizontal modeling strategy ........................................................ 47

Figure 6. Classification of modeling operations and

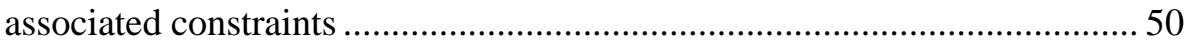

Figure 7. Modeling operations based on explicit management of

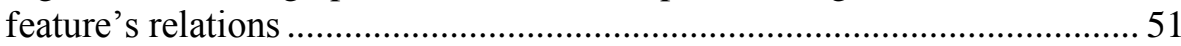

Figure 8. Sample design tree with proper RMS naming .............................. 54

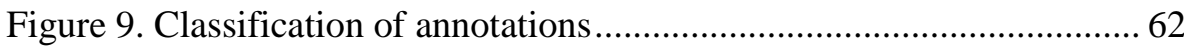

Figure 10. Visual clutter caused by annotations............................................ 71

Figure 11. Sample design tree in DS SolidWorks $®$.................................... 81

Figure 12. Graph representation and DSM of flange model ....................... 86

Figure 13. SolidWorks ${ }^{\circledR}$ module to visualize and process DSM.................. 88

Figure 14. Complex model and related information from module................ 88

Figure 15. Part used for comparison of modeling methodologies................. 89

Figure 16. Design trees according to the different methodologies ................ 90

Figure 17. DSMs of the design trees for the methodologies used

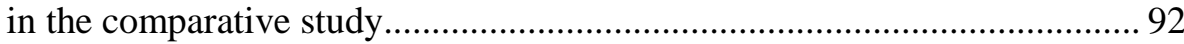

Figure 18. Graph representation of the design trees for the methodologies used in the comparative study............................................. 93

Figure 19. Model provided to participants for activity 1.......................... 100

Figure 20. Creation process of original model for activity 1 ...................... 100

Figure 21. Sequence of alterations requested for activity 1 ...................... 101

Figure 22. Basic approaches for Alteration 1 ............................................ 102 
Figure 23. Model provided to participants for activity 2........................... 105

Figure 24. Creation process of original model for activity 2..................... 106

Figure 25. Sequence of alterations requested for activity 2 ..................... 106

Figure 26. Two approaches to fillet creation............................................. 108

Figure 27. Feature fillets (incorrect) vs. Sketch fillets ................................ 109

Figure 28. Modeling approaches to new vertical connector....................... 112

Figure 29. Model provided to participants for experiment 2..................... 113

Figure 30. First alteration causes unwanted effect ...................................... 114

Figure 31. Possible solutions to design problem ....................................... 114

Figure 32. Information configuration of a 3D annotated model................. 122

Figure 33. Proposed Extended Annotation Model ...................................... 123

Figure 34. Components of Extended Annotation Model.............................. 123

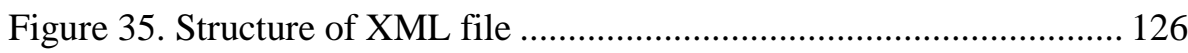

Figure 36. XML representation of extended annotations .......................... 127

Figure 37. Enhanced Entity-Relationship (EER) database model

for extended annotation system........................................................... 128

Figure 38. Annotated example …………………….............................. 129

Figure 39. Annotation synchronization ..................................................... 130

Figure 40. Example of annotated model and software prototype............... 131

Figure 41. External HTML document linked to an annotation ................... 132

Figure 42. Example of interactive visualization of annotations ................. 133

Figure 43. Average time improvements with annotation manager ............ 138

Figure 44. PLM in the context of the collaborative

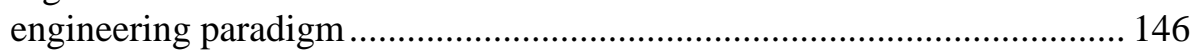

Figure 45. PDM structure ................................................................... 147

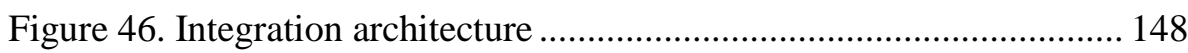

Figure 47. Enhanced Entity-Relationship (EER) database model for extended annotation system with history module................................ 151

Figure 48. Accessing history module ……….......................................... 151 
Figure 49. Annotation history records..................................................... 152

Figure 50. System architecture with video conferencing module .............. 155

Figure 51. CAD interface with video conferencing module ...................... 155

Figure 52. Contacting annotator .......................................................... 157 


\section{LIST OF TABLES}

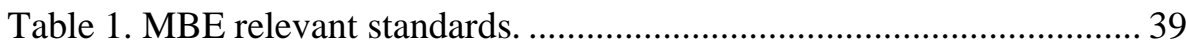

Table 2. Support of standards in commercial PMI modules ........................ 42

Table 3. Challenges and responses to CAD model reusability...................... 44

Table 4. Feature groups defined by the Resilient Modeling Strategy .......... 53

Table 5. Annotations in Model-Based standards............................................. 60

Table 6. Advantages and disadvantages of mark-up strategies ..................... 64

Table 7. Source code documentation example (from Wikipedia) ................. 66

Table 8. Summary of 3D annotation approaches (Target: 3D models) ........ 69

Table 9. Parametric Modeling vs. Direct Modeling ...................................... 79

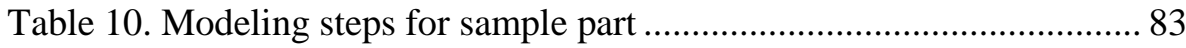

Table 11. Hole alteration steps for sample part ............................................ 84

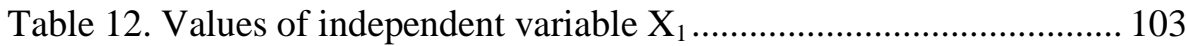

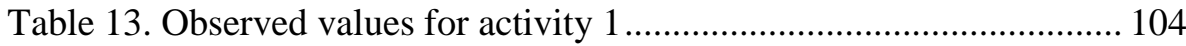

Table 14. Observed values for activity 2 (fillet creation) ............................ 109

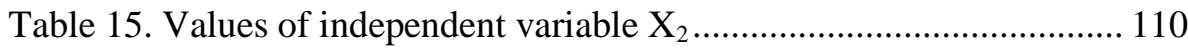

Table 16. Observed values for activity 2 (outlet creation) .......................... 111

Table 17. Observed values for activity 2 (new connector)......................... 113

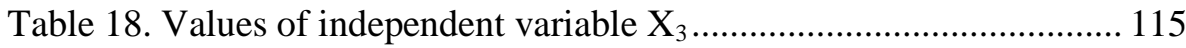

Table 19. Observed values for experiment 2 …..................................... 116

Table 20. T-test comparison of time (s) required to answer questions....... 136

Table 21. User Satisfaction Questionnaire and Results............................... 137

Table 22. Questions and relevant annotations with explicit information to answer correctly .............................................................. 140

Table 23. Annotation mechanisms and descriptions .................................. 140

Table 24. Question completion time by group and statistical

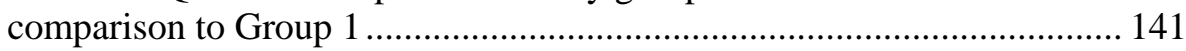

Table 25. Total time for all questions data comparison ............................ 142 



\section{Chapter I \\ Introduction}

\section{Context}

Engineering and product design are intellectually intensive and multidisciplinary activities that require constant exploring, iterating, optimizing, and testing until an efficient solution is designed and built. These activities should be agile and well-organized to provide enough flexibility for adapting to constantly evolving markets [Robertson \& Allen, 1993]. Three-dimensional Computer-Aided Design (CAD) software used in combination with sophisticated information management systems have become essential instruments in all engineering disciplines to guarantee the delivery of truly innovative products in a timely and cost effective manner.

In a linear step-by-step design process, where each piece of a system is designed one at a time, one after the other, integration and interfaces between different components are relatively simple to define. However, modern approaches for developing large and complex integrated products typically involve many individuals who work independently yet in parallel with others as part of a vast network of carefully orchestrated engineering and manufacturing efforts. This type of design scenario is time consuming, demanding, and error-prone, unless all activities are properly planned and effective strategies are correctly established [Chang et al., 1999; Herron, 2013].

From an industrial standpoint, history-based parametric CAD technology is currently a mature and commonly deployed technology that offers a 
methodical and sophisticated functionality to create intricate geometry. In addition to the obvious benefits of speed and the ability to create complex three dimensional CAD models with relative ease, more flexible and reusable designs can be achieved with parametric modeling systems [Bodein et al., 2013]. Flexibility and reusability of design elements are, in fact, critical pieces to accelerate design, improve quality, and guarantee success in New Product Development (NPD) processes. Rather than designing products from scratch every time, a large percentage of designs are reused to a certain extent to facilitate future development of products that are similar in function and shape [Jackson \& Buxton, 2007].

In today's industry, where engineering design relies heavily on digital product representations, reusing designs requires the effective reuse of threedimensional CAD models. The role of CAD models in the product lifecycle has gained more and more significance with the introduction of collaborative and model-based schemes. An increasing number of companies are adopting the Model-Based Engineering (MBE) paradigm, an approach to product development and manufacturing that uses digital models to drive all engineering activities. In this paradigm, CAD models become the central point around which all engineering processes revolve. MBE provides practical opportunities for efficiency and effectiveness in product development, particularly in the area of reusability [Lubell et al., 2012].

The potential benefits of history-based parametric CAD systems in terms of reusability, however, are far from what is expected by end users and claimed by software vendors. The ability to alter and reuse CAD models largely relies on efficient modeling strategies and proper understanding of geometric design intent rather than technology itself [Bodein et al., 2014].

Identifying the factors involved in defining the most efficient modeling approach to a particular design challenge is a difficult task. Since a virtually unlimited number of modeling strategies can be selected, determining the best method that ensures maximum flexibility and reusability largely depends on the designer's previous experience and good judgment [Hartman, 2005]. Although many companies define their own internal CAD modeling guidelines (often based on their own experts' knowledge), there is a limited amount of published information about effective modeling procedures. Experts' knowledge is often comprised of various types of knowledge, including explicit (formal policies, procedures, and best practices), tacit (practical experiences and shared values) and implicit (knowledge that is implied by or inferred from observable behavior or 
performance) [Guerra \& Young, 2006; Guerra-Zubiaga \& Young, 2008; Guerra \& Young, 2008]. In the context of this research, only knowledge derived from geometric design intent information is considered. This information is applicable to the geometry and structure of a specific CAD model.

In professional environments, efficient design methodologies and best practices may lead to important competitive advantages. For this reason, this type of information is often protected or only released in the form of patents [Landers \& Khurana, 2004]. As a result, formal modeling for reusability is a particular research area that is currently underdeveloped.

The second requisite to achieve reusability is the implementation of knowledge management enabling methods. There is evidence of the necessity to generate mechanisms to facilitate the information transfer throughout the entire product's lifecycle [Chandrasegaran et al., 2013]. In a CAD modeling context, this need typically translates to communication of the modeling strategy. Users working with a CAD model need to understand the reasons behind the modeling decisions. They need to know how and why the model was created in a specific manner, i.e., they need to understand its design intent [Iyer \& Mills, 2006]. This information is typically conveyed implicitly within the CAD model in the form of relations between features of the model, which are typically displayed as a design tree or history tree in the interface of the parametric modeling software.

The motivation of this work is to examine parametric modeling from a model complexity and reusability perspective and establish a novel mechanism based on 3D annotations to explicitly express design intent information in complex 3D CAD models to ultimately facilitate design communication. The proposed mechanism is presented as a framework where other engineering and communication tools can be integrated.

\section{Goals and Objectives}

The goal of this research is to analyze parametric model complexity in terms of reusability and communication of design information and to study annotation-based strategies as viable tools to explicitly represent and manage this type of information.

The general hypothesis can be stated as: "A significant part of the design information that is generated during the development process of a 
sufficiently complex engineering product can be stored and managed directly within the CAD model by using annotation-based strategies, which contribute to better model perception and facilitate alteration and reusability for the creation of new products."

The main objectives can be summarized as:

1. Examine parametric model complexity in terms of design methodology, modeling practices, reusability, and communication of design intent.

2. Examine the role of CAD model annotations as a mechanism to store, transfer, and communicate design intent information.

3. Evaluate the strengths and limitations of existing annotation tools available in modern $\mathrm{CAD}$ environments.

4. Evaluate the overall efficiency and assess the effects of annotations on design reusability and model alteration activities.

5. Determine the relationship between annotated 3D CAD models and user perception in terms of understanding of design intent.

6. Evaluate the role of model annotations on the communication of design intent information.

7. Define the architecture of a software system that implements annotation-based tools and manages design intent information in an explicit manner.

\section{Research Scope}

CAD modeling is a broad research topic that involves a variety of sub-topics such as model representation, data exchange, geometry optimization, modeling processes, assemblies, etc. Similarly, how to represent design knowledge effectively is a fundamental issue in knowledge management, and representation models and formats can vary greatly as they support different design activities. In both cases, this research has been constrained to three specific domains.

First, the intention of this research is not to provide a new theoretical framework for knowledge-based management, nor is it to develop a system to capture and process all types of design knowledge. There are already sophisticated mechanisms (commercial and academic) for managing design 
knowledge. In many cases, however, they do not find acceptance in industry because of the complexity of the tools, the additional user workload involved in maintaining complex information, and the lack of incentives for the user. The focus of this research is geometric design intent information and the practical use of annotation-based mechanisms as carriers of design intent information. This information is intended to help designers understand design modeling know-how, and also facilitate model reuse. The goal is to provide a simple system that allows designers to add and retrieve relevant information to and from a 3D model while maintaining a robust repository of design information regarding the evolution of design changes. The proposed system is based on enhancing some of the existing functionalities available in commercial CAD systems. As a result, a modular solution that can be seamlessly integrated within the CAD environment is provided, so users can incorporate design information to CAD models from an already familiar interface. In this doctoral research, the parametric modeler SolidWorks ${ }^{\circledR}$ and its Application Programming Interface (API) were used, but the proposed solution can be easily implemented in other systems.

Second, in terms of structure (understood as the organization and arrangement of the features and feature interdependencies that comprise the geometry of the CAD model), this research focuses on parametric featurebased solid models, i.e. 3D models created by combining geometric features and defining constraints that establish relations between features. As a result, models are modifiable. Modifications can be made either by changing how the original features were created or by redefining the original constraints. In addition, although most of the results can be easily extrapolated to assemblies and complex multi-body models, the experiments and activities were specifically designed for single parts.

Finally, in terms of information representation, this study focuses on text as a common representation format for design information. Many relevant techniques and algorithms for design information and document management, processing, and analysis rely on textual representations of information. This research takes existing annotation structures as a basis for the development of a more elaborated system. Nevertheless, the proposed architecture is presented as a framework where other types of information elements (hyperlinks, graphical information, external documents, etc.) as well as other engineering and communication tools can be integrated to the CAD environment. 


\section{Contribution to Knowledge}

In an industrial setting, identifying the most appropriate modeling practice for a particular design situation and understanding how the technical details of this best practice can be communicated, stored for future reuse, and shared with other members of the design team are critical factors for success. In this regard, this research is focused on addressing some of the difficulties inherent in traditional model-based engineering systems, parametric design methodologies, CAD model structure, and the current representation and transfer of design information.

The proposed research work uses empirical techniques to evaluate the efficiency and effects of design annotations in 3D CAD models. While several researchers have highlighted the usefulness of CAD model annotations, little data exists on the effectiveness of these strategies. This work is the first study that relates annotation-based mechanisms to alteration tasks, model perception, and CAD model attributes of design reuse. The structures proposed by this research can transform traditional annotations into repositories of design information with the potential to facilitate design management and communication, which could mitigate the impact of impending mass retirements of technical professionals and help organizations cope with a more transient workforce.

As part of this dissertation, an annotation-based software system has been developed. This system provides a framework for the development of engineering and communication tools that are fully integrated with design intent information. In the short term, this type of tool can help automate and optimize specific areas of the product lifecycle. In the long term, the use of more comprehensive annotated models in combination with annotation management tools has the potential to inform numerous procedures related to design documentation and information capture. For example, annotation information can be used as a source for data analysis techniques, which can inspire new semantic technology, automatic methods, and metrics (similar to those used in other disciplines, such as software engineering) to fully assess the quality and reliability of parametric CAD models.

By providing a straightforward mechanism that is fully integrated within the CAD environment, users can be incentivized to annotate 3D models and maintain a repository of design intent information. Functionalities (such as the annotation history proposed in this work), that keep records of annotation information could also motivate and incentivize designers. Just as source 
code comments are useful for software developers, historical annotation information in CAD models can be just as valuable for product designers.

The systematic examination of model complexity and the improved understanding of annotations provided by this study could have significant impacts on design team collaboration and organizational communication. The applicability of annotations as a kind of digital repository can help inform procedures for collaboration and design documentation as well as address how the specific features of particular digital knowledge repositories operate.

Finally, this work has produced a unique and comprehensive set of qualitative and quantitative data, including annotated CAD models, altered CAD models, and attribute data. These data can be used by other researchers attempting to understand design documentation, collaborative design, and communication.

\section{Research Methodology}

This section provides a brief insight of the research approaches considered in this dissertation. The research methodology is based on two stages: research problem definition (literature review) and models and hypothesis validation (experimental validation).

The literature review will examine the main issues surrounding the problem of CAD model reusability (particularly in Model-Based Engineering environments), its connection to the representation and communication of design intent information, and the impediments and current approaches based on annotation structures that are available to assist engineers and product designers in tackling the identified issues. The study within this review of literature will help understand the research gap addressed in this dissertation by focusing on objectives 1 and 2 as set out in section 2 of the this chapter (objectives 3 to 6 will be met through the vehicle of empirical data collection and analysis, while the final objective is derived as a result of the findings from the previous objectives).

During the literature review stage, special attention will be paid to existing annotation mechanisms, its feasibility to represent design information, and the challenges involved in implementing these solutions.

For the majority of this dissertation, an experimental research methodology was used. A number of comparative experimental studies were designed to 
collect information that is qualitative in nature. This information usually involves user performance in the form of time required to complete a series of CAD manipulation tasks. Complete descriptions of the experimental and comparative studies and the analysis methodologies are elaborated in Chapters V and VI.

All experiments presented in this document were conducted in an academic setting with a group of engineering students. Data were collected in simulated design scenarios such as in situations where inadequate modeling assumptions can be made by designers; or when design decisions need to be made and multiple options are available. The results of these studies are used to lay the foundation for a more comprehensive type of annotation (extended annotation) and for the implementation of a software prototype that is tested and validated in subsequent chapters.

\section{Dissertation Outline}

This dissertation is divided into six chapters.

\section{Chapter I: Introduction.}

This chapter provides an overview of the rationale and purpose of this research. It is here where the context and scope are explained and the research gaps are identified. Details regarding outcomes and new knowledge contributed by this work are provided.

\section{Chapter II: Literature Review}

This chapter provides an analysis of previous relevant studies, an identification of research gaps, and how these gaps are address by this work. The following areas are reviewed: New Product Development Process, Model-Based Engineering, Standards Supporting the Model based Enterprise, Product data quality context, Model Complexity and Reusability, and Design Intent Communication.

\section{Chapter III: Parametric CAD Modeling Practices}

Review of parametric modeling techniques and comparison of formal modeling strategies. Analysis and visualization of feature interdependencies and their relation to model complexity and reusability are discussed.

Chapter IV: Communication using Design Annotations 
This chapter provides an overview of annotation mechanisms for engineering design and presents the results of a series of experimental studies aimed at measuring user performance and model quality in two design annotation scenarios. First, in situations where inadequate modeling assumptions can be made by designers; and second, when design decisions need to be made and multiple options are available. Annotation implementation challenges inferred from the results of these experiments are also discussed.

\section{Chapter V: Extended Design Annotations}

A description of the proposed method and structures is provided. Validation results and the architecture of related annotation-based communication and integration tools are presented.

Chapter VI: Integration in Collaborative Environments

Further exploration of the extended annotation model and description of three practical applications: integration with PLM systems, annotation history module, and video conferencing module.

Chapter VII: Discussion, Conclusion, and Future Work

Summary of results and findings from the research study. Conclusions, limitations, and future research directions are also discussed. 


\section{Chapter II Literature Review}

\section{Introduction}

In this chapter, previous work from various areas of relevance to this research is presented. Emphasis is placed on annotation mechanisms. First, a section on Model-Based Engineering provides an overview of this relatively new product development paradigm to define the specific context where this research fits. Second, a review of CAD model alteration and design reusability in the context of product quality provides a theoretical framework for this study and puts the problem of reusability in perspective. Third, an examination of design intent communication and annotation technologies specifically addresses the importance and practical challenges of knowledge representation in the context of a model-based environment as well as the limitations of existing mechanisms to incorporate product information to CAD models. Finally, the state of the art of CAD communication is examined in terms of its relationship to annotations, other communication tools, and integration with information systems.

\section{Model-Based Engineering (MBE)}

With the advent of new computer technologies, flexible data formats, and more sophisticated engineering tools, it is now possible to perform many engineering tasks using digital models. The Model-Based Engineering (MBE) paradigm is an approach to product development that uses CAD 
models rather than documents as the data source for all engineering activities throughout the product life cycle. The core idea is that models can be used to drive all aspects of the product lifecycle and data is created once and reused by all downstream stakeholders [Lubell et al., 2012]. This means that all the required engineering information is communicated to everyone from one source, the digital model.

[Frechette, 2011] understands digital models as the most appropriate vehicle for the delivery of product information. Any number of 3D views of the model can be defined and annotated for specific operations, such as manufacturing planning, production simulation, and materials procurement. Additional views can be specifically annotated for other operations, such as quality assurance and inspection.

Model-Based Engineering is founded on the concept of Model-Based Definition (MBD). Authors [Quintana et al., 2010] describe a product's MBD as a dataset comprising the model's precise 3D geometry and annotations. The annotations specify manufacturing and life cycle support data, known as Product Manufacturing Information (PMI), which may include Geometric Dimensions and Tolerances (GD\&T), material specifications, component lists, process specifications, and inspection requirements. The dataset constitutes a complete definition of the product, without the need for additional documents, such as 2D drawings. Traditional $2 \mathrm{D}$ drawings are not needed when annotations are directly linked to geometric elements in the model, and are properly arranged so they can be viewed without interfering with the model.

Because the MBD is so inherently rich in information, particularly in the case of large assemblies, it is necessary to implement management systems that can efficiently and securely track, control, and manage product data. Typically, this is done by database tools such as Product Lifecycle Management (PLM) systems capable of complete dataset tracking. Therefore, while CAD tools are certainly important, there are other components required to complete the MBE infrastructure, as shown in Figure 1.

An organization that implements MBE is referred to as a Model-Based Enterprise. [Whittenburg, 2012] defines Model-Based Enterprise as a fully integrated and collaborative environment founded on 3D product definition detail and shared across the enterprise with the intent to enable rapid, seamless, and affordable deployment of products from concept to disposal. The same author also states that the key component in this approach is the 
product definition, or $\mathrm{MBD}$, which is described as a 3D model and its associated data elements that fully describe the product definition in a manner that can be used effectively by all downstream customers in place of a traditional drawing. In this context, 3D CAD models serve as the central element from which all engineering processes and outputs flow (analysis results, design decisions, bill of materials, etc). They become the source for delivering documentation and not just a means for creating $2 \mathrm{D}$ drawings.

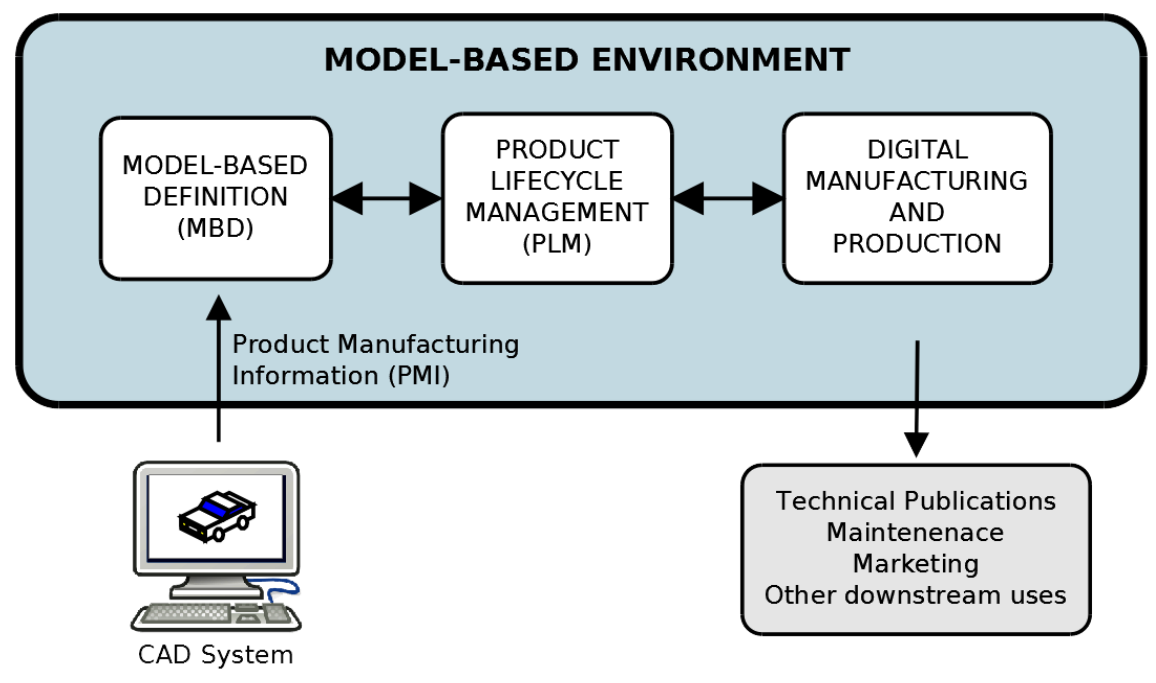

Figure 1. Basic MBE Infrastructure

MBD offers important benefits to manufacturing companies and their customers. In a recent study conducted by the Aberdeen Group, significant time and cost savings were identified when model-based techniques were compared to conventional practices [Aberdeen, 2006]. Another study found time savings of a factor of three for first-article product development and a factor of four for engineering change management [Boehm et al., 2010]. In a different study, [Quintana et al., 2012] quantified the gains of administering the engineering change order process in a MBD context. They conducted a case study in an aerospace company, where reductions of about $11 \%$ in the average processing time and cost were achieved.

Recent developments such as the implementation of product manufacturing information modules in CAD systems and the availability of new formats for viewing and exchanging models now make MBE possible and affordable 
Annotation Mechanisms to Manage Design Knowledge in Complex Parametric Models and their Effects on Alteration and Reusability

even for small manufacturers. To assist in the transition, the DoD Engineering Drawing Modeling Working Group (DEDMWG) offers the MBE index to assist organizations in setting specific goals to achieve the desired capabilities. The MBE levels defined by this group are shown in Figure 2.

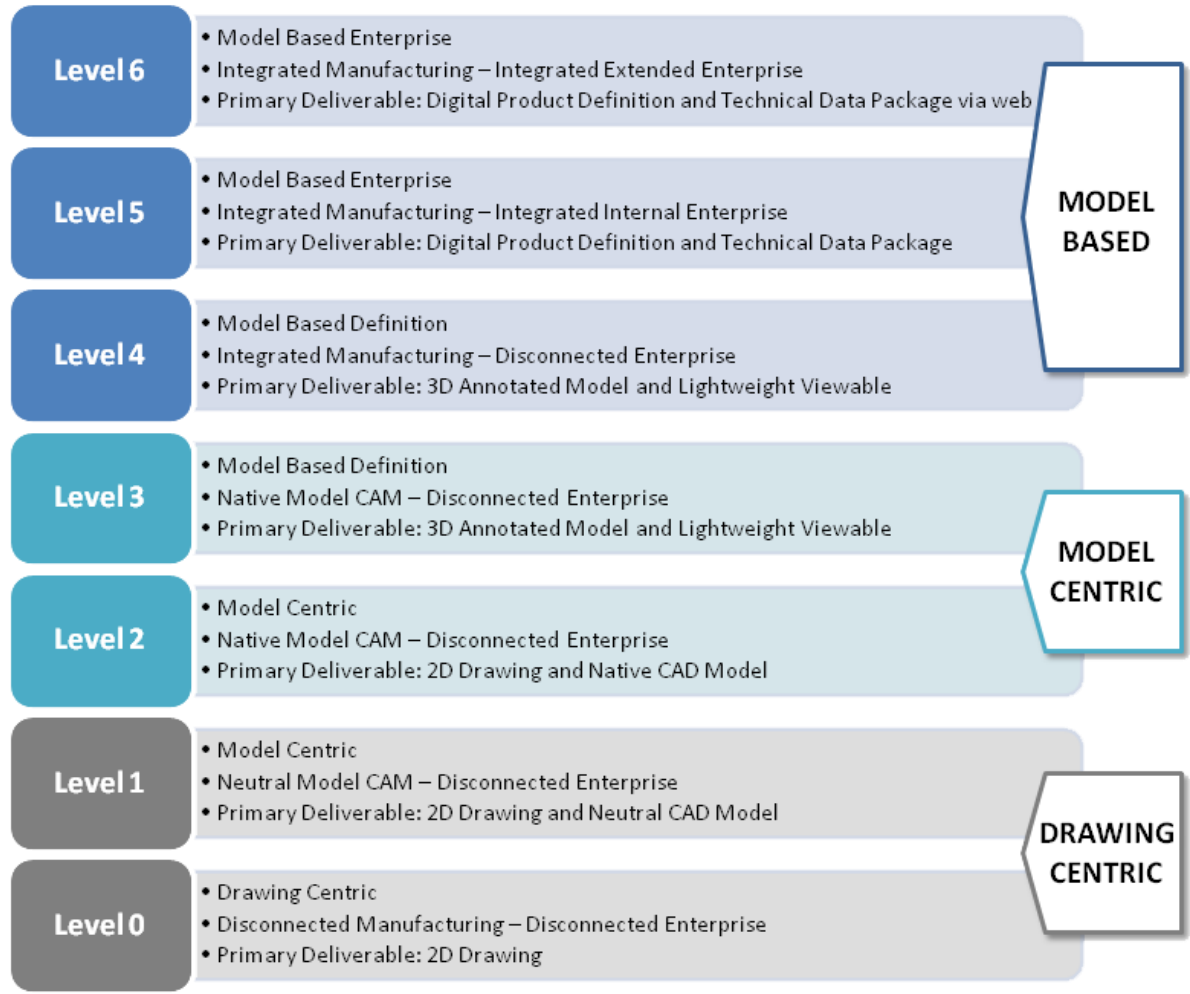

Figure 2. MBE Levels defined by DOD Engineering Drawing Modeling Working Group. Adapted from [Lubell et al., 2012]

The top critical issues affecting the adoption of the MBE were identified, discussed, and reported by [Frechette \& Huang, 2010]

- Requirements and standards for completely annotated product models

- Standards to define requirements for product manufacturing information 
- Long term product data retention requirements

- Technical data quality validation processes

In this regard, the report released by the International Council on Systems Engineering (INCOSE) [INCOSE, 2007] proposes a strategy to migrate from the traditional document-centric approach to a model-based approach that can be fully integrated into existing engineering processes. This initiative identified the development of standards as a critical area for achieving the 2020 Vision.

Similarly, the vision of [FIATECH, 2010], an international collaborative effort of associations, government agencies, and industry, working together to accelerate the adoption of innovative practices and the deployment of emerging and new technologies in the capital projects industry, is of a highly automated environment integrated across all phases of the lifecycle, where information is available on demand to all interested stakeholders. Manufacturers have also identified the need for an MBE infrastructure as a crucial factor for cutting costs and improving competitiveness, and recognized the development and validation of standards as fundamental components for success [Frechette, 2011].

The significance of the MBE paradigm can be observed in a number of initiatives. The most relevant examples include the official non-profit group for MBE [MBE, 2014] and the efforts of the National Institute of Standards and Technology (NIST) Engineering Laboratory, an agency of the US Department of Commerce, which has been actively involved in the development of MBE standards for a number of years. This organization, along with the US Office of the Secretary of Defense (OSD), host an annual Model-based Enterprise and Technical Data Package Summit at NIST [Lubell et al., 2012] to raise visibility on common challenges of the MBE paradigm in both the public and private sectors. Participants from industry and government meet to share the latest technological developments and best practices for model-based engineering, and to continue work on the MILSTD-31000 Technical Data Package (TDP) standard [MIL, 2013] to include requirements for 3D models. The Department of Defense (DoD) Engineering Drawing and Modeling Working Group is responsible for updating MILSTD-31000 to support delivery of model-based technical data for defense systems.

Despite the number of studies that have been conducted that support the MBE paradigm, comparative case studies and more conclusive evidence are 
needed to determine and measure the tangible benefits of 3D models over traditional 2D drawings when equivalent information is included in them. Furthermore, the exact procedures, associated implementation costs, and practical steps that can lead companies to these savings have not been formally established, although the technology and infrastructure to support MBE exist. Nevertheless, the adoption of MBE practices has become a reality in industry, as shown by the increasing number of companies and organizations that are transitioning to model-based paperless environments [Lubell et al., 2012].

This research focuses on the efficient communication of design information within MBE environments. A practical model-based scenario is assumed to be already in place, without making any references or comparisons to traditional 2D drawings.

\subsection{Standards supporting Model-Based Engineering}

In order for information to be robust and communication to be effective, consistency is required in the creation and presentation of the digital model. Maintaining the integrity of product information is the responsibility of all users and anyone who may add or change the model during its creation and revision.

Standards are fundamental building blocks in engineering as they establish consistent protocols that can be universally understood and adopted. Standards are usually formal documents that define uniform engineering or technical criteria to simplify product development and ensure reliability, compatibility, and interoperability.

In the context of the MBE, standards are crucial as they dictate rules and guidelines for an efficient implementation and performance. Standards have formalized many aspects of the way product information is presented, interpreted, and exchanged in a model-based environment, and supported the adoption of the MBE by many organizations and manufacturing companies.

There are five areas that group MBE standards over the product lifecycle, according to [Herron, 2013] and as shown in Table 1 and Figure 3. While most standards were initially focused on mechanical parts, the intent is for them to provide a foundation for use in any discipline. The aspects of interest to this research are shown in red. 
Table 1. MBE relevant standards [Herron, 2013]. The standards of interest to this research are shown in red.

\begin{tabular}{|ll|}
\multicolumn{1}{c}{ Standard } & \multicolumn{1}{c|}{ Description } \\
\hline ISO 10303-242 & Content and format data exchange using STEPS and PLCS \\
\hline ISO 14306: 2012 & Content and format data exchange using JT with Parasolid \\
\hline ISO 14739-1 & Content and format data exchange using 3D PDF \\
\hline ASME Y14.41 & Digital Product Definition Data Practices \\
\hline ISO 16792:2006 & Digital Product Definition Data Practices \\
\hline ASME Y14.100 & Engineering Drawing Practices \\
\hline MIL-STD-31000A & Standard practice for Technical Data Packages \\
\hline NAS 9300-007 & $\begin{array}{l}\text { Long Term Archival and Retrieval (LOTAR) of technical } \\
\text { product documentation }\end{array}$ \\
\hline S1000D & $\begin{array}{l}\text { International specification for Technical Publications using } \\
\text { a common source database }\end{array}$ \\
\hline
\end{tabular}

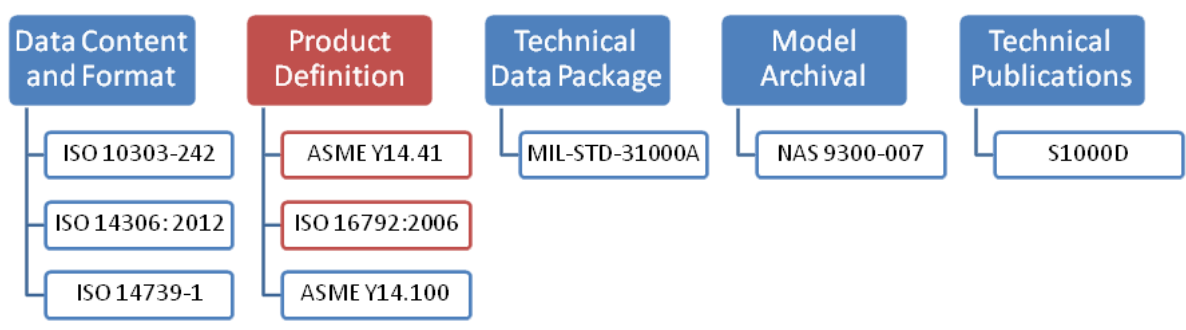

Figure 3. MBE relevant standards by area [Herron, 2013]. The standards of interest to this research are shown in red.

The standard ASME Y14.41 (Digital Product Definition Data Practices) was developed by the American Society of Mechanical Engineers (ASME) in collaboration with experts from industry and academia in 2003 to apply existing requirements developed for two-dimensional drawings equally to the output from three-dimensional models. A revision of this standard was published in 2012 when the content was reorganized to improve the arrangement of different topics in the text [ASME, 2012]. Content related to "Saved Views" was moved to a different standard, ASME Y14.3, and information on unequally or unilaterally disposed profile tolerances was moved to ASME Y14.5-2009. 
Annotation Mechanisms to Manage Design Knowledge in Complex Parametric Models and their Effects on Alteration and Reusability

ASME Y14.41 has many similarities and overlapping requirements with the standard MIL-STD-31000A [MIL, 2013]. However, MIL-STD-31000A defines the entirety of the technical data package required to deliver modelonly products to the US government.

The international standard ISO 16792:2006 was developed by the International Organization for Standardization (ISO) based on ASME Y14.41-2003 guidelines [ISO, 2006]. Using ISO 16792 as a reference, a more specific standard, the "SASIG 3D Annotated Model Standard" was developed in 2008 for the automotive industry, through the Strategic Automotive product data Standards Industry Group (SASIG) [SASIG, 2008]. This SASIG standard complements ISO 16792:2006 by providing rules to document all areas (i.e., design, manufacturing, service) of the product development process in the context of vehicle design and manufacturing. This standard is of particular interest to this research, as will be discussed in future chapters.

Both ASME Y14.41 and ISO 16792 set guidelines for the logical association of product information to geometric elements with the objective of making annotated models comprehensive and reusable, as seen in Figure 4.
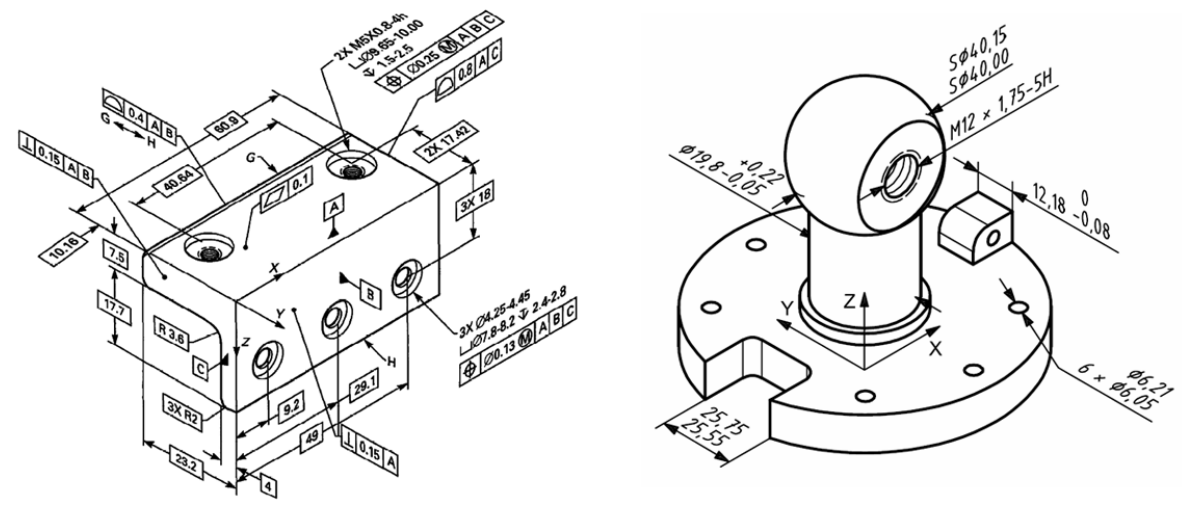

Figure 4. Annotated models from ASME 14.41 (left) and ISO 16792 (right)

In an attempt to mitigate the transition from 2D drawings to 3D models, the standards support two methods of product definition: model-only, and model and drawing in digital format, and distinguish between annotations (defined as "dimension, tolerance, note, text, or symbol visible without any manual or external manipulation") and attributes (defined as "dimension, tolerance, 
note, text, or symbol required to complete the product definition or model feature of the product that is not visible but available upon querying the model"). The focus of this research is exclusively on product information linked to models.

The goal of product definition standards is to make annotated models comprehensive by providing specific guidelines to distribute dimensions and tolerances on orthogonal planes. This mechanism mimics the dimensioning rules for 2D drawings in a 3D environment. However, 3D models can easily become cluttered, as will be discussed extensively in future chapters, even with a small number of annotations and a carefully arranged layout. It becomes evident that practical management of annotations is a critical issue to provide an effective and efficient implementation of the model based definition approach.

Although neither ASME nor ISO standards provide explicit information about using and managing free textual annotations (which this research intends to use to express design intent), the SASIG standard recommends the use of groups, layers, or links to views or sections of the geometry to make the model readable. As this standard states, "turning on all annotations in a complex model may make viewing the annotation and/or model very difficult" [SASIG, 2008]. Because we anticipate a large volume of information included in the annotations when used to deliver design intent (especially when working with complex models), the previous considerations are essential from a usability point of view for a successful implementation of our proposed annotation model.

\subsection{Product and Manufacturing Information in CAD Systems}

The ability to attach information in the form of annotations to 3D models has been supported by major CAD packages for many years. Despite its early use by designers, most tools were proprietary and software-dependent. Information was difficult or impossible to share with other systems. The lack of a common set of rules and guidelines to regulate annotation practices created inconsistencies in formats and methods, which has had an impact in the use and adoption in production environments. This situation has changed with the release of product definition standards.

In commercial CAD packages, practical implementation of product definition standards is currently available using Product and Manufacturing Information (PMI) modules. PMI tools allow users to convey product definition information such as Geometric Dimensioning and Tolerancing 
Annotation Mechanisms to Manage Design Knowledge in Complex Parametric Models and their Effects on Alteration and Reusability

(GD\&T), 3D textual annotations, surface finish and material specifications directly from the CAD environment.

There are many CAD software providers and products for different engineering fields. Among them, the market of CAD systems is dominated by four vendors Dassault Systèmes (DS), Autodesk, PTC and Siemens. As summarized in Table 2, high-end CAD systems (CATIA, NX and Creo) provide PMI modules with full support for product definition standards. Medium systems show different levels of implementation. Neither ASME Y14.41 nor ISO 16792 are supported by the 2015 version of Autodesk Inventor, although the software does provide a proprietary implementation of annotations via the "engineer's notebook."

Table 2. Support of standards in commercial PMI modules

\begin{tabular}{|ccccc|}
\hline Vendor & CAD Software & ASME & ISO & $\begin{array}{c}\text { PMI support in } \\
\text { APIs }\end{array}$ \\
\hline DS & CATIA 3DExperience R2014x & Y14.41 & ISO 16792 & Yes \\
\hline Siemens & NX® ${ }^{89}$ & Y14.41 & ISO 16792 & Yes \\
\hline PTC & Creo ${ }^{\circledR}$ 3.0 & Y14.41 & ISO 16792 & Yes \\
\hline DS & Solidworks ${ }^{\circledR}$ 2014 & Y14.41 & ISO 16792 & Yes \\
\hline Siemens & Solid Edge ${ }^{\circledR}$ ST7 & Y14.41 & --- & Yes \\
\hline Autodesk & Inventor ${ }^{\circledR 2015}$ & --- & --- & Yes \\
\hline
\end{tabular}

PMI modules offer functionality such as manipulation of annotations, definition and selection of annotation planes, visibility control, meta-data management, and tools for creating standard dimensioning and tolerancing annotations. In addition, all CAD applications reported in Table 2 allow access to annotation information via their corresponding Application Programming Interfaces (API), which will be used extensively during the implementation stage of the annotation model. Further details are provided in future chapters.

Just like 2D drawings have done for a number of years, most PMI tools and processes available in modern CAD systems are designed to simply display product information in 3D models for human consumption [Lubell et al., 2011]. Visualizing and understanding the data is ultimately the user's responsibility. However, recent advances in manufacturing technologies allow direct processing of product information data, regardless how the information is displayed graphically. For instance, PMI may be used by 
some Computer-Aided Manufacturing (CAM) software for Numerical Control (NC) machine tool programming or tolerance analysis [Frechette, 2011]. As a result, PMI is becoming more semantic and able to carry increasingly elaborated data structures. In fact, part of this research is based on extending the current functionalities of PMI tools.

\section{CAD Quality and Reusability}

One of the most important contributions of modern CAD to accelerate the product development process is the ability to reuse and make alterations to existing models in an efficient and relatively easy manner. According to the linguistic model proposed by [Contero et al., 2002], three levels of CAD quality can be distinguished, the third of which, the semantic/pragmatic level, considers the capability of the CAD model for reuse and modification. Making changes or reusing a particular CAD model may be simple, difficult, or impossible, depending on the semantics associated to the modeling procedure chosen by the original creator. In this regard, according to many authors [Iyer et al., 2005; Jackson \& Buxton, 2007; Ullman, 2010], an important success factor in new engineering design and development methodologies is the ability to apply knowledge obtained in previous design processes to new designs, which directly translates to CAD model reusability.

Design reuse is not a new problem. More than 30 years ago, [Gunn, 1982] estimated that approximately $20 \%$ of the parts initially thought to require new designs actually needed them; $40 \%$ could be built from an existing design and 40\% could be created by modifying an existing design. In 1998, [Anderl \& Mendgen, 1998], in relation to the creation of real life complex CAD models, stated the following: "If it is difficult to create a model then it is even more difficult to reuse it for variation of modification purposes." More recently, [Iyer et al., 2005], citing [Ullman, 2010], reported that the majority of design problems found in industry involve the application of previous knowledge and the redesign of existing products. Today, the high pressure put on enterprises for optimizing and accelerating product development processes is giving design reuse a crucial role in industrial environments.

There is evidence of the necessity of effective knowledge management methods for establishing efficient design and reutilization processes. [Bodein et al., 2013] reported that reusability of existing models was a critical factor 
Annotation Mechanisms to Manage Design Knowledge in Complex Parametric Models and their Effects on Alteration and Reusability

when determining $\mathrm{CAD}$ modeling strategies in the automotive industry. According to an industry study by the Aberdeen Group [Jackson \& Buxton, 2007], significant time and cost savings were reported in cases of companies reusing design elements. Furthermore, all engineering organizations surveyed in the study report the reuse of existing designs at some level, but the top performers intentionally dedicate resources and deploy methods and technologies to capitalize on reusability. There are, however, important problems that need to be overcome to effectively implement design reuse. As described by the same study [Jackson \& Buxton, 2007], the obstacles related to $\mathrm{CAD}$ model reuse are the same obstacles that largely impede design reuse. These challenges as well as the procedures that companies are currently following to mitigate them are shown in Table 3.

Table 3. Challenges and responses to CAD model reusability (adapted from [Jackson \& Buxton, 2007])

\begin{tabular}{|c|c|c|}
\hline & CHALLENGE & PROCEDURE \\
\hline 1. & $\begin{array}{l}\text { Model modification requires expert } \\
\text { CAD knowledge }\end{array}$ & Train users to increase CAD skills \\
\hline 2. & $\begin{array}{l}\text { Models are inflexible and fail after } \\
\text { changes }\end{array}$ & $\begin{array}{l}\text { Design for wide range of } \\
\text { modifications }\end{array}$ \\
\hline 3. & Users cannot find models to reuse & $\begin{array}{l}\text { Centralize design data in library } \\
\text { accessible structure }\end{array}$ \\
\hline 4. & $\begin{array}{l}\text { Only original designer can change } \\
\text { models successfully }\end{array}$ & Detail design information in model \\
\hline
\end{tabular}

The relationship between challenges 1 and 2 is clear, as they are both involved in the creation of better designed models. CAD users need to be skilled in CAD methods, tools, and technology in order to develop models that are reusable and easy to maintain. Challenge 3 can be categorized as a data management problem that requires effective tools and information management mechanisms such as Product Lifecycle Management systems (PLM) to be in place. Finally, challenge 4 demands formal methods to integrate various types of design information (GD\&T, material specifications, manufacturing instructions, etc.) within the CAD model. Access to design information has been a problem for many years, as reported by the 1994 survey, which estimated that designers spend about $60 \%$ of their time searching for the right information [Leizerowicz et al., 1996].

According to the classification of CAD modeling errors proposed by Yan \& Han [Yang \& Han, 2006], three primary types can be identified. The first 
type is related to topological and geometric inaccuracies, such as discontinuities, small and void faces, and self-intersection problems. Currently, these problems are well covered by standards such as VDA 4955/4.1 [VDA, 2006] and SASIG PDQ 2.1 [SASIG, 2005]. These problems can be termed as intrinsic problems (associated to morphological quality, according to [Contero et al., 2002]). The second type of problems is related to the product data exchange process, i.e. when models are converted to and from different formats. These errors are usually due to mismatches in the numerical inaccuracies of the geometric kernels upon which CAD systems are built. It is hypothesize that this second type of problems may also be linked to the syntactical quality suggested by [Contero et al. 2002]. These two types of problems, which can be termed extrinsic, are out of the scope of this study.

However, there is a third group of problems that are caused by users as a result of poor or incorrect practices with the CAD system (i.e. semantic quality [Contero et al. 2002]). According to [Yang \& Han, 2006], a third to a half of all quality problems arise either from poor design skills or from the inexperience of designers. In this case, two complimentary strategies can be applied. First, according to [Mandorli \& Otto, 2013], current education should provide more strategic knowledge and understanding to enable students to use CAD systems as knowledge-intensive design and communication tools to properly develop and convey design intent. Second, model annotations carrying hints, warnings, and indications to specific modeling questions can be useful to help users understand the reasons behind complex modeling decisions. Additionally, making this information available outside the model easily allows the use of external information systems to manage and analyze the design knowledge contained within the extended annotations. A new contribution to the second strategy is proposed as a contribution of this work.

\section{CAD Modeling Strategies for Reusability}

The first step to guarantee model reusability is the selection of an efficient modeling methodology. Although many companies require designers to follow specific modeling methods defined by internal CAD guidelines (sometimes dictated by previous experiences and/or senior designers' expertise), there is a limited amount of published information about effective modeling procedures. In professional environments, efficient design methodologies and best practices may lead to important competitive 
advantages. For this reason, this type of information is often protected, unless it is released in the form of patents [Landers \& Khurana, 2004].

In a history-based parametric CAD system, three-dimensional features are added to the model in an associative relationship (parent/child) with the feature(s) they are immediately connected to, which results in a tree-like structure where every node represents a feature and the design intent of the model is implicitly represented by these relations. This tree structure is commonly known as design tree, feature tree, or history tree. By leveraging the model's design tree, designers can make changes that automatically propagate to other related child features. To do so, however, they must ensure that the model will react to changes in a predictable manner by anticipating and defining proper geometry relations and dependencies [Bodein et al., 2013].

Parent/child interdependencies between features are the root of most regeneration problems in parametric modeling. Ideally, these interdependencies must line up with the design intent of the part so changes can be performed in an efficient manner by propagating automatically from parent nodes to child nodes. When these interdependencies are not defined properly, designers are often forced to rebuild the model to some degree to re-establish new design intent [Salehi \& McMahon, 2009].

In the area of history-based parametric design, the selection of an efficient modeling procedure largely determines the degree of flexibility and reusability of the final model; the more efficient the methodology, the more flexible and adaptable the model. Furthermore, an inefficient modeling strategy may cause the model to become unstable, even when minor alterations are performed, which often requires a complete rebuild of the part from scratch [Salehi \& McMahon, 2009]. To minimize the time and effort involved in modifying existing models, it is necessary to select an appropriate modeling methodology based on the characteristics and requirements of the part. In this context, a number of studies have attempted to determine the factors that allow designers to define the modeling procedure that is more suitable for the specific geometry of the model [Hartman, 2005].

The three major modeling strategies that have been published and tested are Delphi's horizontal modeling [Landers \& Khurana, 2004], explicit reference modeling [Bodein et al., 2014], and resilient modeling [Gebhard, 2013]. 


\subsection{Delphi's Horizontal Modeling}

Horizontal Modeling was invented and originally implemented by Delphi Technologies, Inc, one of the world's leading automotive parts manufacturers. The methodology was patented by [Landers \& Khurana, 2004] as a method to create higher quality models by eliminating the need to recreate or repair CAD data throughout design cycles. According to these authors, many of the limitations of traditional feature-based modeling are related to the vertical nature of its modeling structures, i.e. the inherent parent/child relations defined between features. The goal of the horizontal modeling methodology is to define a manageable structure that ensures that any changes made to a feature of the model do not affect subsequent features. Structures that lead to unstable models are avoided by eliminating the parent/child relations established between nodes in the model's design tree.

In this methodology, all features reference base datum planes or offset datum planes, as opposed to other features, ensuring that there are no direct dependencies between features (see Figure 5). These reference planes serve as parent nodes for groups of features, which are defined at the same level (horizontally) in the design tree. As a result, the risks of unstable models and unwanted effects caused by altering or deleting a feature are minimized.

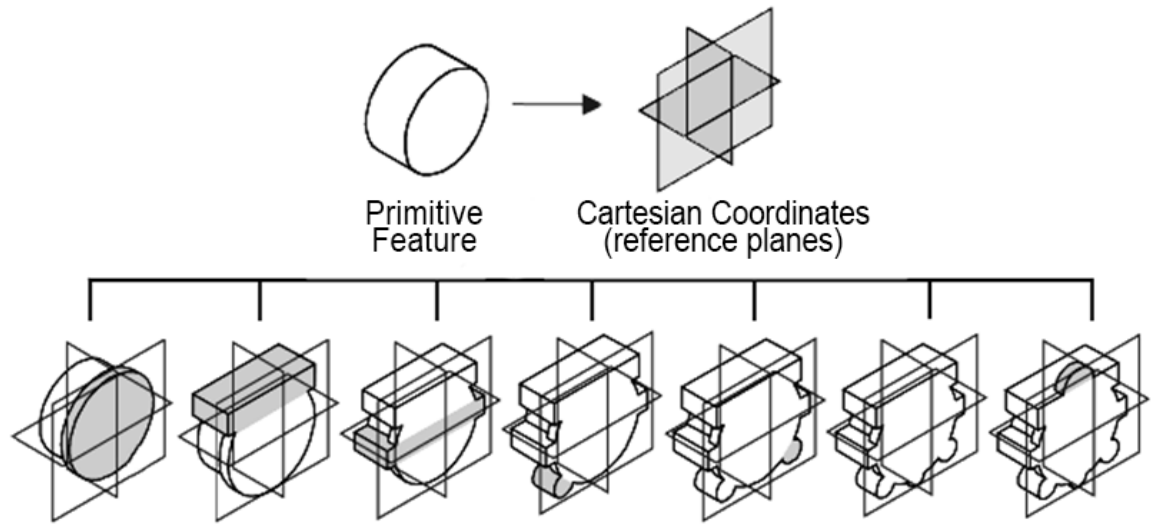

Figure 5. Horizontal modeling strategy, adapted from [Landers \& Khurana, 2004]

In general, the design tree of a 3D model created with a horizontal methodology is simple, flat (most features are located at the same level in the tree), and easy to understand; the chain of feature dependencies is usually 
short, which makes the sequence of modeling steps easy to trace. Nevertheless, many authors agree that dependencies between features are important and extremely valuable when used properly. Parent/child dependencies are essential elements in parametric systems that enable designers to create truly flexible and adaptable models. Although it is useful to employ reference planes in certain situations, ignoring the benefits of sharing and relating features to existing geometry would be taking a step backwards in the creation of reusable models and the incorporation of design intent within the model's feature tree. Additionally, the fact that horizontal modeling is a patented methodology means that designers are technically not allowed to use it without a patent license from Delphi Technologies, Inc.

\subsection{Explicit Reference Modeling}

Parametric CAD systems implement various modeling operations to allow designers to build three-dimensional geometry. Some of these operations require the definition of two-dimensional sketches; others must always be linked to existing geometry. A general classification of modeling operations and all possible constraints that can be associated to the current shape was provided by [Bodein et al., 2014] based on previous research from [Betting $\&$ Shah, 2005] (see Figure 6).

From this classification, the authors proposed a modeling methodology based on the explicit management of functional references with the aim of minimizing the creation of constraints linked to the existing geometry. Although constraints using new geometry can be defined when creating $2 \mathrm{D}$ sketches on planar surfaces or on individual sketch elements, most design features can be used as support elements for new constraints. The authors indicated that the use of existing geometry as support for constraints is only required in localized modification operations (see Figure 6). In most cases, existing geometry to support constraints can be replaced by external reference elements such as planes.

In the explicit reference modeling methodology [Bodein et al., 2014], parametric constraints are divided in two categories: category I and category II. The first category includes constraints that can be defined by elements that are not part of the geometry of the model. Category II includes constraints that must always reference existing features or certain aspects of the geometry.

For constraints in category II, direct references to the model's geometry must be replaced by explicit references to external elements such as points, 
planes, or lines (see Figure 7). The goal is to build a design tree that is simple and easy to understand, and has a small number of parent/child dependencies [Wang \& Nnaji, 2005]. The strategy is to place dependent features (child nodes) as close to the parent as possible while paying special attention to features that are likely to be altered of eliminated. By placing child features close to their corresponding parent, the structure of the design tree becomes natural and easy to follow, even for users that are not familiar with the model or have not participated in its creation. Models created by following a logical sequence of steps are better understood by designers than those created with unstructured strategies [Johnson \& Diwakaran, 2011]. 


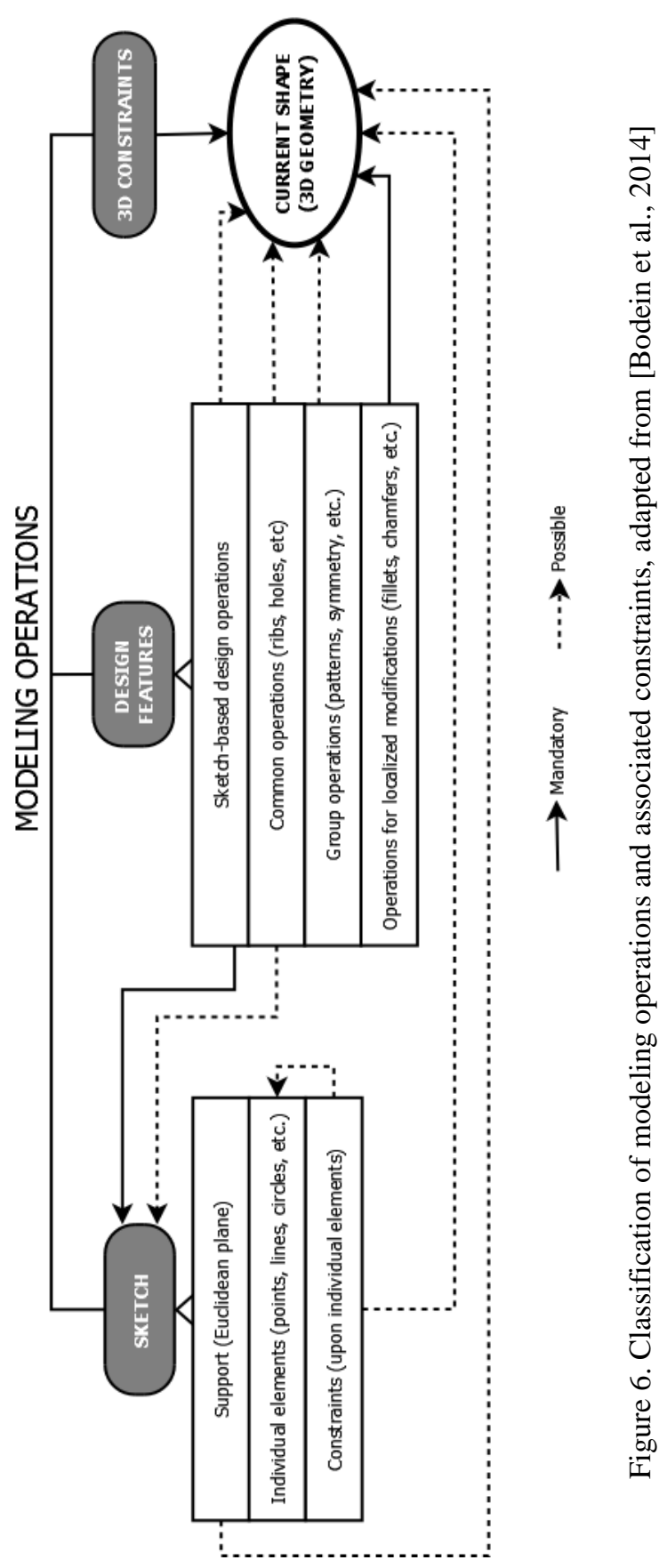




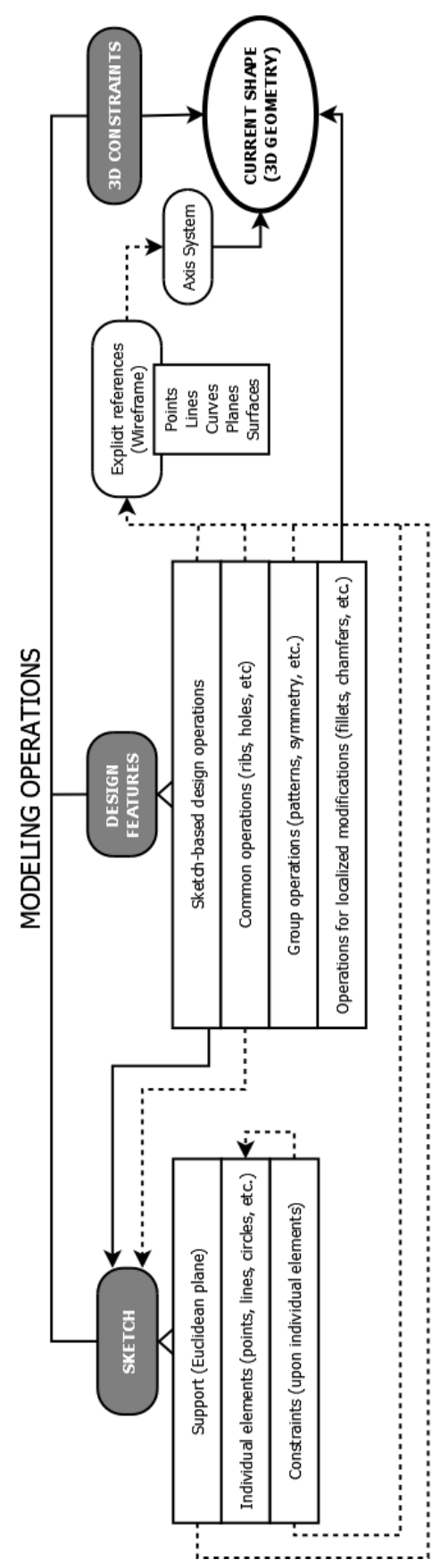

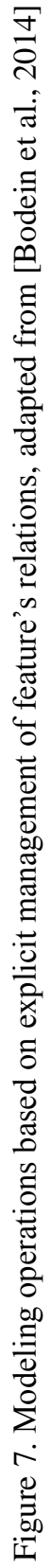


The fact that parent/child relations can still be used in this methodology means that model inconsistencies may still occur. In complex parts with a significant number of features, a chain of parent/child relations may become problematic when a feature located at a high level in the tree hierarchy (a parent of multiple child nodes) is altered or removed. When this happens, all children (and grandchildren, etc.) are affected. To minimize the unwanted effects in these situations, it is recommended that all features that are likely to be modified or removed from the model are placed at the lowest levels in the tree structure.

\subsection{Resilient Modeling Strategy}

Resilient modeling is a methodology built on best practices that was developed by [Gebhard, 2013] with the goal of maximizing the flexibility and robustness of CAD models while minimizing the risk of inconsistencies. It defines a standard format for the design tree, where features are organized in a stable structure, so the model can be changed predictably without spending a great effort understanding the dependencies between features. In this methodology, the design tree is divided into six sections which group the model features according to their function, importance, and volatility. Groups must appear in the correct sequence in the model's design tree. These groups are shown in Table 4.

General reference elements that must be available throughout the entire modeling process are included in the first group. The second group, if needed, contains construction features that will be used as references for subsequent solid bodies. The central features that define the overall shape, orientation, and extension of the 3D model (features that generate material) are included in the Core group (group 3). Major changes in the basic shape of the model will require the modification of features in this group. In general, features included in the first three groups can be used as reference elements for child features.

Specific geometric details that typically remove material from the part such as slots, holes, and threads are included in group 4 as child elements of features from previous groups. Since detailed geometry is likely to be altered or removed, it is recommended that features in group 4 are not used as parent nodes or reference elements for subsequent features, unless absolutely necessary. 
Group 5, if needed, includes features that add the final geometric elements to the model and do not require further child features, such as patterns, drafts, and mirrored elements. Finally, cosmetic and finishing features such as fillets and chamfers are always added last to the design tree and included in a separate group (group 6).

Table 4. Feature groups defined by the Resilient Modeling Strategy [Gebhard, 2013]

\begin{tabular}{|c|c|c|c|c|}
\hline Group & Description & Typical Features & Notes & Links \\
\hline 1- Ref & $\begin{array}{l}\text { All "Reference" entities } \\
\text { are first, making them } \\
\text { available/visible to all } \\
\text { features }\end{array}$ & $\begin{array}{l}\text { Ref Bodies, } \\
\text { Layouts, Sketches } \\
\text { Ref Planes, Coord. } \\
\text { Sys, Images }\end{array}$ & $\begin{array}{l}\text { No } \\
\text { Solids }\end{array}$ & \multirow{3}{*}{$\begin{array}{l}\text { If you can see it in the } \\
\text { background, it is } \\
\text { acceptable to link to it }\end{array}$} \\
\hline 2- Construction & $\begin{array}{l}\text { Construction features such } \\
\text { as Surfaces or 3D Curves } \\
\text { that will be used to define } \\
\text { complex solid features }\end{array}$ & $\begin{array}{l}\text { Surfaces, Project, } \\
\text { Extend } \\
\text { 3D Curves, Trim, } \\
\text { Split }\end{array}$ & $\begin{array}{l}\text { No } \\
\text { Solids }\end{array}$ & \\
\hline 3- Core & $\begin{array}{l}\text { A "Super Based Feature" } \\
\text { that determines the } \\
\text { model's shape, extents, } \\
\text { and orientation }\end{array}$ & $\begin{array}{l}\text { Extrude, Sweep, } \\
\text { Thin Wall } \\
\text { Revolve, Loft, Shell }\end{array}$ & $\begin{array}{l}\text { Add } \\
\text { Material }\end{array}$ & \\
\hline 4- Detail & $\begin{array}{l}\text { Detail features complete } \\
\text { the shape by only linking } \\
\text { to the Core group }\end{array}$ & $\begin{array}{l}\text { Extrude, Sweep, } \\
\text { Hole } \\
\text { Revolve, Loft, } \\
\text { Thread }\end{array}$ & $\begin{array}{l}\text { Remove } \\
\text { Material }\end{array}$ & $\begin{array}{l}\text { Links to other groups } \\
\text { are acceptable, except } \\
\text { within the "Detail" } \\
\text { group }\end{array}$ \\
\hline 5-Modify & $\begin{array}{l}\text { Tilt faces and replicate } \\
\text { features then add any } \\
\text { "Final Features" }\end{array}$ & $\begin{array}{l}\text { Draft, Pattern } \\
\text { Mirror, Final } \\
\text { Features }\end{array}$ & & \multirow{2}{*}{$\begin{array}{l}\text { If you can see it in the } \\
\text { background, it is } \\
\text { acceptable to link to it }\end{array}$} \\
\hline 6- Quarantine & $\begin{array}{l}\text { Volatile features that } \\
\text { should not be parents }\end{array}$ & $\begin{array}{l}\text { Chamfer, Blend, } \\
\text { Round }\end{array}$ & $\begin{array}{l}\text { Largest } \\
\text { first }\end{array}$ & \\
\hline
\end{tabular}

In addition, specific guidelines are provided to name files and features in the design tree so it is easier to identify them when alterations need to be made. According to this methodology, features must be named based on its design intent and its functional purpose and characteristics (see Figure 8). This simplifies editing tasks by providing a clear and intuitive mechanism to recognize and follow the sequence of operations that was performed throughout the modeling process. 


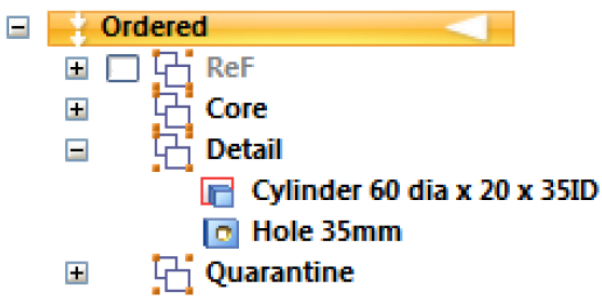

Figure 8. Sample design tree with proper RMS naming [Gebhard, 2013]

The goal of this strategy is to create a stable model using a simple and intuitive structure so minimum effort is required to comprehend its design intent. This structure also allows designers to detect building errors in the model and easily identify the problematic areas and their sources. To ensure that models are created according to the methodology, RMS also provides a checklist with the key items designers must verify when creating the geometry.

In the following chapter, a comparative analysis of these modeling strategies will be presented. Some aspects considered include the rationale to avoid the creation of unnecessary feature interdependencies, the sequence and selection criteria for those features, and the effects of parent/child relations on model alterations. The general internal structure of the models created according to each methodology will be analyzed and compared in terms of their robustness and flexibility when the geometry is modified.

\section{Data, Information, and Knowledge}

To stress the scope of this doctoral work, it is important to review the differences between three terms: data, information, and knowledge. These terms are often mistakenly used interchangeably in many contexts, but their differences are significant and cannot be ignored.

According to [Harding, 1996], the term "data" refers to raw words or numbers, the meaning of which is dependent upon the context in which it is used. Data are simply symbols with no context and no relationships. For instance, the number 2 , without any context, is a single piece of data.

Data that has been processed, organized, and/or structured in some way so that it has a certain meaning within a given context so as to make it useful is called "information." [Harding, 1996]. For instance, "2 miles from point A to point B" has meaning if you are driving your car from point A to point B. 
The concept of "knowledge" is much more difficult to define as it can have a variety of answers. In fact, different authors have studied knowledge and provided different definitions, interpretations, and classifications. According to [Harding, 1996], knowledge is information with details regarding how this information may be used or applied. Knowledge is not directly available but is obtained by interpretation of information deduced from analysis of information [Chandrasegaran et al., 2013]. Knowledge can be classified according to different criteria and along several dimensions. Knowledge management, however, is out of the scope of this dissertation.

Similarly to the definition, the understanding of knowledge within the realm of engineering design varies depending on the context. This doctoral research focuses on the specific type of design information involved in the process of creating and altering a CAD model. Although this information is commonly known as design intent, which includes the reasons and rationale behind geometric modeling decisions, it could sometimes be considered a type of knowledge, depending on what the designers is trying to communicate and how that information is represented.

\section{Design Intent Communication}

The second factor that determines model reusability involves design intent communication. To achieve the full benefit provided by 3D CAD models, users that interact with them should understand how and why the component was created and designed in a specific way; namely, they must understand its design intent [Henderson, 1993].

In this work, "design intent" or "geometric modeling intent" will be used distinctly to express the reasons that motivate a designer to perform some specific CAD modeling actions. It also expresses the manner in which the designer expects the geometric model to behave when it is modified [Alducin-Quintero et al., 2012]. Design intent becomes critical in situations where the user altering a model is not the original creator, such as in collaborative design scenarios. Questions related to design intent are often asked when engineers attempt to interact with CAD models created by others [Karsenty, 1996]. Explicit communication of design intent is especially valuable for the reutilization of complex 3D CAD models, in which important amounts of modeling time are invested.

It is hard to find a precise definition of design intent, since the term can be interpreted in slightly different ways [Conklin \& Yakemovic, 1991; 
Brissaud, 2003; Horvath \& Rudas, 2003]. After an exhaustive literature review to identify common elements, authors [Iyer \& Mills, 2006] proposed their own definition, which is generally widely accepted: "Design intent contained in legacy CAD is the insight into the design variables (design objectives, constraints, alternatives, evolution, guidelines, manufacturing instructions and standards) implicit in the structural, semantic and practical relationships between the geometric, material, dimensional and textual entities present in the CAD representation." [Iyer \& Mills, 2006].The importance of design intent and the advantages of an explicit representation were summarized by [Pena-Mora et al., 1993]:

- Changes in complex projects require certain design decisions to be modified during the development process. When the justifications defined during the initial stages are lost, they need to be recreated, which has a negative impact on project costs and development times. The ability to store, process, and retrieve this information can significantly improve productivity.

- When design intent information is represented explicitly and is easily available for review, the overall quality of the product increases.

- Explicit representation of design intent leads to a more intelligent use of resources and knowledge.

- Efficient communication of design intent is essential for integrating solutions and transferring design knowledge.

To benefit from the functionalities provided by modern CAD systems to quickly and efficiently modify existing designs, users interacting with the model must understand the reasons behind the modeling process. In other words, the design intent of the model must be appropriately captured and understood by the person making the changes [Karsenty, 1996; Rodriguez et al., 1998; Regli et al., 2000].

Given that one of the initial "promises" of CAD was the ability to store and easily alter existing models [Liker et al., 1992], the fact that models are often remodeled from scratch is somewhat of a "paradox" [Mandorli \& Otto, 2013]. Despite major advances in CAD technology, models are difficult, or impossible, to alter when design intent information is lost or not communicated properly [Salehi \& McMahon, 2009]. 
Some considerations to communicate design intent effectively include how this type of information can be captured, represented, managed, processed, and stored. These are active areas of research [Iyer \& Mills, 2006] and still poorly addressed by current industrial tools [Bracewell, 2009]. Although some success has been reported using semi-automated tools [Ganeshan, 1994; Quereshi 1997; Myers, 2000; Bracewell et al., 2009], the task of capturing design intent cannot be completely automated [Shum et al., 2006], and thus requires designers to be actively involved throughout the entire process. Unfortunately, it has been shown that designers are often reluctant to spend time adding additional information to their models [Szykman et al., 2001]. Therefore, any tools to support interaction with design intent information must be easy to use, intuitive, and integrated with existing solutions [Chandrasegaran et al., 2013].

Design knowledge is a broad term that can be understood at different abstraction levels throughout the design process. The diversity and complexity of knowledge involved in engineering design makes it difficult to capture and represent this information. The complex and highly dynamic nature of knowledge management has led to the development of various types of tools for various applications: knowledge sharing, expert systems, knowledge retrieval and query, etc. On an abstract level, for example, representation and manipulation of the model's function is a crucial issue during conceptual design, as indicated by [Umeda et al., 1996]. Because current CAD systems do not support functional design, the authors implemented a software tool called Function-Behavior-State (FBS) Modeler, which supports functional design during both the analytical and synthetic phases of conceptual design [Umeda et al., 1996]. According to [Gero, 1990] a representation framework with sufficient expressive power to capture the nature of the concepts is required for design. He proposed a knowledge representation schema based on design prototypes to separate knowledge from computational processes. The use of this representation provides a translation between design syntax and semantics [Gero, 1990]. Other examples of knowledge capture systems include the Market Driven Design System [Harding et al., 2001], used to capture and collect market information within a product model using fuzzy inference.

How to represent design knowledge effectively is a fundamental issue in knowledge management, and representation models and formats can vary greatly as they support different design activities. Despite the variety of methodologies and systems available, there are still barriers in terms of practical implementations in industrial environments that need to be 
overcome: information confidentiality, lack of adequate training in the use of knowledge management, language, affordability, cost, technology levels, etc. [DuPlessis, 2008].

The most sophisticated approaches often involve the use of complex external systems, such as Compendium [Shum et al., 2006] and DR editor (DRed) [Bracewell et al., 2009] (both based on the concept of Issue based Information System or IBIS [Kunz \& Rittel, 1970]), and rely heavily on human intervention, especially for interpreting and entering information into the system. Other approaches include argumentation-based models such as Decision Representation Language (DRL) [Moran \& Carroll, 1996], which was further extended by Software Engineering Using Design RATionale (SEURAT) [Burge, 2008], and the Question, Option and Criteria (QOC) technique which emphasizes discussions of alternatives regarding artifact features [Moran \& Carroll, 1996].

Most representation models are related to specific domains. For example, functional representations focus on describing how the device works [Chandrasekaran et al., 1993]. A Rationale Construction Framework (RCF) was also suggested to capture rationale information by monitoring designers' interaction with a CAD system [Myers et al., 2000]. Although progress has been made, the most advanced techniques and algorithms for data mining and design document processing rely heavily on textual representation of design knowledge [Liang et al., 2012].

This work specifically focuses on geometric design intent information, i.e. the type of knowledge directly related to the CAD model's geometry information (explanations of why a CAD model is modeled the way it is or why certain modeling steps have been performed). In this context, the goal is to provide a simple yet efficient mechanism that can be fully integrated within the $\mathrm{CAD}$ environment and allows designers to add relevant geometric information to a $3 \mathrm{D}$ model efficiently while maintaining a repository of design intent information to help designers understand design modeling know-how, and also facilitate the reuse of models.

\section{Annotations in Engineering Design}

There are few effective tools available to communicate information in support of collaborative efforts [Fuh \& Li, 2005; Shen et al., 2008]. The use of $3 \mathrm{D}$ annotations to communicate design information is an active area of research. It has also become more popular in industry, especially since the 
appearance of MBE standards and their subsequent integration in major CAD packages.

With the high demands of industry, researchers began to study the suitability of 3D annotations to carry design information [Boujut \& Dugdale, 2006; Davies \& McMahon, 2006]. The knowledge captured and shared in CAD models is not merely helpful for design; it represents a major source of value for an organization. This knowledge includes the CAD modeling process and its implicit design intent. Explicit knowledge (engineering formulae, technical documents, etc) does not capture all necessary knowledge. The reasons and rationale behind modeling decisions also need to be available [Lee \& Yang, 2000]. Favorable results have been reported in terms of time savings when users are required to make alterations to annotated models as opposed to non-annotated ones [Alducin-Quintero et al., 2011].

In general terms, an annotation can be defined as a piece of information, normally in textual form (although they can also adopt other forms such as images, audio, hyperlinks, etc), attached to an existing information object at a particular location [Ovsiannikov et al., 1999]. A generic annotation contains two elements: the annotation content and the annotation anchor. The annotation content is the data of the additional information piece. The annotation anchor is the pointer referencing the location at which the annotation is placed [Brush et al., 2001].

The purpose of an annotation is to provide notes, comments, explanations, clarifications, descriptions, or interpretations about the object being annotated. In a product development context, annotations are typically used to enhance engineering models with information that is valuable, but difficult to communicate otherwise, such as dimensions, tolerances, or manufacturing information. In a $3 \mathrm{D} \mathrm{CAD}$ modeling environment, annotations are referred to as model-based annotations (or simply model annotations) to emphasize the three-dimensional nature of the element, as opposed to purely two-dimensional drawing-based annotations.

Model annotations are usually represented as blocks of text anchored to a specific aspect of the 3D model via leader lines [Ding et al., 2009]. Although most commercial CAD packages provide annotation tools, only a few allow an active interaction with the information. Annotations are typically used as pointers, whose purpose is to draw the attention of the designers to a specific area of the model. The different types of annotations according to current 
Annotation Mechanisms to Manage Design Knowledge in Complex Parametric Models and their Effects on Alteration and Reusability

model-based standards and thus available in commercial PMI modules are shown in Table 5.

Table 5. Annotations in Model-Based standards

\begin{tabular}{|ll|}
\hline Annotation & Description \\
\hline Envelope dimensions & 3 overall boundary dimensions of the part. \\
\hline Block tolerances & $\begin{array}{l}\text { May be a note that defines all default tolerances to be applied } \\
\text { to the product unless otherwise specified. }\end{array}$ \\
\hline Material requirements & $\begin{array}{l}\text { Annotation text shall source from centrally controlled } \\
\text { material library. }\end{array}$ \\
\hline Finish requirements & $\begin{array}{l}\text { May show in notes or displayed as an annotation related to a } \\
\text { particular feature surface. }\end{array}$ \\
\hline Title block information & $\begin{array}{l}\text { Number, description, drawn and approved by, revision date, } \\
\text { etc. }\end{array}$ \\
\hline $\begin{array}{l}\text { Non-block tolerance } \\
\text { dimensions }\end{array}$ & $\begin{array}{l}\text { Dimensions shown in an annotation that override model } \\
\text { geometry queries. Most commonly used to describe holes. }\end{array}$ \\
\hline Full dimensions & Defines full product definition. \\
\hline Site map & Index of available views. \\
\hline Full notes & Defines full product definition. \\
\hline Auxiliary views & $\begin{array}{l}\text { Provides convenient views to view all required product } \\
\text { definition. }\end{array}$ \\
\hline
\end{tabular}

In the context of engineering design, the importance of annotations in the context of the design process was summarized by [Boujut \& Dugdale, 2006] in the form of three points:

a. Annotations are considered containers for meaning, playing an important role in the cognitive synchronization between designers.

b. Annotations are intermediary elements that play an interface role, mediating the interactions between designers.

c. Annotations have the ability to capture the intent behind design decisions.

The authors specifically acknowledge the significance of the last point, stating that it is "of prime importance in design research today" but "very poorly addressed by current industrial tools." Likewise, authors [Bracewell \& Wallace, 2003] identified a need for specific tools to help designers capture the design intent of their decisions. 
The use of model annotations as a tool to supplement CAD models by including product data and design information within the geometry has been the subject of active research [Davies \& McMahon, 2006; Ding et al., 2009b; Alducin-Quintero et al., 2012]. Authors [Patel et al., 2008] recognized the need to enhance the geometric model of a product with information that is relevant to the various stages of the product lifecycle (such as design rationale, context, extra information needed for a certain point of view, etc) as critical for CAD engineering model representations. This suggests a natural transition from model-based annotations to model-based design annotations by transforming regular model annotations into carriers of design knowledge. Annotation representation unification was identified by [Li et al., 2009] as a major challenge of current product lifecycle management (PLM) systems in order to be universally recognizable across different domains, platforms, and systems. Various annotation architectures and software prototypes have also been developed [Boujut \& Dugdale, 2006; Sandberg \& Näsström, 2007; Ding et al., 2009b]. Examples of the application of semantic technology in the engineering design process are also abundant. For example, [Szykman et al., 2000] suggested the use of a functional taxonomy to aim the management of knowledge into product design repositories. [Au \& Yuen, 2000] proposed a linguistic approach to create sculptured models, and showed taxonomic relations between three levels of extractions at object level, feature level and geometry level. [Fu et al., 2003] attempted to extract features from a data exchange product model using a taxonomy, which defines relationships between design features and manufacturing features for feature identification in CAD models.

There are, however, a number of challenges to using 3D annotations to carry design knowledge. Some of these challenges are related to the technology, such as defining efficient internal structures and data types for the annotations or developing an interface or system to enter and retrieve the information [Ding et al., 2009]. Other challenges involve user interaction, such as the representation of the annotation content (what needs to be included in the annotation and in what form, so the information is communicated effectively) or the visualization of heavily annotated models (the problem of visual clutter and annotation overload).

\subsection{Classification of Annotations}

Annotations can be classified in a variety of ways. Authors [Ding et al., 2009] suggested six major categories of annotations based on audience, 
targeted media, rendering system, usage and function, representation, and storage location. This classification is illustrated in Figure 9.

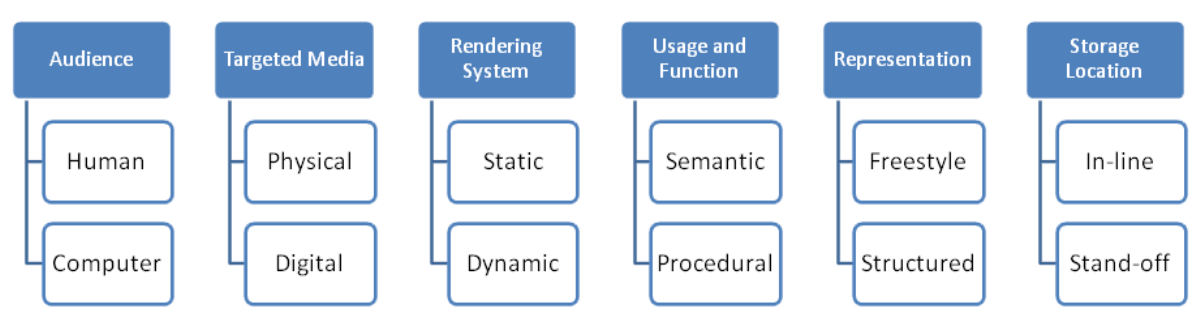

Figure 9. Classification of annotations [Ding et al., 2009]

\subsubsection{Audience}

Annotations can be directed at a human audience or to a computer audience. In the first case, annotations are created, consumed, and shared by individuals or teams. For example, an evaluation team (annotator) may add notes as feedback for a second team (audience). In the case of a computer audience, annotations are directly fed to computer software that manipulates the information, e.g. searching, filtering, data mining, etc. In order to be processed by computer programs, annotation structures must be strictly formalized by complying with a specific syntax or schema [Davies, 2008].

\subsubsection{Targeted Media}

Annotation can be targeted to a wide variety of physical media or digital media. Annotations are often made on paper documents (books, exams, etc), and also on digital files (text documents, multimedia and video files, etc). In the case of 3D objects, annotations can be applied to a geometric mock-up in physical form or a CAD model in digital form.

\subsubsection{Rendering System}

In terms of rendering systems, digital annotations can be classified as static and dynamic. The static approach implies that annotations are hardcoded with the content being annotated and delivered as saved. Dynamic annotations are adaptable, and its delivery depends on the specific request. For example, in a web-based annotation system, annotation data can be merged with the original document as a new webpage or saved separately. In 
the latter case, annotation data and the associated object can be rendered in a desired representation style as requested [Wang, 2005].

\subsubsection{Usage and Function}

Annotations can be classified as semantic and procedural depending on usage and function. Semantic annotations typically describe the information entity and its constituents. The primary purpose is to clarify and interpret a subject within a certain context to avoid confusion with the meaning in other domains. Procedural annotations describe the procedures or processes of manipulating the information and its constituents [Kiryakov et al, 2004]. In other words, semantic annotations are descriptive (about remembering and clarifying), whereas procedural annotations involve thinking and sharing.

\subsubsection{Representation}

Annotations may be classified as freestyle (informal) or structured (formal). In freestyle annotations, information is created and added to the target without a formal structure or syntax. Structured annotations follow a predefined schema (described in a certain language such as XML), and are managed in a structured way. Freestyle annotations are easy to create and provide freedom to the user. However, structured annotations are easier to maintain, can be efficiently processed by software, and allow self-annotation functionalities [Wang, 2005].

\subsubsection{Storage Location}

Annotations can be classified as in-line and stand-off in terms of how data is stored. In-line (or internal) annotations require storing the annotation information internally within the model, whereas a stand-off (or external) approach involves maintaining the annotation information in a separate repository outside the model. The advantages and disadvantages of both methods are listed in Table 6 , which is a modified version of the table prepared by [Ding et al., 2009].

In general, authors agree that stand-off annotations provide greater benefits than in-line for use with CAD models [Patel et al., 2008; Li et al., 2009], particularly when the data needs to be shared. The independence and flexibility of stand-off annotations allow a progressive expansion of the metadata (if required) without changing the geometric representation of the model, as well as a multi-layered structure so multiple annotation files can be used to provide different views or levels of annotations for different users or purposes [Patel et al., 2008]. 

their Effects on Alteration and Reusability

Table 6. Advantages and disadvantages of mark-up strategies (*new items added to the original list created by [Ding et al., 2009])

\begin{tabular}{|c|c|c|}
\hline STRATEGY & ADVANTAGES & DISADVANTAGES \\
\hline In-line & $\begin{array}{l}\text { - } \quad \text { Easy implementation } \\
\text { - } \quad \text { Wide applications } \\
\text { * Full integration with the } \\
\text { model (low maintenance) } \\
\text { - } \text { Efficiency in terms of } \\
\text { processing and manipulation } \\
\text { - } \text { Already supported by most } \\
\text { CAD systems }\end{array}$ & $\begin{array}{l}\text { - Original document changed } \\
\text { - Difficulty for multiple } \\
\text { independent sets of markup } \\
\text { * Difficult to share } \\
\text { information in collaborative } \\
\text { environments }\end{array}$ \\
\hline Stand-off & $\begin{array}{l}\text { - } \begin{array}{l}\text { Non-change of representation } \\
\text { method used for the original } \\
\text { object }\end{array} \\
\text { - Support of multiple } \\
\text { independent sets of markup } \\
\text { - Support of progressively } \\
\text { information update (scalability) } \\
\text { - Capability of re-organization of } \\
\text { information for different } \\
\text { purposes and applications } \\
\text { * Easy distribution of } \\
\text { information in collaborative } \\
\text { environments and over the web. } \\
\text { * Information can be processed } \\
\text { and analyzed separately. }\end{array}$ & 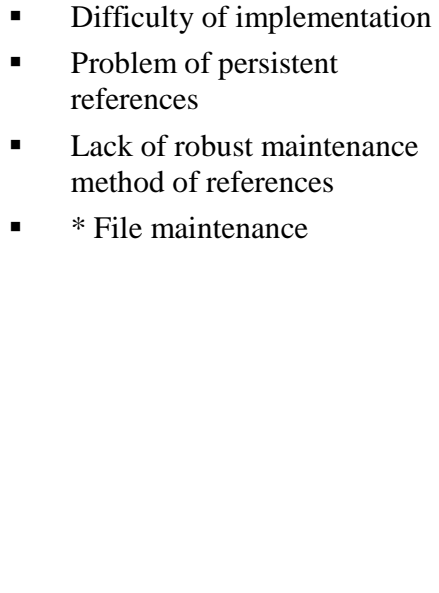 \\
\hline
\end{tabular}

In the context of a PLM system, stand-off annotations are clearly more appropriate considering the global and collaborative nature of the data managed by the PLM. Moreover, the use of eXtensible Markup Language (XML) has been recognized as a common data description standard in current PLM systems [Cheung \& Schaefer, 2009]. Hence, some authors have experimented with this language in order to implement model annotation methods [Ding et al., 2009b; Matthews et al., 2009].

Despite the obvious advantages discussed previously, stand-off annotation strategies in distributed environments are difficult to implement, mainly because of the problem of persistent association of references (also known as persistent naming problem), which is critical in situations where models 
change, such as in product development. To mitigate this problem, some researchers have made attempts to combine both in-line and stand-off annotation techniques. For example, the CAD mark-up environment implemented by [Patel et al., 2009] combines an internal module for the commercial software NX Unigraphics with an external module for 3D PDF viewers. Both environments save annotations to a XML file, which is linked back to a specific part of the CAD model through an external transfer interface.

A similar annotation approach was proposed by [Ding et al., 2008]. In their research, the authors proposed a framework based on lightweight representations of a CAD model and two mark-up environments (internal and external) that accessed multiple XML files, allowing the definition of multiple viewpoints and security levels. A complete interface was implemented to allow designers to enter product information as annotations. As indicated by the authors, the problem of persistent references becomes more critical because of the use of multiple lightweight versions of the model. They also reveal the need to reorganize information for efficient retrieval and maintain annotations during CAD model evolution [Ding et al., 2008].

\subsection{Annotations in Software Development}

An area with an extensive history of design documentation in the form of annotations is computer software. Documentation is an essential aspect of software quality [Kajko-Mattsson, 2001; Van De Vanter, 2002] and a key instrument that developers use to explain what a program does and how code works. It is especially important in large software projects that involve multiple development teams working overtime. The practice of documenting code has been proven particularly effective in code reuse, design communication, and software maintenance (program changes and upgrades) [Haouari et al., 2011], as these tasks are frequently done by software engineers that did not participate in the original developments.

Studies in software engineering have estimated that developers spend as much as $75 \%$ of their time reading and understanding existing code, rather than creating new code [Glass, 2003; Pfleeger \& Atlee, 2009]. This number is a clear indicator of the importance of proper code readability and documentation. Different strategies and methodologies have been developed in software engineering to improve and assess design communication. Software design patterns, source code comments, and software annotations are some examples. 

their Effects on Alteration and Reusability

Software design patterns are used to describe a proven solution to a software design problem with the goal of making the solution reusable. Experimental work has concluded that pattern-relevant maintenance tasks were completed faster or with fewer errors if redundant design pattern information was provided [Prechelt et al., 2002]. Other authors have shown that graphically documented design patterns can achieve significantly better performance than those that were provided with source code alone [Gravino et al., 2011]. Research has confirmed that maintenance effort is significantly reduced when design pattern instances are properly documented and provided to the programmers [Scanniello, 2010].

Source code comments are pieces of information written in natural language and embedded in the source code (see Table 7) used for a variety of purposes, including conveying information about program structure and semantics, as well as personal thoughts, explanations, reminders [Storey et al., 2009], and notes for future changes [Haouari et al., 2011]. Software annotations [Kellens et al., 2010] attach additional metadata to various entities (classes, methods, etc.). Some modern programming languages (e.g., Net's attributes or Java) support annotations. These annotations can communicate a programmer's intent, or it can be used with other specific software engineering tools [Kellens et al., 2010].

Table 7. Source code documentation example (from Wikipedia)

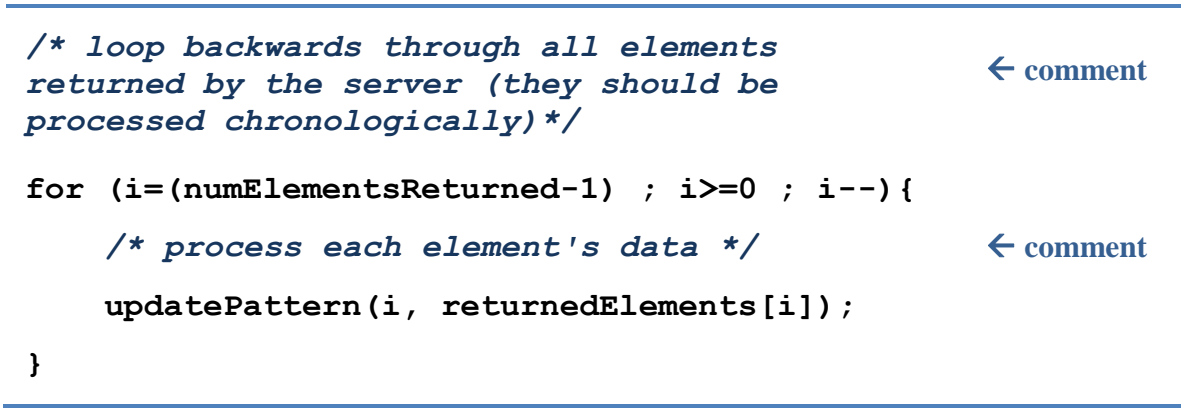

Previous research reveals that source code comments are the primary resource used by developers to understand code [Souza et al., 2006]. In fact, many program failures and "bugs" are caused by miscommunication among developers or misinterpretations of code, often triggered by improper or lack of source code documentation [Tan et al., 2007]. Other failures are caused by 
mismatches between code and comments, which usually happen when comments are not updated accordingly as code evolves [Tan et al., 2007].

Because of its importance in the development process, several scholars in the software engineering field have focused their efforts on improving code readability [Elshoff \& Marcotty, 1982], extracting information from comments with the purpose of determining what makes an annotation useful [Khamis et al., 2010] and developing techniques to automatically annotate source code [Buse \& Weimer, 2010]. In addition, metrics for software quality analysis have been proposed to assess the value of source code comments and annotations [Khamis et al., 2010; Schreck et al., 2007].

In the CAD community, researchers and practitioners have begun to experiment with the potential of 3D CAD annotation tools as a means to add design notes to 3D models [Alducin-Quintero et al., 2012]. Despite the progress made by the software engineering industry, few studies address the quality assessment of 3D annotations in CAD models and the inclusion of Product and Manufacturing Information as a native part of a CAD file. There are significant similarities between "software design intent" in the software engineering field and "design intent" in the product development and engineering design process. Many of the existing techniques for improving code reuse and design communication in the software domain can potentially inspire new strategies for improving CAD model reuse and product design communication. This work will examine CAD annotations using analogous tools to those used in the evaluation of software code.

\subsection{State of the Art in 3D Annotation}

In terms of annotation of 3D models, several approaches and software systems have been suggested. [Davies, 2008] developed a hybrid annotation framework for both semantic and procedural annotations that allows multiple viewpoint annotations. For example, a model annotated with manufacturing and analysis viewpoints may be useful for a manufacturing engineer, who can determine the hole type as a counter-bored feature that requires drilling, and an analyst with no interest in manufacturing information, who may find that the hole is a fixing hole (implying some boundary condition for the analysis).

Another semantic approach based on viewpoint was proposed by [Thouvenin et al., 2005; Aubry et al., 2007]. Their structured system, known as MATRICS (Managing Annotation for Training in an Immersive Collaborative System), maintains annotations into three viewpoints: design 
concept (materials, scientific and technical domains, etc), geometrical description, and methods, and uses an ontology-based knowledge management system to process annotation information.

Another ontology-based hybrid, "Funnotation" [Kitamura et al., 2006], was developed to aid CAM design. Its complete semantic annotation model relies on processing text documents and contains four elements: the function of the device, how the function is achieved, the functional decomposition structure, and alternative solutions to achieve the functions. When the system is filled with sufficient annotation entries, it can automatically suggest suitable parts based on a given functional design specification.

The solution proposed by [Bilasco et al., 2006], the 3D Annotation Framework (3DAF), is an annotation system that stores a set of semantic profiles of 3D scenes. Users can send requests to the annotation repository and retrieve the specific information pointing to corresponding 3D fragments. An integration component translates all fragments and reassembles them into a new $3 \mathrm{D}$ model according to the topology defined by the request.

For architectural applications, a web-based 3D annotation system called the Space Pen Java applet was developed by [Jung et al. 2002]. In this system, users can simultaneously create annotations in 3D models by using specific gestures. Another technology that uses a digital pen in combination with a special paper is ModelCraft [Song et al., 2006]. In this system, a special paper that has been pre-printed with dot patterns can be recognized by a special digital pen. When a digital 3D model is printed, users can use a digital pen to physically create freehand annotations, which can then be transferred back to the digital model.

A summary of the most representative 3D annotation approaches to date is illustrated in Table 8, including some approaches not described above. 
Table 8. Summary of 3D annotation approaches (Target media: 3D models)

\begin{tabular}{|c|c|c|c|c|c|}
\hline Approach & Audience & Representation & $\begin{array}{l}\text { Usage \& } \\
\text { Function }\end{array}$ & $\begin{array}{l}\text { Rendering } \\
\text { System }\end{array}$ & $\begin{array}{l}\text { Storage } \\
\text { Location }\end{array}$ \\
\hline [Davies, 2008] & $\begin{array}{l}\text { Human and } \\
\text { Computer }\end{array}$ & Structured & $\begin{array}{l}\text { Semantic and } \\
\text { Procedural }\end{array}$ & Dynamic & Stand-off \\
\hline $\begin{array}{l}\text { LIMMA } \\
\text { [Ding et al. 2009b] }\end{array}$ & $\begin{array}{l}\text { Human and } \\
\text { Computer }\end{array}$ & $\begin{array}{l}\text { Freestyle and } \\
\text { Structured }\end{array}$ & $\begin{array}{l}\text { Semantic and } \\
\text { Procedural }\end{array}$ & Dynamic & Stand-off \\
\hline $\begin{array}{l}\text { MATRICS } \\
\text { [Thouvenin et al. 2005; } \\
\text { Aubry et al. 2007] }\end{array}$ & $\begin{array}{l}\text { Human and } \\
\text { Computer }\end{array}$ & Freestyle & $\begin{array}{l}\text { Semantic } \\
\text { (Knowledge- } \\
\text { Based) }\end{array}$ & Dynamic & $\begin{array}{c}\text { Not } \\
\text { specified }\end{array}$ \\
\hline $\begin{array}{l}\text { Space Pen } \\
\text { [Jung et al. 2002] }\end{array}$ & Human & Freestyle & Not specified & Not specified & Inline \\
\hline $\begin{array}{l}\text { 3DSEAM } \\
\text { [Bilasco et al. 2005] }\end{array}$ & $\begin{array}{l}\text { Human and } \\
\text { Computer }\end{array}$ & Structured & $\begin{array}{l}\text { Semantic } \\
\text { (Knowledge- } \\
\text { Based) and } \\
\text { Procedural }\end{array}$ & Dynamic & Stand-off \\
\hline $\begin{array}{l}\text { 3DAF } \\
\text { [Bilasco et al. 2006] }\end{array}$ & $\begin{array}{l}\text { Human and } \\
\text { Computer }\end{array}$ & Structured & $\begin{array}{l}\text { Semantic and } \\
\text { Procedural } \\
\text { (Both } \\
\text { Knowledge- } \\
\text { Based) }\end{array}$ & Dynamic & Stand-off \\
\hline $\begin{array}{l}\text { [Pittarello \& Faveri, } \\
\text { 2006] }\end{array}$ & Computer & Structured & $\begin{array}{c}\text { Semantic } \\
\text { (Knowledge- } \\
\text { Based) }\end{array}$ & Static & $\begin{array}{l}\text { Inline and } \\
\text { Stand-off }\end{array}$ \\
\hline
\end{tabular}

\subsection{The Problem of Visual Clutter}

As 3D models become more comprehensive and serve as the central element of the MBE paradigm, both the complexity and number of annotations increase, which can drastically increase the amount of visual information on screen at any given time. This matter naturally demands mechanisms to support the interaction with the information as well as an efficient visualization of the annotations. Displaying all annotations in a complex model quickly creates clutter and confusion, which makes the use of the model impractical. From a usability standpoint, minimizing visual clutter in extensively annotated models is an essential factor to ensure effectiveness in terms of communication of information

The problem of visual clutter has a long history of research, particularly in the areas of cognitive psychology and human factors. However, the term is not easy to define. Intuitively, clutter can be understood as the phenomenon that occurs when one has too many items available at any given time. In fact, many methods related to clutter reduction in human-computer interaction 
involve the elimination of some of those items from the central part of the display [Ahlberg \& Shneiderman, 1994; Fishkin \& Stone, 1995]. Some studies have shown, however, that clutter is not necessarily linked to the number of items, but to a state in which the items cause confusion in the user so they negatively affect performance [Noyes, 1980; Rosenholtz et al., 2005]. Although too much information can certainly cause visual clutter, other factors such as perception (people do not always agree on the level of clutter), user experience, information relevance, and the level of information organization should also be considered [Tufte, 1983].

Management of visual clutter is an important factor in user interface design and information visualization. When too much data (or when data is not well organized) is displayed on a too small area, the value of the information and the visualization as a whole diminishes, affecting usability [Ellis \& Dix, 2007]. Over the years, a vast amount of research has been done in the area of visual search [Palmer, 1994; Wolfe, 1994; Rosenholtz, 2001] and clutter reduction. [Woodruff et al., 1998; Ellis et al., 2005; Ellis \& Dix, 2006]. Many of these techniques rely on the user driving the visualization to less cluttered states [Fishkin \& Stone, 1995]. Other methods such as [Woodruff et al., 1998; Ellis et al., 2005] apply the principle of constant information density to always generate visualizations that are neither too cluttered nor too scattered. A variety of metrics to measure visual clutter have also been proposed [Tufte, 1983; Rosenholtz et al., 2005; Frank \& Timpf, 1994]. Despite the large number of clutter reduction techniques, the diversity of the application domain makes it difficult to find one solution that can be applied to all problems. Furthermore, formal comparative and usability studies are scarce, particularly when compared to the diversity of solutions available [Ellis \& Dix, 2007]

In the domain of annotated 3D models, previous research has focused on algorithms for different annotation styles [Cipriano \& Gleicher, 2008], annotation layouts to prevent occlusion [Stein \& Décoret, 2008], and annotations alignment to automatically arrange annotation information around the model [Ali et al., 2005; Götzelmann et al., 2006]. However, no specific solutions are defined in current CAD annotations standards, and thus, no practical implementations are available in current PMI modules. Reducing clutter is left to the discretion of the user, who often chooses not to benefit from annotation tools because of the additional effort involved in this task. Clearly, there is a need for mechanisms to actively filter and manipulate the annotation information presented on screen (see Figure 10). 


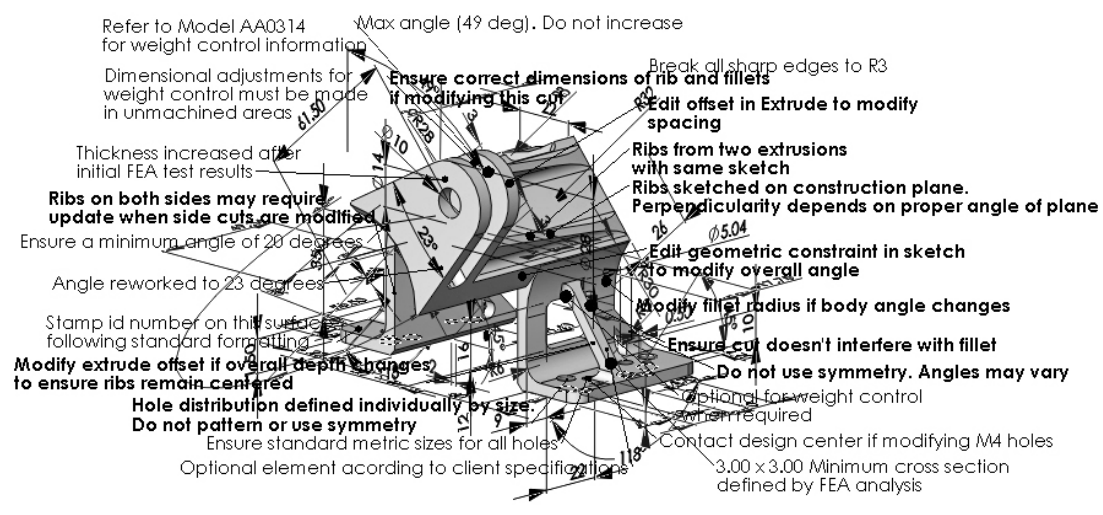

Figure 10. Visual clutter caused by annotations

\section{Collaborative Engineering and Computer Supported Cooperative Work}

Using annotation mechanisms to communicate design information has implications that go beyond 3D modeling. When integrated within a collaborative environment, annotations become part of a larger body of knowledge and design toolsets that make engineering communication more efficient. Part of this research involves incorporating annotation-based mechanisms to other communication tools. For this reason, to understand the specific role of annotations in this context, it is necessary to review recent work in the area of collaborative engineering and Computer Supported Collaborative Work (CSCW).

Modern engineering teams frequently work in an information-technologysupported, collaborative environment, and in virtual teams, where designers, process planners, manufacturers, and even clients communicate and coordinate their projects through information and communication technologies [Chudoba et al., 2005], even when they are geographically dispersed [You \& Chao, 2006].

A distributed product development organization is based on a principle that its designers simultaneously work on their geometric design, Computer Aided Engineering (CAE) or marketing capabilities, all while using the most up-to-date documents and drawings [Abrahamson et al., 2000; Vila et al., 2007]. This model naturally demands a tight interaction and coordination among all participants. Its success relies heavily on a robust information and 
communication infrastructure that can effectively support the "virtual teams" [Londono et al., 1992; Upton \& Mcafee, 1999]. Capturing, processing, and managing all the information that is exchanged during collaboration activities, as well as making this information easily accessible to the appropriate teams, are necessary prerequisites for success [Ahmed, 2005].

Computer supported cooperative work (CSCW) is a broad term that refers to the use of information technology and telecommunications in support of collaborative work [Frivold et al., 1995]. Although there is not a consensus on the exact type of technology that is described by this term, CSCW can be considered an umbrella term that includes communication, shared workspaces, information, content, and group activity support tools [May \& Carter, 2001].

The basic aspects that a CSCW system should demonstrate were summarized by [Tay \& Ming, 2001] in the form of three points:

- Communication among three or more clients.

- Capability for both synchronous and asynchronous interworking.

- Communication content should include each (and any combination) of the following objects: text, audio, video, CAD/CAM files and images.

The previous general aspects are expressed by [May \& Carter, 2001] as a specific set of basic requirements for CSCW systems:

- Functionality: minimum requirements include high-quality audio and video tools, shared 2D whiteboard facilities, and online access to existing project data.

- Data access, management, and security: distributed teams need to share a central database, where single copies of documents are stored and version control is automatically managed. Intelligent data filing, file conversions, and data retrieval are also required.

- Usability, reliability, and support: tools must be simple, easy to use, and as reliable as standard telephone and office applications. Appropriate feedback and multiple platform integration are also expected.

Initial research efforts in the field of collaborative engineering and CSCW have been focused on the development of environments for co-authoring, content and documentation sharing, and message passing [Bly et al., 1993; 
Kao \& Lin, 1998; Rahman et al., 1999]. More recently, the popularization of the Internet has fueled the development of network-enabled and web-based collaborative applications by both academia and industry [Tay \& Roy, 2003; Konduri \& Chandrakasan, 1999; Qiang et al., 2001]. Nevertheless, shared design workspaces are usually closed solutions, which means that integration into existing environments and process chains is difficult and often unreliable [Durstewitz et al., 2002].

The increased trend towards concurrent engineering models and manufacturing globalization has led to the need for sophisticated CSCW tools and collaborative CAD/CAM systems [Kao \& Lin, 1998]. Distributed design environments are no longer based on single standalone CAD workstations, but on a combination of software, facilities, services, and infrastructures [Li et al., 2005] that support collaboration (by increasing responsiveness and information availability, and by enhancing mass customization), communication (by reducing transfer times and data navigation iterations), and content management (by providing consistent and accurate product definition and helpful product viewing) [Toussaint \& Cheng, 2002]. In general, three major types of software applications are recognized by [Fonseca et al., 2006] to be involved in collaborative design:

- Functional Applications: all applications used for content creation and for the development of any product or process, such as $\mathrm{CAD} / \mathrm{CAM}$ packages.

- Management Applications: all applications that administer, control, support, and manage the data created by the functional applications, such as PLM systems.

- Communication Applications: all applications that support and improve communication and interaction among teams, such as email, messaging services, or videoconferencing.

While much research has focused on the challenging area of collaborative creation and modification of CAD models in distributed network environments [Chen et al., 2005], less attention has been paid to communication tools. It is in the context of communication applications, specifically video conferencing, and its connection to annotations, that part of this research is situated. 
Annotation Mechanisms to Manage Design Knowledge in Complex Parametric Models and their Effects on Alteration and Reusability

\subsection{Multimedia Supported Communication Technology for Collaborative Design}

Effective communication and information sharing are key elements of the concurrent engineering model for connecting ideas, requirements, specifications, activities, feedback, and ultimately people [Tay \& Ming, 2001]. The effectiveness and robustness of the communication networks among individuals and teams have a direct influence in many engineering outcomes. In fact, misunderstandings and communication problems are the main cause of bottlenecks and in the current collaborative model [Durstewitz et al., 2002].

The benefits of multimedia and communication tools to support collaborative work have been recognized by various researchers [Greenberg, 1991; Gowan \& Downs, 1994]. Literature confirms that communication tools increase the cooperative awareness of participants in shared environments and play a significant role in the solution of conflicts during the concurrent design process [Fu et al., 2013]. Examples of these tools include: text messages (email, SMS, fax), audio messages (phone and voicemail), two-dimensional and three-dimensional geometry viewers, asynchronous services (file sharing and World Wide Web), and synchronous services (shared applications, whiteboard and video conferencing). Real-time communication and collaboration among geographically distributed teams requires synchronous mechanisms.

Studies in the area of CSCW suggest that video conferencing may be the most valuable communication tool for distributed groups in a collaborative design environment [Gowan \& Downs, 1994]. With the rapid development of network and multimedia technology, video conferencing systems are becoming more and more popular among organizations. Although the effectiveness of video conferencing depends on the quality and responsiveness of the media (bandwidth is a critical factor), and the particular task that the participants are trying to accomplish [Gajewska et al., 1994; May \& Carter, 2001], authors agree on the positive impact of video conferencing in terms of user satisfaction of remote collaborative working [Olson et al., 1995] and successfully resolving tactical tasks collaboratively [Kydd \& Ferry, 1994; Gowan \& Downs, 1994].

Practical uses of video conferencing have been usually tested as a functionality of integrated collaborative environments [Maxfield et al., 1998; Durstewitz et al., 2002; Tay \& Roy, 2003; Fonseca et al., 2006]. In most cases, video conferencing works in combination with a shared design screen, 
where multiple users can visualize the same geometric model at the same time (although only one user can manipulate it at any given time). These shared design environments are often built as separate applications. They are stand-alone tools, disconnected from existing software solutions, which has the following drawbacks:

- A separate application (the shared environment) must be launched and managed independently from the CAD application.

- Integration with existing CAD/PLM systems is difficult, expensive, and often unreliable.

- 3D geometry must be exported or converted to formats such as VRML, XML, or STEPS before it can be used by the application and shared with other users.

- Editing capabilities in collaborative environments are limited (or nonexistent) when compared to the ones offered by popular CAD packages. Therefore, if certain changes have to be made to a model while it is being shared, users are forced to constantly switch between applications.

In this research, collaborative functionalities (video conferencing, shared screen, and connection to model annotations) are added to existing CAD applications and connected to annotation tools, so users can interact and communicate with others directly from their CAD interface without the need to manage separate applications or environments. 


\section{Chapter III Parametric CAD Modeling Practices}

\section{Introduction}

In this chapter, an analysis of history-based parametric CAD modeling is presented. First, the internal structure of parametric CAD models and the implicit representation of design intent are examined. Second, model complexity is studied using an experimental tool specifically implemented for this doctoral research. This tool allows the visualization and measurement of model complexity. Finally, the results of a comparative study of publicly available and professionally accepted CAD modeling strategies and best practices for history-based parametric design are reported: Delphi's horizontal modeling, explicit reference modeling, and resilient modeling.

Some aspects considered in this study include the rationale to avoid the creation of unnecessary feature interdependencies, the sequence and selection criteria for those features, and the effects of parent/child relations on model alterations. An evaluation of these strategies using a group of industrial CAD models is provided. The internal structure of the models is evaluated by comparing their robustness and flexibility when the geometry is modified. 


\section{Approaches to 3D CAD Solid Modeling}

In the domain of $3 \mathrm{D} \mathrm{CAD,} \mathrm{there} \mathrm{are} \mathrm{two} \mathrm{distinctive} \mathrm{approaches} \mathrm{to} \mathrm{solid}$ modeling: parametric (or history-based) and direct (or history-free). A parametric model is a geometric model with predefined parameters that can be changed to create different design variants. Parametric modeling provides a structured approach to $3 \mathrm{D}$ creation that requires users to anticipate certain aspects of the design by defining constraints and relations to ensure that any change will update all related downstream geometry in a predefined way. Direct modeling, on the other hand, provides a method to quickly define and capture geometry. Designers focus on creating geometry rather than building design intent into the models.

Parametric models are based on the design history (the record of modeling operations used to build the geometric shape). In most CAD packages the design history is known as the design tree. At any time during the modeling process, designers can access previous operations in the design tree and change the values that control the geometry to create a variation of the current geometric shape. Direct modeling, however, is history-free. The methodology focuses on geometry rather than features, making it suitable for situations where speed and flexibility are essential.

Both modeling approaches have advantages and drawbacks. In this section, a comparative evaluation of both methods is provided. The conclusions drawn from this study are used to justify the focus of this doctoral research on feature-based parametric solid modeling.

\subsection{Parametric Modeling vs. Direct Modeling}

History-based parametric CAD technology is currently a mature and commonly deployed technology that offers sophisticated functionality to create geometry. However, this method also has some serious drawbacks, particularly when working with complex models.

Some of the advantages and disadvantages of parametric modeling technology are the listed below.

Advantages:

- Mature technology.

- Excellent for design optimization and reusability.

- Powerful approach for products that are highly engineered 
- Many designers with experience in one or more of these tools.

- Growing direct editing capabilities for improved flexibility.

Disadvantages:

- Data exchange (Design history is typically lost in translation from one CAD format into another).

- Must plan ahead and follow good modeling practices.

- Lack of transparency for the user. Interfaces are not WYSIWYG (What You See Is What You Get).

- Large and complex models require a large design history, which translates into a large file size and potential slow performance.

Direct Modeling systems provide tools for quickly creating and modifying 3D models directly with no attention to the modeling process. They allow a direct manipulation of elements of a geometric model in 3D space. Direct modeling is a lightweight and flexible approach ideal to make radical part and assembly changes synchronously and in context. Some of the advantages and disadvantages of direct modeling technology are the following:

Advantages:

- Short learning curve

- What-You-See-Is-What-You-Get

- Many additional ways to create and edit geometry

- Work with geometry from any source

- Smaller file sizes

Cons (Challenges)

- Many immature representations of direct modeling on the market

- Less optimized for design optimization and automation

- No parent/child relationship, i.e. no inherent feature to feature associativity.

The most relevant characteristics of parametric and direct modeling methods are summarized in Table 9. 
Table 9. Parametric Modeling vs. Direct Modeling

\begin{tabular}{|lcc|}
\hline Easy to learn and use & Parametric & Direct \\
\hline WYSIWYG & No & Yes \\
\hline System response time when changes occur & No & Yes \\
\hline Possibility to specify design intent & Yes (features) & Fast \\
\hline Automatic recognition of design intent & Simple features only & No \\
\hline Direct editing & Limited & Full \\
\hline Parametric editing & By history tree & Step by step \\
\hline Editing imported geometry & No & Yes \\
\hline
\end{tabular}

Some hybrid approaches such as variational direct modeling [Ushakov, 2008] have been suggested. By combining direct modeling operations with history-based technology, modifications can be made directly to the geometry without having to rollback or edit the feature history. There's no recalculation or regeneration of the model's geometry. Although hybrid modeling approaches are still in their infancy, many parametric CAD vendors have been gradually adding more and more direct modeling or "freeform" features to their systems [Tornicasa \& Di Monaco, 2010].

In general, direct modeling is more suitable for situations where front loading a design with robust design intent does not yield long-term value and where speed is more critical than highly parameterized and structured models (such as during conceptual design stages). Other situations where direct modeling may be appropriate include scenarios where unpredictable late-stage changes happen often and where product lifecycles are short, i.e.; little payoff for the investment in the structured/ordered model.

Parametric modeling is better suited to those jobs where the designer is given strict engineering criteria to meet exacting design aesthetics, performance metrics, and manufacturing criteria. The added effort and upfront planning is justified to deliver these downstream benefits. It is in the context of parametric modeling, particularly in terms of communication of design intent, that this dissertation is focused and to which the remaining of this chapter is devoted. 


\section{Feature-Based Parametric Solid Modeling}

Feature-based is a term used to describe the various components of a 3D model. For example, a part can consist of various types of features such as holes, ribs, fillets, and chamfers. Parametric refers to the ability to define constraints and relationships among geometric entities that can change the model's geometry when their values are modified (dimensional adjustability). Constraints can be dimensional, geometric, or algebraic (equations). This technique allows designers to go back to an earlier stage in the design and make changes to the model by editing a sketch or changing some dimensions. In this regard, a parametric solid model can be considered an intelligent representation of a part. Therefore, it is important to analyze and plan every modeling step beforehand to determine the most efficient sequence of features. Poor modeling strategies will result in parts that take longer to create and are difficult to modify. Features should be created to allow for maximum part flexibility and variation [Hartman, 2005].

The steps to create parametric solid models are similar across CAD packages. Models start with a 2D sketch that is typically not drawn to actual size. It is literally a rough approximation to the final shape. Next, a set of constraints and dimensions are applied to the sketch. As these constraints are applied, the sketch will change size and shape, automatically adjusting to the new values. This finished constrained sketch, commonly known as profile, will be the basis to create 3D geometry. Finally, the profile is turned into a $3 \mathrm{D}$ feature by a 3D operation such as extrude or revolve. New features are now added to the model by connecting them to existing features and repeating the same process (2D sketch, constraints, 3D operation).

In a history-based parametric system, three-dimensional features are added to the model in an associative relationship (parent/child) with the feature(s) they are immediately connected to [Hanratty, 1995], which results in a treelike structure where every node represents a feature and the design intent of the model is implicitly represented by these relations. This tree structure is known as design tree, feature tree, or history tree (see Figure 11). 


\section{$\sqrt{2}$ Boss-Extrude1 \\ $\sqrt{2}$ Boss-Extrude2 \\ Mirror1 \\ 局 Cut-Sweep1 \\ 国 Cut-Extrude1 \\ 局 Boss-Extrude3 \\ 圆 Cut-Extrude2 \\ 司 Cut-Extrude3 \\ (1) Fillet1}

Figure 11. Sample design tree in DS SolidWorks ${ }^{\circledR}$

\subsection{Modeling Strategy}

To fully benefit from history-based parametric CAD modeling, the strategy used to create $3 \mathrm{D}$ models must express the manner in which the designer expects the model to behave under certain circumstances (i.e. engineering changes), and the effects of this behavior on all aspects of the model and other components with which it interacts (assemblies) [Anderl \& Mendgen, 1998; Bertoline \& Wiebe, 2002]; Hanratty, 1995]. However, there are so many possibilities for designing a product with parametric CAD systems that not all provide the same flexibility and robustness on CAD models and make it possible to obtain the benefits promised by the parametric approach.

Since only good modeling methods can guarantee truly adaptive products, many corporations often describe their own modeling strategies in the form of CAD guidelines. The most efficient guidelines also integrate knowledge of the product development process.

In general, the selection of a modeling strategy depends on factors such as:

- Design requirements: functional requirements and how they should be defined using parent-child relationships.

- Potential areas of change: design aspects that are likely to change, and extent and impacts of such potential changes.

- Information availability: analysis of all available data about the component that will be designed.

- Manufacturing requirements: definition of dimensional and geometric tolerances. 
The modeling strategy will determine:

- Type of commands and features to be used (i.e. primitive functions, Boolean operations, sketches, etc.).

- Constraints (dimensional, geometric, algebraic) that control the model geometry.

- Relationships and sequence of the CAD functions.

- Relationships with other components (i.e. links to other parts).

The selection of a good modeling strategy becomes critical in the design of complex parts. In this regard, the complexity of a CAD modeling task is related to the complexity not so much of the product but of the development process itself [Bodein et al., 2014].

\subsection{Modeling Example}

As an example, the modeling process of a mechanical part consisting of a flange with a certain thickness and a series of six through holes along the perimeter is illustrated. The modeling strategy is described in Table 10 (dimensions and constraints have been intentionally omitted for clarity).

The true power of parametric modeling shines through when design changes need to be made. For example, in the flange used in Table 10, the circular pattern depends on the cut. In other words, the circular pattern is a child feature of the cut, the parent feature. Because modifications propagate downstream, this dependency allows designers, for example, to modify the diameter of all holes in the entire pattern (child) by simply editing a dimension in the cut (parent). This process is shown in Table 11. 
Table 10. Modeling steps for sample part

\begin{tabular}{|c|c|c|}
\hline Modeling step & Part status & Design tree \\
\hline $\begin{array}{l}\text { 1. Create initial } 2 D \text { profile, an } \\
\text { L-shaped sketch, and a } \\
\text { construction line }\end{array}$ & & $\begin{array}{l}\text { Flange } \\
\mathrm{L}_{\text {Sketch1 }}\end{array}$ \\
\hline $\begin{array}{l}\text { 2. Create initial feature by } \\
\text { revolving profile } 360 \text { degrees }\end{array}$ & & $\begin{array}{l}\text { Flange } \\
\text { L REVOLVE } \\
\text { L Sketch1 }\end{array}$ \\
\hline $\begin{array}{l}\text { 3. Create new sketch (circle) on } \\
\text { surface }\end{array}$ & & $\begin{array}{l}\text { Flange } \\
\text { L REVOLVE } \\
\begin{array}{|l}\text { L Sketch1 } \\
\text { Sketch } 2\end{array}\end{array}$ \\
\hline $\begin{array}{l}\text { 4. Cut hole from previous } \\
\text { sketch }\end{array}$ & & $\begin{array}{l}\text { Flange } \\
\text { L REVOLVE } \\
\begin{array}{c}\text { L Sketch1 } \\
\text { CUT } \\
\text { L Sketch } 2\end{array}\end{array}$ \\
\hline $\begin{array}{l}\text { 5. Apply circular pattern (six } \\
\text { equally-spaced instances) to } \\
\text { previous cut feature }\end{array}$ & & 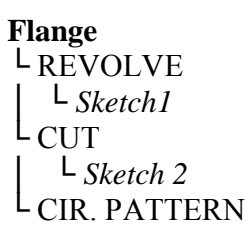 \\
\hline 6. Apply fillet to bottom edge & & $\begin{array}{l}\text { Flange } \\
\text { L REVOLVE } \\
\begin{array}{|l}\text { L Sketch } 1 \\
\text { CUT }\end{array} \\
\text { L Sketch } 2 \\
\text { CIR. PATTERN } \\
\text { L FILLET }\end{array}$ \\
\hline
\end{tabular}


Annotation Mechanisms to Manage Design Knowledge in Complex Parametric Models and their Effects on Alteration and Reusability

Table 11. Hole alteration steps for sample part

\begin{tabular}{|c|c|c|}
\hline Modeling step & Part status & Design tree \\
\hline $\begin{array}{l}\text { 1. Edit Sketch } 2 \text { to modify } \\
\text { circle diameter. }\end{array}$ & & $\begin{array}{l}\text { Flange } \\
\text { L REVOLVE } \\
\begin{array}{|l}\text { L Sketch } 1 \\
\text { CUT } \\
\text { L Sketch } 2 \\
\text { CIR. PATTERN } \\
\text { L FILLET }\end{array}\end{array}$ \\
\hline 2. Change diameter dimension & & $\begin{array}{l}\text { Flange } \\
\text { L REVOLVE } \\
\begin{array}{|l}\mathrm{L} \text { Sketch } 1 \\
\text { CUT } \\
\quad \text { S Sketch 2 } \\
\text { CIR. PATTERN } \\
\text { L FILLET }\end{array}\end{array}$ \\
\hline 3. Rebuild Model & & 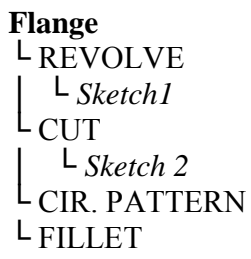 \\
\hline
\end{tabular}

\section{Internal Representation of Parametric CAD models}

The design tree is the most important editing element available in parametric modeling packages. The design tree is not a tool itself, but a representation of all the steps and operations performed to create a specific model. As new features are created, they are sequentially inserted at the bottom of the design tree. The main advantage of the design tree is that the user can go back to any specific point in the design and edit a particular feature or sketch. If dependencies and constraints remain consistent, changes will propagate to child features and the entire model will update automatically.

Despite the convenience of this mechanism, from a user perspective it is difficult to visualize feature interdependencies in the design tree. Although most CAD packages allow designers to select a specific feature node from the design tree and query its dependencies (the result is typically two lists of nodes with parent and child features), an overall view of the model's dependencies is often not available. 
Feature interdependencies can be understood as graph structures or design structure matrices (DSM), as illustrated in Figure 12. In the first case, the CAD model is represented as a directed graph, where every feature of the model is stored as a node, and every parent-child relation is represented with a directed edge from the parent to the child node.

A Design Structure Matrix, or Dependency Structure Matrix, (DSM), is a method originally introduced by [Steward, 1981] for representing and analyzing system models in a variety of application areas. It is a square matrix (i.e., it has an equal number of rows and columns) that shows relationships between elements in a system. In the context of parametric models, a binary matrix can be used because it can represent the presence or absence of a relationship between pairs of features in a model. This matrix is described as follows:

- Features of the model are placed down the left side of the matrix as row headings and across the top as column headings in the same order.

- If there exists an edge (parent-child relation) from node $i$ to node $j$, then the value of element $i, j$ (row $i$, column $j$ ) is 1 . Otherwise, the value of the element is zero.

The diagonal elements of the matrix do not have any interpretation in describing the system, so they are usually either left empty or blacked out, although many find it intuitive to think of these diagonal cells as representative of the nodes themselves. For example, the graph structure of the flange model from Table 9 as well as it DSM representation are shown in Figure 12.

Using a dependency graph or a DSM as input, a number of indicators and complexity metrics such as dependency ratio, average number of dependencies per node (or identifying the node(s) with highest number of dependencies) can be obtained. More sophisticated techniques such as partitioning, tearing, banding, and clustering can also be applied to the DSM to identify problematic nodes or optimize the model structure. 


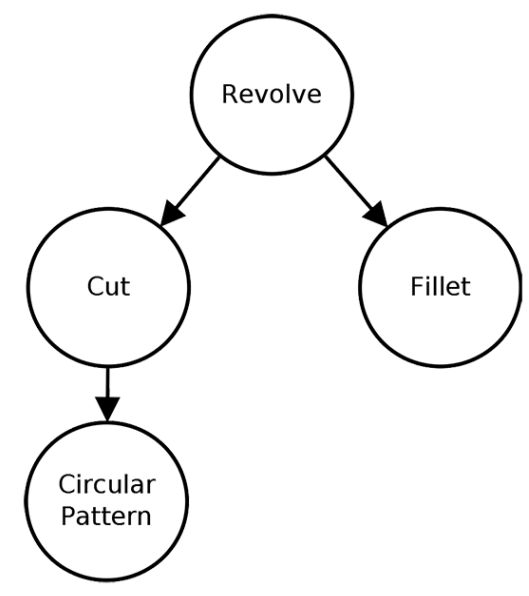

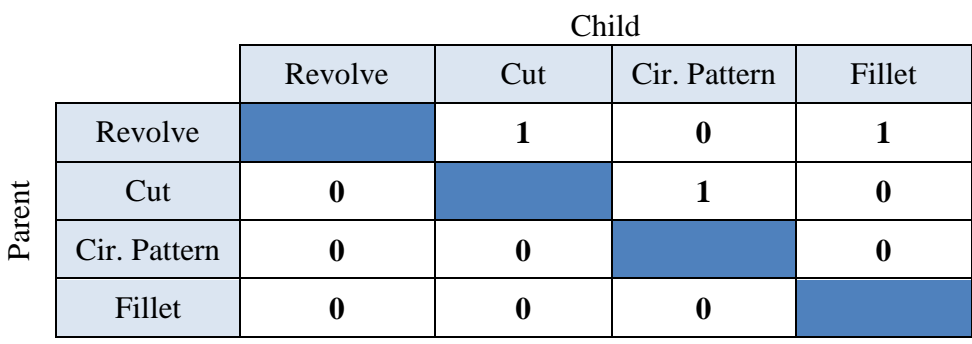

Figure 12. Graph representation (top) and DSM (bottom) of the flange model

\subsection{Complexity Metrics}

Once the DSM containing the feature interdependencies found in the model is generated, the complexity of the matrix can be calculated. A number of graph complexity metrics have been suggested [Bashir \& Thomson, 2001; Mathieson \& Summers, 2010; Shah \& Runger, 2011], some of them only useful in certain domains.

Size, or total count, is the most intuitive metric used in complexity measurement. It is based on the count of some classification of the object (number of features or number of dependencies, in the case of a parametric CAD model). In general, as size increases so does complexity [Shah \& Runger, 2011]. It must be used carefully, as size is a nonlinear complexity metric [Barclay \& Dann, 2000] (i.e. when the count is low, the addition of one more is significant, while the opposite is true of high-count systems). 
Decomposability metrics such as the algorithm by [Summers \& Ameri, 2008] measures the difficulty of a disassembling a system. Each additional step required to decompose a system is considered to increase complexity.

Centrality, the relative importance of nodes within a graph, includes a set of complexity metrics commonly used in network analysis [Koschutzki et al., 2005]. Betweenness centrality, for example, measures the number of shortest paths on which a node occurs [Freeman, 1977] and the clustering coefficient measures the degree to which nodes are grouped within the graph (percentage of nodes to which a given node is connected and which are connected to each other).

\subsection{Software Prototype}

In order to perform a reasonable comparative study of modeling methodologies, a software tool with a set of metrics was developed as part of this doctoral research. The system was implemented as a module that can directly analyze the solid models created by the commercial CAD software Dassault Systemes SolidWorks ${ }^{\circledR}$. This package was selected for availability reasons and the familiarity with the SolidWorks built-in Application Programming Interface (API) and development tools.

The tool is structured in three parts. First, calculation and visualization of the DSM is performed based on the parametric features of the CAD model. Using a bi-dimensional matrix data structure to store de DSM, the model's design tree is traversed by querying every feature to obtain its parent nodes. If the feature has no parent nodes, the value of the corresponding cell is set to zero; otherwise cells corresponding to the each parent node are set to "1." Since the base feature of the model (the one that is created first) is typically built based on one of the three primary orthographic planes, three nodes representing these three planes have been included in the DSM.

A second component provides a descriptive view of features' interconnectedness by calculating the number of direct child nodes of every feature. This value provides a numerical indicator to determine the most dependable nodes in the tree.

Finally, a set of simple metrics are presented to provide an overview of the complexity of the design tree. Metrics include total features, total dependencies, nodes with no child dependencies, and average number of child dependencies per parent. The plug-in was developed so that new metrics can be easily incorporated. Additionally, the DSM can be exported as a Comma Separated Value (.csv) file for its use in other applications such 
Annotation Mechanisms to Manage Design Knowledge in Complex Parametric Models and their Effects on Alteration and Reusability

as Microsoft Excel. The module's interface as well as the DSM and results for the flange model from Table 9 are shown in Figure 13.

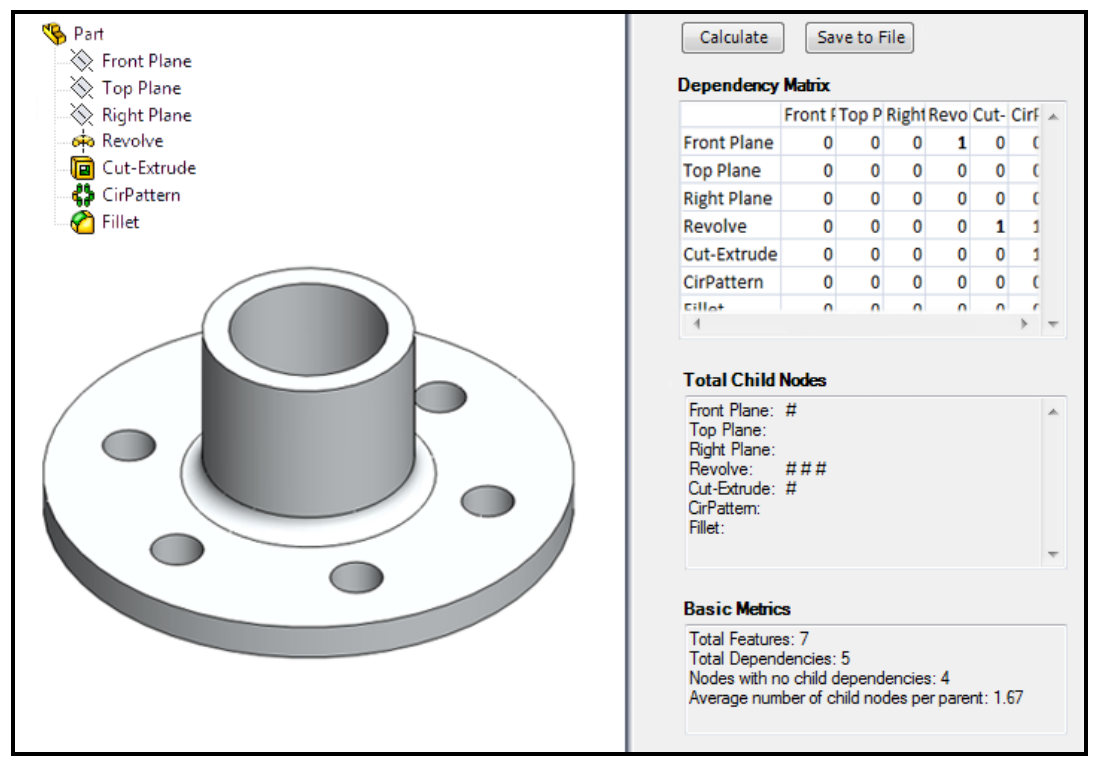

Figure 13. SolidWorks $®$ module to visualize and process DSM

The value of the tool can be appreciated when a more sophisticated model is tested. In the example shown in Figure 14, a V8 intake manifold obtained from the free CAD library GrabCAD (www.grabcad.com), we can clearly see that there are two critical features, Boss-Extrude3 and Boss-Extrude7, each with significantly more child nodes that the remaining features.

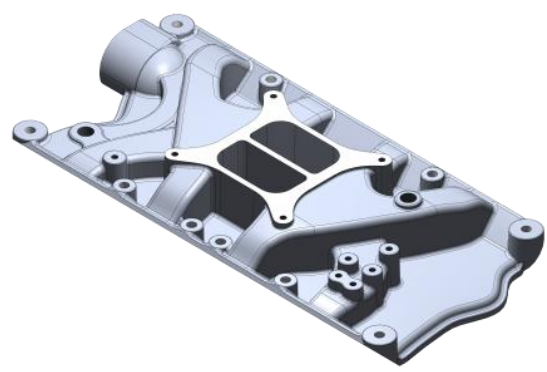

Boss-Extrude3: 55 child nodes Boss-Extrude7: 37 child nodes

total feat. $=108$

total dep. $=270$

nodes with no child dep. $=57$

average number of child dep. per parent $=5.3$

Figure 14. Complex model (left) and related information from module (right) 


\section{Case Study: A Comparison of Modeling Methodologies}

The three methodologies discussed in the previous sections provide formal mechanisms to model complex parts in a structured manner. However, the strategies to accomplish this task vary significantly, which suggests that some methodologies may be more appropriate than others for creating certain models.

Although all three methodologies have their groups of followers that claim the benefits and advantages of their preferred method over the others, there is a lack of comparative studies in the technical literature that provide objective and conclusive data that would allow us to decide which methodology is more efficient and under what circumstances.

The long-term goal of our study is to examine the factors that can help designers determine the most suitable methodology for creating a specific CAD model. As a first step toward that goal, we selected a simple part (see Figure 15) and created three CAD models using each methodology. Their corresponding design trees are illustrated in Figure 16.
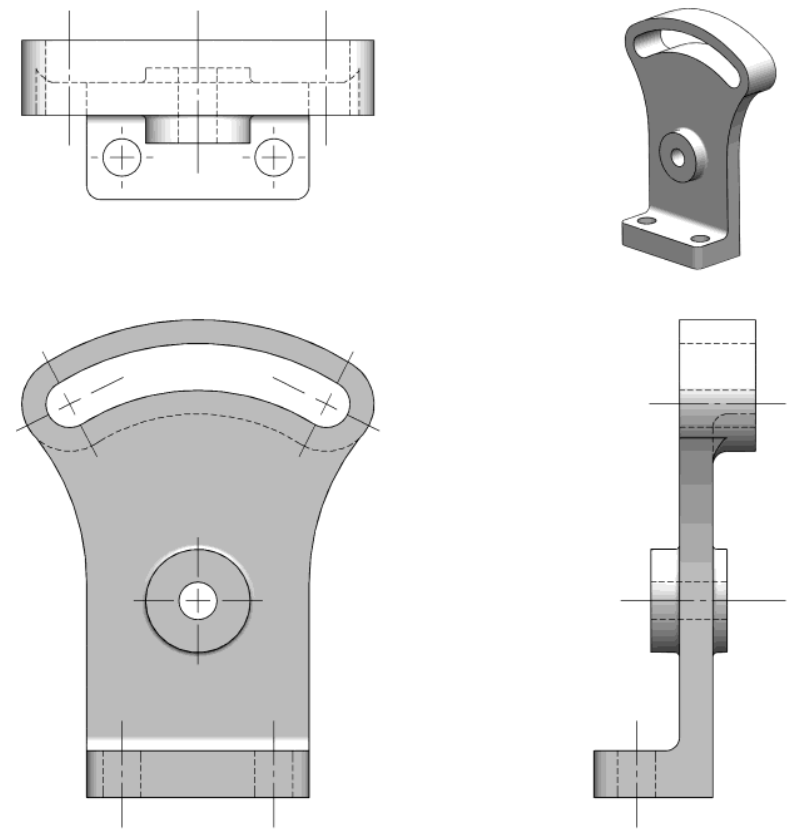

Figure 15. Part used for comparison of modeling methodologies 
Annotation Mechanisms to Manage Design Knowledge in Complex Parametric Models and their Effects on Alteration and Reusability

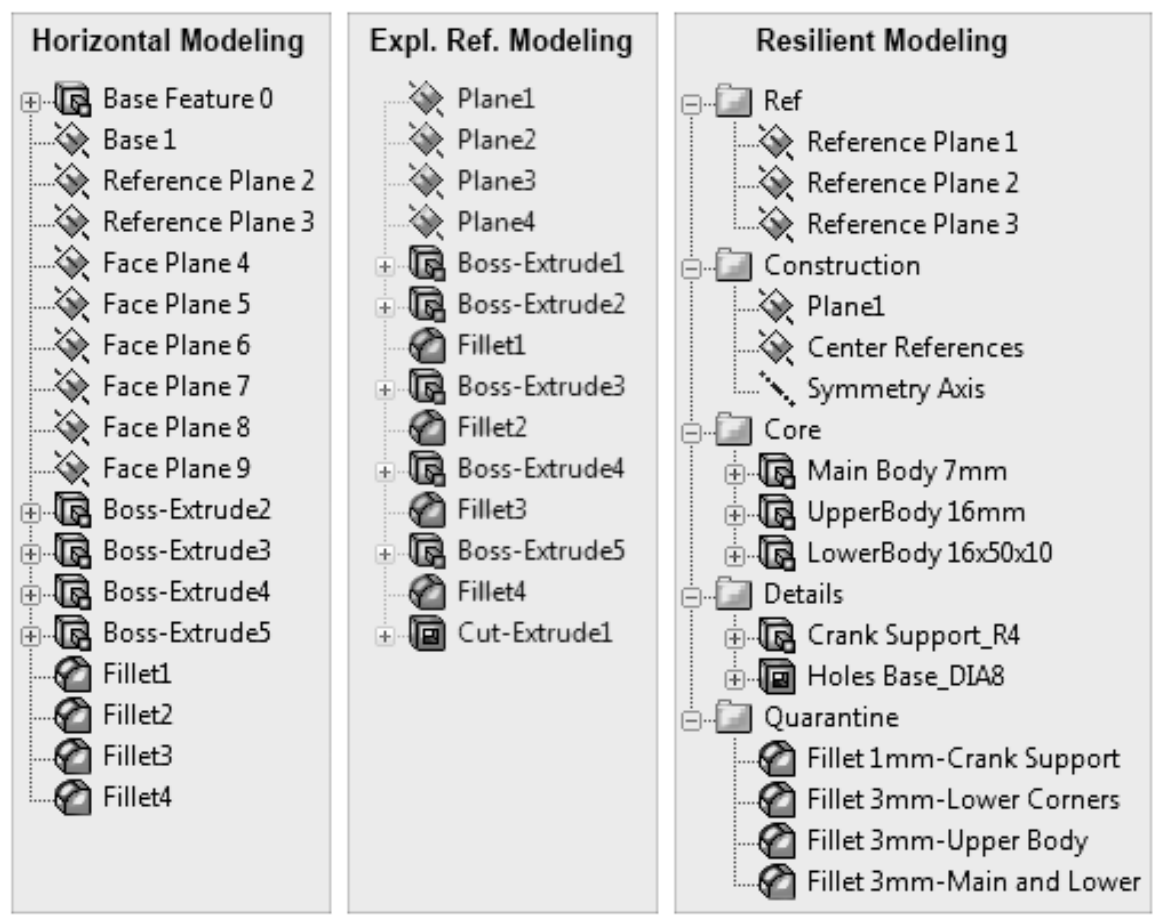

Figure 16. Design trees according to the different methodologies

To compare the complexity levels of the model for each methodology, it is necessary to analyze the interdependencies between the feature nodes that are implicit in the design tree. The DSMs of the model used in our study are shown in Figure 17 and the dependency graphs, in Figure 18. Dependencies that involve two dimensional sketches have been omitted for clarity.

In terms of modeling, the horizontal methodology requires the creation of numerous datum planes, even before generating any solid bodies, although the number of features necessary to define actual geometry remains relatively low when compared to the other two methodologies. The resilient modeling strategy requires the creation of additional items in the tree (containers or groups) but the structure is well organized and easy to follow, particularly when the naming guidelines are also applied. 


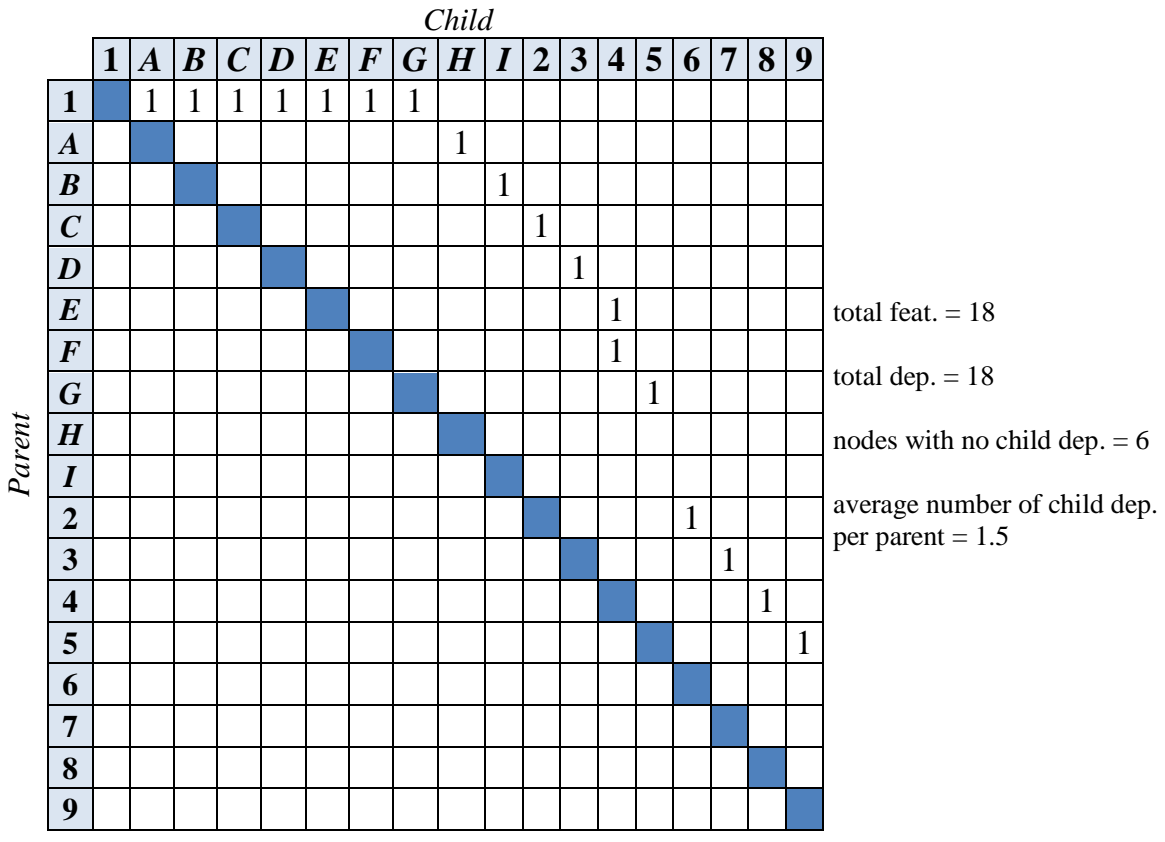

HORIZONTAL

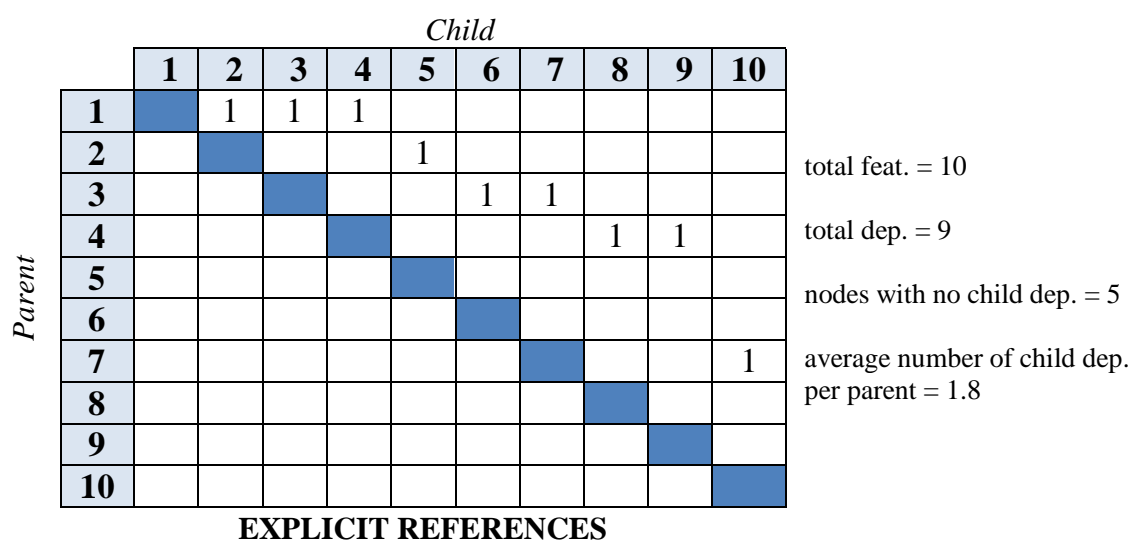




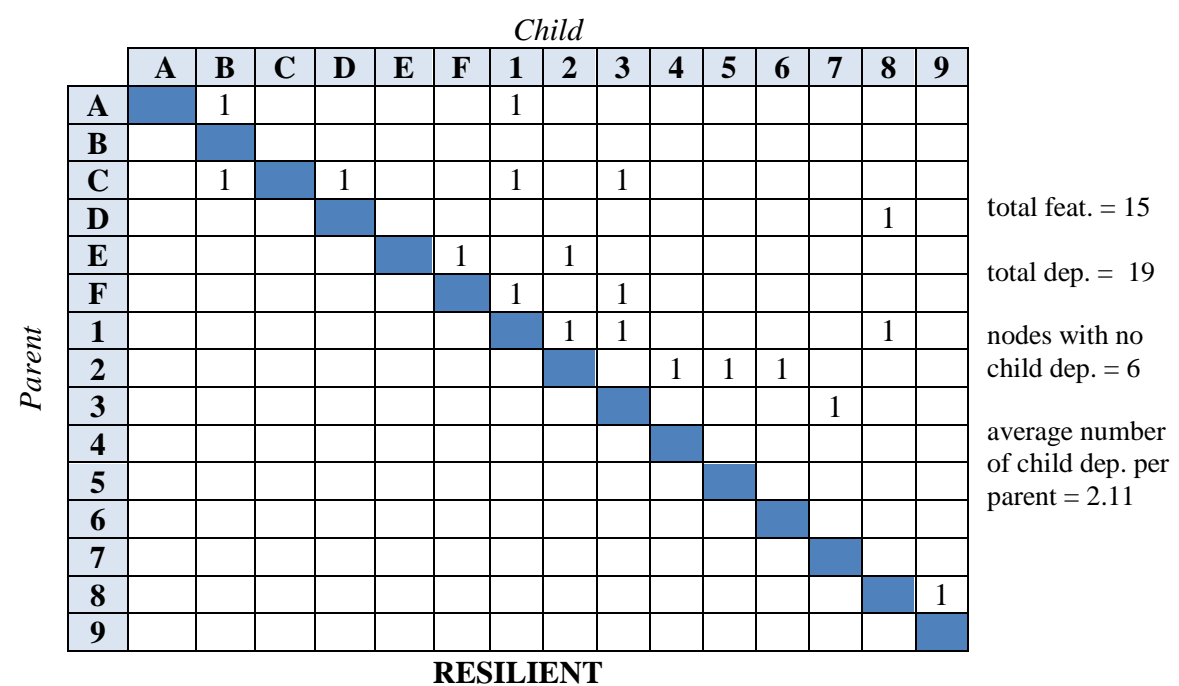

Figure 17. DSMs of the design trees for the methodologies used in the comparative study

When the horizontal modeling methodology is used, the geometry of the model becomes easy to alter, since all features behave as independent elements within the structure. Likewise, the two-dimensional sketches can also be edited without major effects on other aspects of the model. Virtually all solid bodies can be altered independently by accessing the reference geometry used to define their position. However, the horizontal methodology is not the most intuitive modeling strategy. Particularly, the intricate hierarchies used to define the reference geometry structures and the planes are not easy to understand, especially for users that are not familiar with this method. Therefore, a certain level of proficiency and effort is required to create the auxiliary structures that will support the features of the model. In addition, this methodology may not be appropriate in certain design scenarios, specifically those that involve highly complex and adaptable models, as an automatic or semi-automatic propagation of changes may be a desirable requirement. 


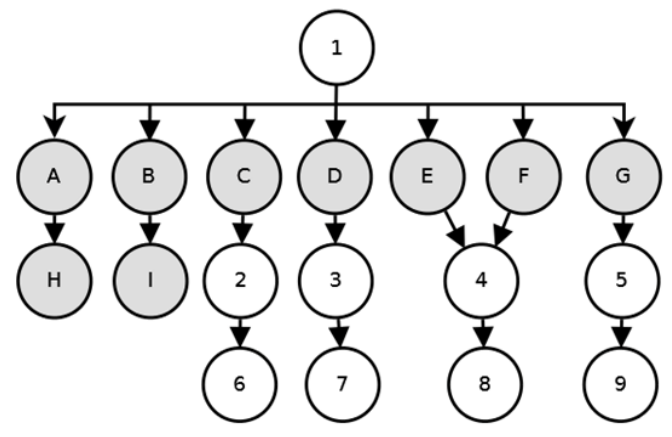

Horizontal

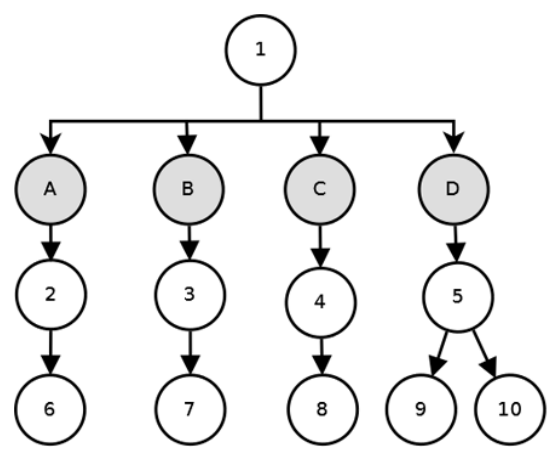

Explicit References

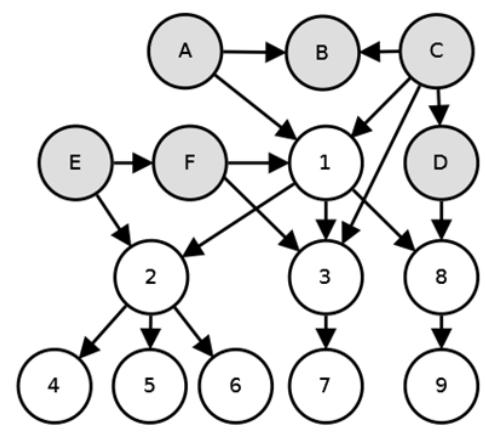

Resilient

Figure 18. Graph representation of the design trees for the methodologies used in the comparative study (nodes in grey represent reference geometry). 
When the explicit references methodology is used, designers face different challenges. In the model used in this study, the graph structure is clearly much simpler than in the previous case, which may be an advantage for designers that did not participate in the original modeling of the part or are not familiar with the process. However, this structure may be problematic when a feature located high in the tree (closer to the root node) is edited or removed, as it can cause errors that propagate down to the child nodes resulting in an unstable model. In the example shown in Figure 18, removing node 3 has a direct impact on three features (one of which is also a parent node). Part of this problem can be mitigated by adding reference geometry to serve as parents of specific feature nodes.

The resilient modeling strategy is an intuitive and well organized approach both in terms of model understanding and flexibility. The checklist provided by the RMS offers thorough standardized modeling guidelines and naming conventions that allow designers to quickly identify and understand the modeling process. This structure also facilitates information exchange among different members of a CAD team. Although the resulting feature graph may initially look cluttered and disorganized (see Figure 18), it becomes more manageable when reference geometry nodes (shown in grey) are omitted. In this case, the model is represented by a simpler structure with a high level of flexibility that can react and adapt to a large number of design changes with relatively little effort, even when those changes involve parent nodes. In the example shown in Figure 18, removing node 3 only affects one node, which is a simple fillet feature with no child nodes that can be easily fixed.

\section{Concluding Remarks}

In this chapter, a review of parametric CAD modeling was presented. The internal structure of a parametric model was studied in terms of feature interdependencies, and a software prototype was developed as an add-in for a commercial CAD package (DS SolidWorks ${ }^{\circledR}$ ) to visualize a model's DSM and evaluate model complexity. This tool was used to compare three publicly available parametric modeling methodologies: Delphi's horizontal modeling, explicit reference modeling, and resilient modeling. The strategies were evaluated side by side using a sample CAD model and analyzing the internal structure of the design trees.

In general, all methodologies offer advantages and disadvantages, so it is difficult to provide any objective recommendations as to which methodology 
is more efficient, particularly if we try to base our decisions on the one CAD model used in this study. In fact, some weaknesses in one methodology often become strengths in others. Nevertheless, some determining factors were identified:

- The horizontal modeling strategy provides the most flexible type of models at the expense of eliminating the functionalities and characteristics that make a CAD model truly parametric. In addition, the intricate structure of reference elements may dissuade designers from adopting this strategy in their regular practices.

- The explicit references modeling methodology produces a simple and integrated model structure, but it can be difficult to alter in certain situations, particularly if the node that needs to be changed has many dependencies. Although some of the negative aspects can be mitigated by using reference geometry, it can also increase the complexity of the graph.

- The resilient modeling strategy is effective, easy to follow, and well organized, despite few reference nodes required to minimize dependencies. It also requires users to be familiar with the standard features groups and checklist. Further studies with more complex models are required to draw more conclusive results.

In order to identify specific geometric characteristics in designs that can aid users in selecting the most efficient strategy for creating CAD models, a more extensive and comprehensive analysis of these methodologies is needed. This analysis requires the use of CAD models with different levels of complexity and a greater number of features. Methodologies would be evaluated by examining the effects of altering parent nodes and determining how easy it is to recover from rebuilding errors. This data can provide valuable information to make informed decisions about modeling methodologies and best practices. Nevertheless, this comprehensive review is out of the scope of this dissertation.

Unfortunately, a good modeling methodology alone does not guarantee CAD efficiency and reusability. Once a part reaches a certain level of complexity in terms of number of features and interdependencies, guidelines and modeling strategies are insufficient. This situation can easily be observed in practical scenarios. As stated by [Bodein et al., 2014], the same part modeled by two different expert designers will likely have a different construction history (sequence of features) and ability to adapt to design changes. 

their Effects on Alteration and Reusability

Therefore, it is necessary to implement other mechanisms to aid designers in creating more reusable models. In the following chapters, the concept of product design communication is examined and the architecture of an annotation-center design intent strategy is presented as a complementary resource to increase reusability of large complex models and enhance collaboration. 


\section{Chapter IV \\ Communication using Design Annotations}

\section{Introduction}

In this chapter, we lay the foundation for understanding the potential of annotated models in the context of reusability and their limitations in terms of communication. User's performance is evaluated in simulated scenarios that involve the alteration of annotated CAD models. The objective was to find statistically significant evidence of better responses in terms of design and model quality when annotations are used to communicate design intent.

The results of a series of studies are presented. First, we hypothesize that annotations are valuable tools to provide design information when inadequate modeling assumptions can be made by designers. Second, we evaluate annotations as tools to communicate design decisions when multiple options are available.

\section{Hypothesis Definition}

The framework of this study is the application of $3 \mathrm{D}$ annotations to parametric modeling processes. The main goal is to determine whether annotated models provide a significant benefit over non-annotated models when performing tasks that require manipulation of the model's geometry. With this goal in mind, two research questions are formulated: 
$\mathrm{Q}_{1}$ : When modifying a parametric model, are annotations an effective tool to communicate specific design intent information so it is easier for designers to select the most appropriate procedure to perform such modifications?

$\mathrm{Q}_{2}$ : When critical design decisions have to be made, are annotations a valuable tool to explicitly express geometric design requirements and considerations about the model?

For $\mathrm{Q}_{1}$, two important aspects were identified: the difficulty of selecting the most appropriate solution (in terms of model flexibility and reusability) to perform a particular modification to the model when multiple options are available, and the clear understanding of the annotation information and the specific aspect of the model that it refers to. For $\mathrm{Q}_{2}$, the focus is on the quality and correctness of the model, ensuring that all design requirements expressed by the annotations are met.

Based on the previous questions and framed in terms of research hypotheses, we aim at rejecting:

$\mathrm{H}_{0(1)}$ : Annotations do not have a significant impact on communicating design intent information when altering a parametric model.

$\mathrm{H}_{0(2)}$ : When critical design decisions about a model need to be made, annotated models are not more valuable than non-annotated models in terms of communicating design requirements.

\section{Experimental Analysis}

A series of studies were conducted with a group of undergraduate engineering students with previous experience in engineering design graphics and parametric solid modeling, particularly using the CAD package SolidWorks ${ }^{\circledR}$. The experiments took place in a computer laboratory environment, where participants were equipped with a workstation and the CAD software.

Two separate experiments were devised: the first one aimed at model alteration activities, and the second focused on design tasks. For both experiments, participants were randomly divided into two groups. One group served as the control group (participants used non-annotated models to complete the tasks) and the other served as the experimental group (participants used models that were previously annotated by a member of the research team). 
In order not to give a clear advantage to the experimental group, no instructions were given regarding the existence or relevance of the annotations. None of the participants had previous experience with annotation techniques and the model was presented as an industrial component created by a professional designer, without mentioning or announcing annotations. In addition, not all annotations included in the model were relevant to the task. Some were prepared to look like personal comments and reminders. Others were more technical in nature. If the full extent of the task was known, then the designer could be informed before initiating work, and obviously perform better. We tried to minimize the amount of information by not providing annotations that give a clear and expected plan of the changes that participants would be called to make.

\subsection{Experiment 1}

The first experiment was intended to obtain new insights on CAD modeling strategies that will allow us to answer $\mathrm{Q}_{1}$ (previously discussed). The objective was to determine whether annotations are helpful in situations where inadequate assumptions are likely to be made by designers during the modeling process, but can be prevented when design information is explicitly available.

Two activities, each involving a series of alterations to an existing parametric CAD model, were designed for this experiment. The activities were presented in a classic test format, using an online testing tool. Each alteration in the sequence was stated as a separate question, requiring participants to submit their modified CAD model for each question. In order to accurately analyze the modeling process followed by the participants for the entire sequence, only one question was displayed at a time and backtracking was intentionally disabled, so participants were forced to submit a CAD model for every question before moving to the next, and were not allowed to change the answer to a question that was previously submitted.

\subsubsection{Activity 1}

A total of 104 volunteers, randomly divided into two groups of 52, participated in this activity. Participants in both groups received the same model, but a set of ten annotations was added to the model given to the experimental group. Only one of those annotations, indicating not to assume symmetrical arms, is relevant to the task. The CAD model provided to the participants is shown in Figure 19. In the case of the experimental group 
Annotation Mechanisms to Manage Design Knowledge in Complex Parametric Models and their Effects on Alteration and Reusability

(Figure 19, right), dimensions and annotations that are not relevant to the design problem have been intentionally omitted for clarity.

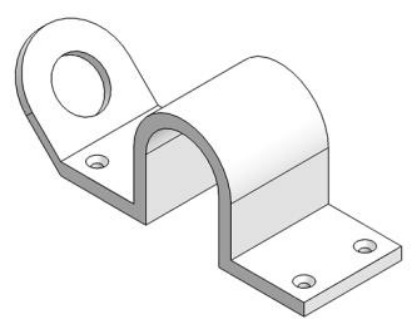

Non-annotated model (Control Group)

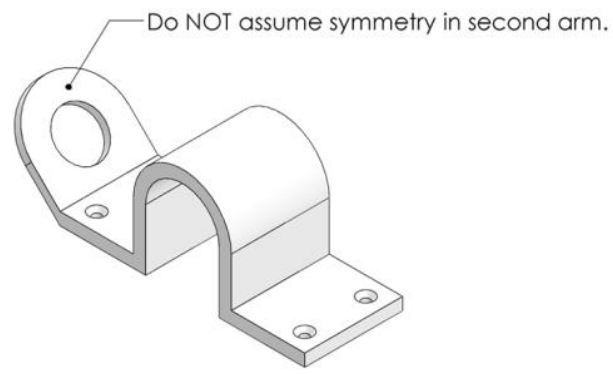

Annotated model (Experimental Group)

Figure 19. Model provided to participants for Activity 1.

The basic steps involved in creating the initial model are represented in Figure 20. Each step is the result of applying a modeling operation using the CAD package.

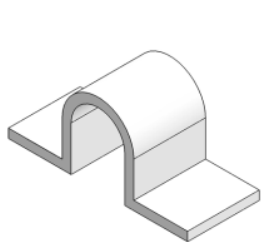

1. Base feature

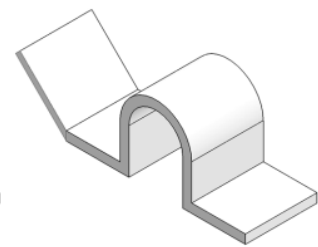

2. Extrude arm

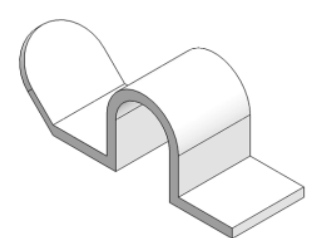

3. Round corners

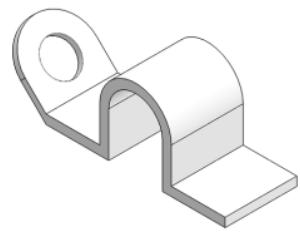

4. Cut hole on arm

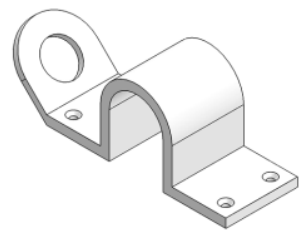

5. Cut machined holes on base

Figure 20. Creation process of original model for activity 1 
The sequence of modifications requested is illustrated in Figure 21. Tasks were presented one at a time. Therefore, participants were not allowed to see alteration $i+l$ before completing alteration $i$. The material presented to participants is available in Appendix A.
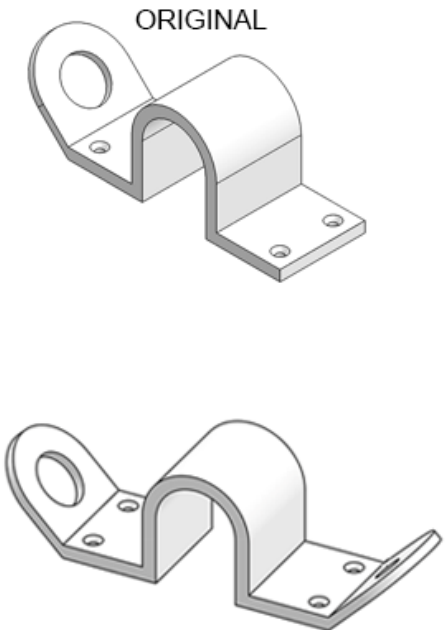

ALTERATION 2

(change hole diameter)

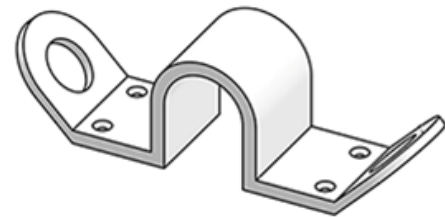

ALTERATION 1

(add second arm)

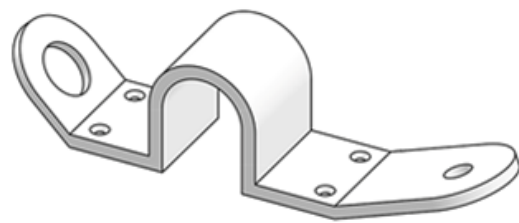

ALTERATION 3

(modify angle and size)

Figure 21 . Sequence of alterations requested for activity 1

The first modification task involves the addition of a second arm to the model, identical to the existing arm in both shape and size. There are, at least, two distinctive approaches that can be followed: using symmetry tools to mirror all features in the existing arm, or creating the second arm from scratch by defining a new sketch, extruding it, and cutting a new hole, i.e. repeating the process used to create the original arm (see Figure 22).

Intuitively, the first approach seems easier and faster than the second, as it requires fewer steps and no features need to be modeled from scratch. The result is an efficient model as long as all features in both arms remain symmetrical in new variations of the model. In other words, when selecting this approach, the designer is assuming that both arms are likely to remain symmetrical in future models, which is reasonable if "Alteration 1" is the only piece of information she possesses. Performing the first alteration to the 
Annotation Mechanisms to Manage Design Knowledge in Complex Parametric Models and their Effects on Alteration and Reusability

model by creating the second arm from scratch may not seem like an efficient strategy, as it does require a number of additional steps. However, each arm can be controlled and edited separately without affecting the other, should only one arm needed to be modified (which is precisely what happens in upcoming alterations).

APPROACH 1: MIRROR FEATURES

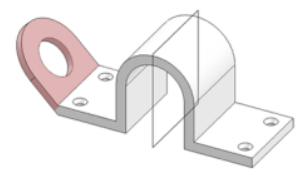

1. Select features to mirror (extrusion, fillet, and cut) and select mirror plane

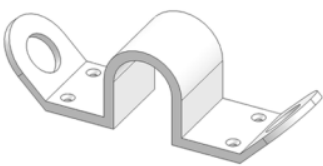

2. Mirror existing features

\section{APPROACH 2: MODEL NEW FEATURES}

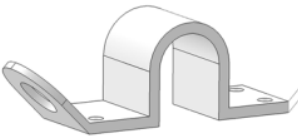

1. Create new sketch

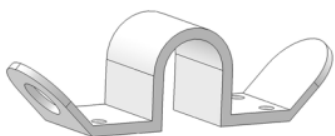

3. Fillet edges

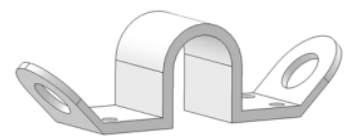

5. Cut hole

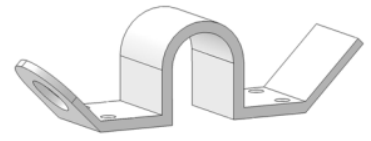

2. Extrude sketch

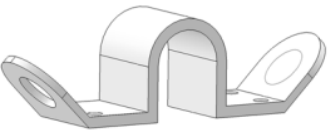

4. Create new sketch

Figure 22. Basic approaches for Alteration 1 
By analyzing the entire sequence, it seems clear that mirroring the existing arm (approach 2) might not be the best approach, since both the angle and the length of the new arm will be modified in Alteration 3. In fact, if symmetry is assumed, alteration 3 will require the suppression or deletion of all mirrored features and the creation of the new arm from scratch (which is precisely "approach 2"). If we were using a more sophisticated model with a greater number of features that depended on the new arm, performing alteration 3 after mirroring would likely cause major rebuild errors and require a significant amount of time to fix. Since none of this information is available to participants in the control group at the time they have to make a decision about Alteration 1, we hypothesized that:

- If using a non-annotated model, the majority of participants will assume symmetry and use mirror tools to perform Alteration 1 .

- If using an annotated model, the majority of participants will create the second arm by modeling the new features from scratch.

Let us define the modeling approach as the independent categorical variable $\mathrm{X}_{1}$ with values as shown in Table 12 .

Table 12. Values of independent variable $\mathrm{X}_{1}$

\begin{tabular}{|clll|}
\hline \multicolumn{1}{|c|}{$\mathbf{X}_{1}$} & Alteration 1 & Alteration 2 & Alteration 3 \\
\hline Approach 1 & Mirror arm & $\begin{array}{l}\text { Edit previous mirror } \\
\text { and create new hole }\end{array}$ & $\begin{array}{l}\text { Delete previous mirror } \\
\text { and re-model arm }\end{array}$ \\
\hline Approach 2* & $\begin{array}{l}\text { Model new } \\
\text { arm }\end{array}$ & $\begin{array}{l}\text { Edit circle in sketch and } \\
\text { update feature }\end{array}$ & $\begin{array}{l}\text { Change dimensions of } \\
\text { sketch and update feature }\end{array}$ \\
\hline Approach 3** & Mirror arm & $\begin{array}{l}\text { Delete previous mirror } \\
\text { and re-model arm }\end{array}$ & $\begin{array}{l}\text { Change dimensions of } \\
\text { sketch and update feature }\end{array}$ \\
\hline
\end{tabular}

*Most efficient approach, as previously discussed

**When alteration 2 was presented, some participants realized the need to control the two arms separately and decided to re-model the arm from scratch.

Due to the categorical nature of our data, we performed a Chi-Square Test aimed at rejecting $\mathrm{H}_{0 \text { (activity 1), defined as: }}$

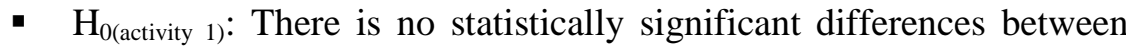
the experimental and control groups. The annotation does not contribute to the selection of an efficient modeling approach. 
Annotation Mechanisms to Manage Design Knowledge in Complex Parametric Models and their Effects on Alteration and Reusability

- $\mathrm{H}_{\mathrm{a}(\text { activity } 1)}$ : There is significant difference between the experimental and control groups. The annotation provided contributes significantly to the selection of an efficient modeling approach.

The results of our study are shown in Table 13.

Table 13. Observed values for activity 1

Approach 1 Approach 2 Approach 3 Total

\begin{tabular}{|llcll|}
\hline Control Group: Non-annotated model & 38 & 10 & 4 & 52 \\
\hline Experimental Group: Annotated model & 11 & 39 & 2 & 52 \\
\hline Total & 49 & 49 & 6 & 104 \\
\hline Chi-square=32.707, p-value $<\mathbf{0 . 0 0 1}$ & & & & \\
\hline
\end{tabular}

Based on the resulting $\mathrm{p}$-value $(\mathrm{p}<0.001)$, there is a statistically significant difference between the experimental and control groups. Even with no prior warnings, the design information provided as an annotation contributes to the selection of an efficient modeling approach.

\subsubsection{Activity 2}

A total of 77 volunteers (a subset of the group involved in Activity 1), randomly divided into two groups (control group: 38, experimental group: 39) participated in this activity. The tasks involved in this part of the experiment are similar to those proposed in Activity 1. In this case, five alterations were proposed and a number of annotations were inserted into the model provided to the experimental group. Once again, no mention of the existence or relevance of the annotations was given to the experimental group. The CAD model provided to the participants is shown in Figure 23. In the case of the experimental group (Figure 23, right), dimensions and annotations that are not relevant to the design problem have been intentionally omitted for clarity. 


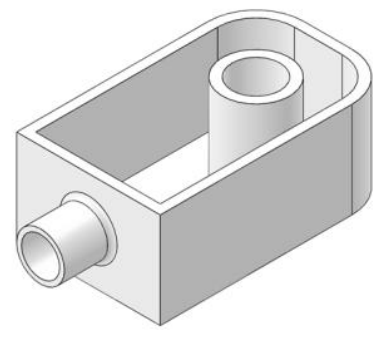

Non-annotated model

(Control Group)

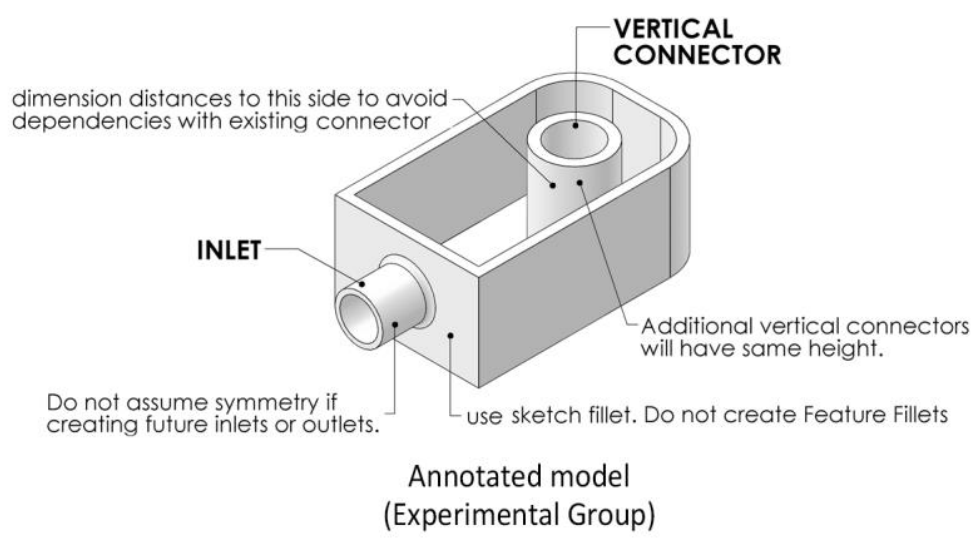

Figure 23. Model provided to participants for activity 2

The basic steps involved in creating the model for this activity are represented in Figure 24. Each step is the result of applying a modeling operation using the $\mathrm{CAD}$ package.

The sequence of modifications requested to the participants is shown in Figure 25. Similar to the previous activity, tasks were presented one at a time, i.e. participants were not allowed to see alteration $i+1$ until they completed alteration $i$. The material presented to participants is available in Appendix B. 


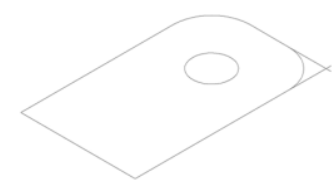

1. Initial sketch

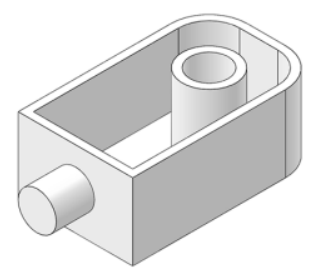

4. Extrude inlet

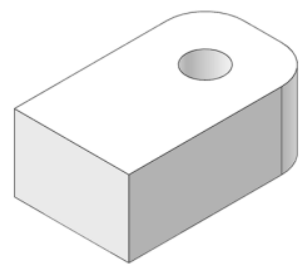

2. Base feature

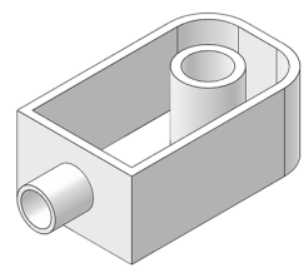

5. Cut hole

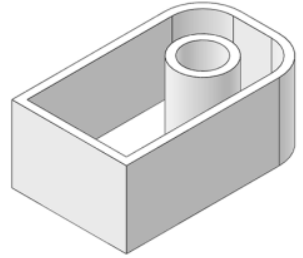

3. Shell top surface

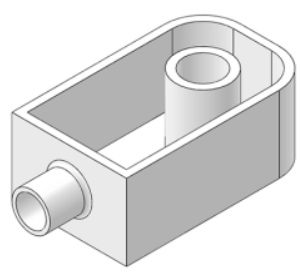

6. Round edge

Figure 24. Creation process of original model for activity 2

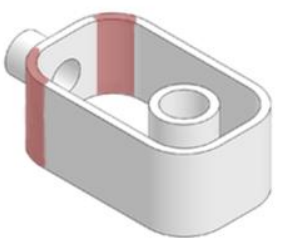

ALTERATION 1

(fillet edges)

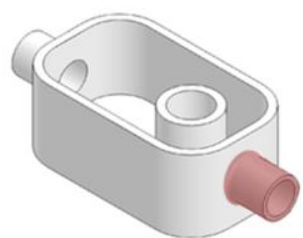

ALTERATION 2 (create outlet)

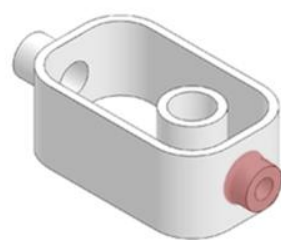

ALTERATION 3 (modify outlet)

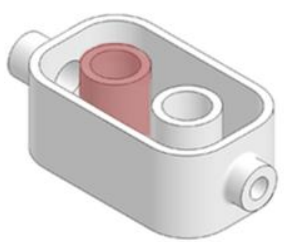

ALTERATION 4 (create new connector)

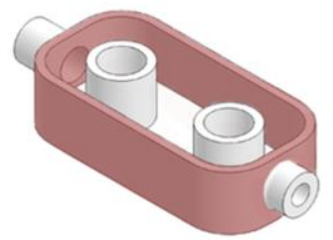

ALTERATION 5

(modify width and height)

Figure 25. Sequence of alterations requested for activity 2 
Based on the influence on later modifications and on the overall model behavior, the sequence of alterations described in Figure 25 can be divided into three tasks:

- Task A: Alteration 1 (the approach taken to perform this alteration does not affect any of the subsequent steps)

- Task B: Alterations 2 and 3 (the approach taken to perform alteration 2 affects alteration 3, but not others)

- Task C: Alterations 4 and 5 (the approach taken to perform alteration 4 affects alteration 5 , but not others)

For each task, we performed independent Chi-Square Tests aimed at

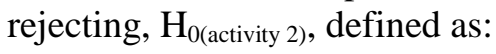

- $\mathrm{H}_{0(\text { (activity 2) }}$ : There is not a statistically significant difference between the experimental and control groups. Annotations do not contribute to the selection of a correct modeling approach.

- $\mathrm{H}_{\mathrm{a}(\text { activity 2): }}$ There is substantial difference between the experimental and control groups. Annotations contribute significantly to the selection of a correct modeling approach.

\section{ACTIVITY 2 (TASK A): FILLET CREATION}

The creation of fillets in 3D models can be performed either at sketch level or feature level. Both methods can produce models that are identical in appearance (see Figure 26). Sketch level fillets involve rounding off the corner at the intersection of two lines in a two-dimensional sketch, whereas feature level fillets create a rounded face on the part based on the selected edge. In general, feature fillets are preferred over sketch fillets, as feature fillets can be edited, deleted, or suppressed independently from the original sketch, they allow more flexibility and control over the model's corners, and they support variable radii, among other advantages. Furthermore, because other features can affect fillets, fillets are usually added toward the end of the modeling process. 


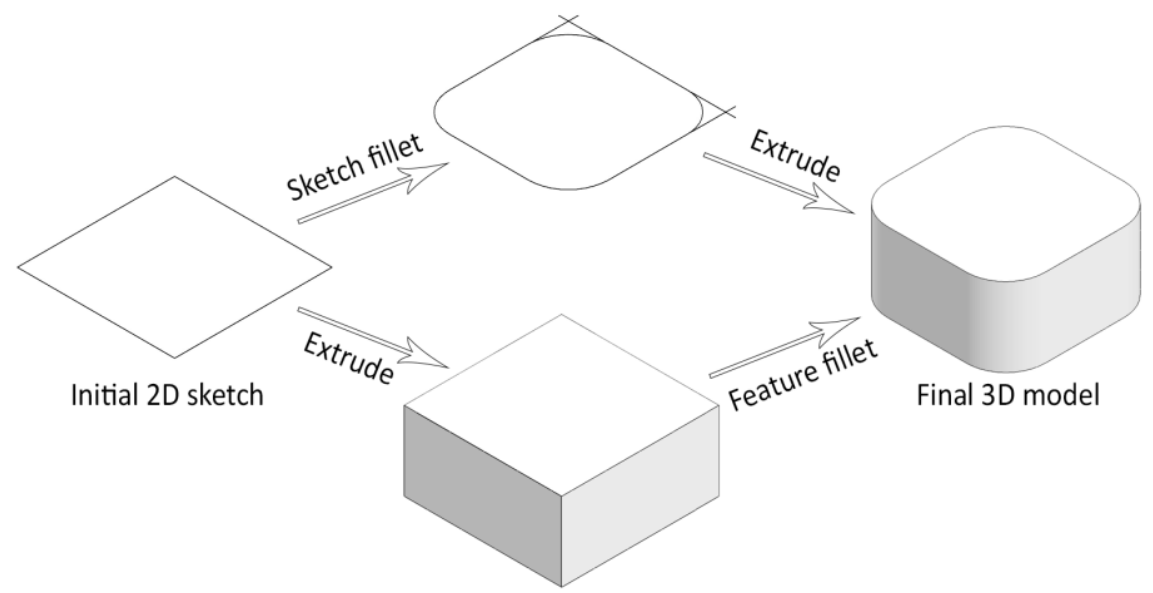

Figure 26. Two approaches to fillet creation

In our example, however, creating the fillets requested in Alteration 1at feature level produces an incorrect model. The problem is caused by the geometry of the inlet located on the external face and the shell command used to hollow out the top surface. Selecting the inner and outer vertical edges of the model to create feature fillets causes the filleted arcs to lose concentricity, creating a thicker wall on that side of the model (see Figure 27). In this situation, feature fillets are not only inefficient, but also incorrect. Therefore, sketch fillets must be used. We hypothesized that:

- If using a non-annotated model, the majority of participants will create the requested fillets at feature level, based on the preconceived advantages of this method without realizing the problematic effects in this specific situation.

- If using an annotated model, the majority of participants will create sketch fillets, preventing the problem shown in Figure 27. 

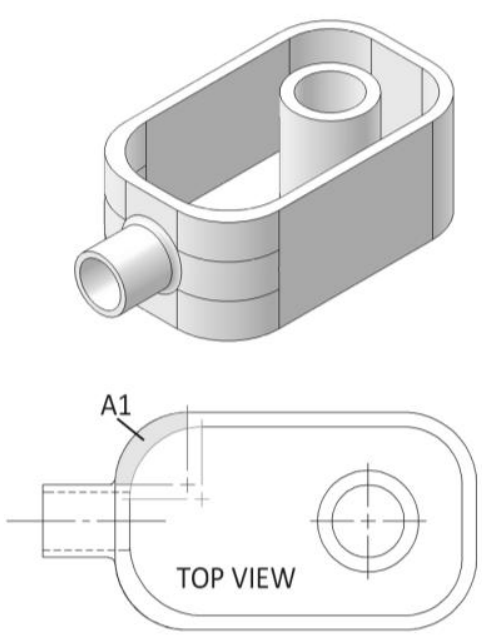

FEATURE FILLETS

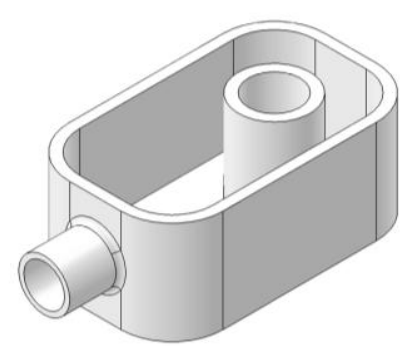

$\mathrm{A} 1>\mathrm{A} 2$

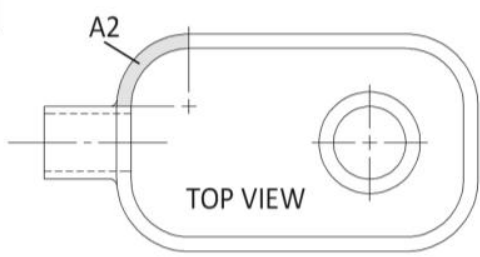

SKETCH FILLETS

Figure 27. Feature fillets (incorrect) vs. Sketch fillets

The results of our study are shown in Table 14.

Table 14. Observed values for activity 2 (fillet creation)

\section{Sketch fillet Feature fillet Total}

\begin{tabular}{|lccc|}
\hline Control Group: Non-annotated model & 5 & 33 & 38 \\
\hline Experimental Group: Annotated model & 28 & 11 & 39 \\
\hline Total & 33 & 44 & 77 \\
\hline Chi-square=27.022, p-value $<\mathbf{0 . 0 0 1}$ & & & \\
\hline
\end{tabular}

Based on the resulting $\mathrm{p}$-value $(\mathrm{p}<0.001)$, there is a statistically significant difference between the experimental and control groups. Even with no prior warnings, the design information provided as an annotation contributes to the selection of an efficient modeling approach. 
Annotation Mechanisms to Manage Design Knowledge in Complex Parametric Models and their Effects on Alteration and Reusability

\section{ACTIVITY 2 (TASK B): OUTLET}

The tasks in this group are similar to the ones designed for activity 1 . First, the addition of an outlet, identical to the existing inlet in both shape and size, is requested. There are two approaches that can be followed: using symmetry tools to mirror all features in the inlet, or creating the outlet from scratch by defining a new sketch, extruding it, and cutting a new hole, i.e. repeating the process used to create the original inlet.

Once again, the first approach seems a better option, as long as all features in the outlet remain symmetrical to the inlet in new variations of the model. As we observed in activity 1 , this is a reasonable assumption when the designer only possesses a limited piece of information. Modeling the outlet from scratch, however, allows us to control the outlet independently from the inlet, which is helpful when future modifications such as "Alteration 3" need to be performed. The value of modeling the outlet from scratch instead of mirroring the existing inlet becomes more obvious when more drastic modifications are necessary, such as changing the location of the outlet, creating multiple outlets on the same surface, or significantly redesigning its shape. For this task, we hypothesized that:

- If using a non-annotated model, the majority of participants will assume symmetry and use mirror tools to create the outlet features.

- If using a model that was previously annotated with relevant design information, the majority of participants will model the outlet from scratch.

We define the modeling approach as the independent variable $\mathrm{X}_{2}$ with values as shown in Table 15. The results of our study are shown in Table 16.

Table 15. Values of independent variable $\mathrm{X}_{2}$

\begin{tabular}{|lll|}
\hline \multicolumn{1}{|c|}{$\mathbf{X}_{2}$} & Alteration 1 & Description of the modeling approach \\
\hline Approach 1 & Mirror inlet to create outlet & $\begin{array}{l}\text { Delete mirror and re-model outlet with } \\
\text { new dimensions }\end{array}$ \\
\hline Approach 2* & Model outlet from scratch & Edit outlet diameter and length \\
\hline Approach 3** & Mirror inlet to create outlet & $\begin{array}{l}\text { New sketch on mirrored feature and cut } \\
\text { to reduce outlet length }\end{array}$ \\
\hline
\end{tabular}

*Most efficient approach, as previously discussed

**Approach 3 is an inefficient approach taken by some participants. In this case, updating the dimensions of the outlet requires making changes to three or more different features. 
Table 16. Observed values for activity 2 (outlet creation)

Approach 1 Approach 2 Approach 3 Total

\begin{tabular}{|lcccc|}
\hline Control Group: Non-annotated model & 21 & 15 & 2 & 38 \\
\hline Experimental Group: Annotated model & 6 & 30 & 3 & 39 \\
\hline Total & 27 & 45 & 5 & 77 \\
\hline Chi-square=13.33, p-value $<\mathbf{0 . 0 0 1}$ & & & & \\
\hline
\end{tabular}

Based on the resulting $\mathrm{p}$-value $(\mathrm{p}<0.001)$, there is a statistically significant difference between the experimental and control groups. Even with no prior warnings, the design information provided as an annotation contributes substantially to the selection of an efficient modeling approach.

\section{ACTIVITY 2 (TASK C): VERTICAL CONNECTOR AND OVERALL $\underline{\text { SIZE }}$}

For the last two alterations, three major approaches can be taken. Two of these can be further divided based on the selection of dimensional constraints (see Figure 28). Because the overall width and height of the part will change in alteration 5, the goal is to optimize the model, so it is flexible enough to automatically adjust to the changes. In this case, since both vertical connectors will remain identical in size and symmetrical, approaches $1 \mathrm{~b}$ and 3 are the most efficient modeling strategies. Based on the information provided in the annotations for this task, we hypothesized that:

- If using a non-annotated model, the majority of participants will not select an efficient modeling strategy to create the new vertical connector.

- If using an annotated model, the majority of participants will select an efficient modeling strategy (approaches $1 \mathrm{~b}$ or 3 ) to model the new connector.

The results of our study are shown in Table 17 . 
Annotation Mechanisms to Manage Design Knowledge in Complex Parametric Models and their Effects on Alteration and Reusability

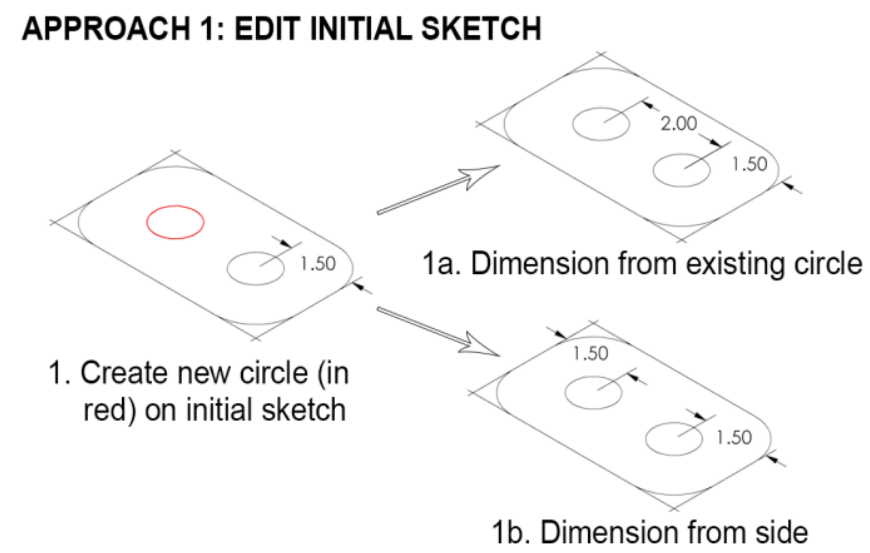

APPROACH 2: NEW SKETCH

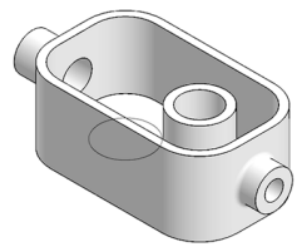

2. Create new sketch on surface

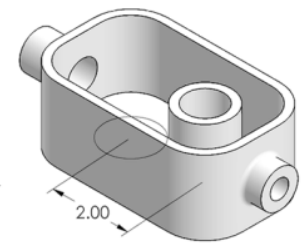

2a. Dimension from existing connector

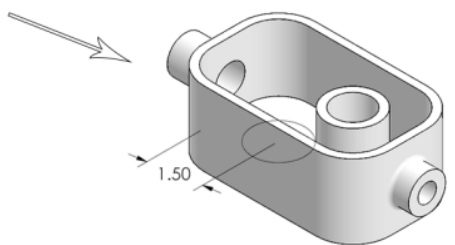

2b. Dimension from side

\section{APPROACH 3: MIRROR CONNECTOR}

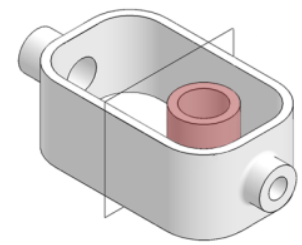

3. Mirror existing connector about plane

Figure 28. Modeling approaches to new vertical connector 
Table 17. Observed values for activity 2 (new connector)

\begin{tabular}{|lcccccc|}
\multicolumn{1}{l|}{$\begin{array}{c}\text { Approach } \\
\text { 1a }\end{array}$} & $\begin{array}{c}\text { Approach } \\
\mathbf{1 b}\end{array}$ & $\begin{array}{c}\text { Approach } \\
\mathbf{2 a}\end{array}$ & $\begin{array}{c}\text { Approach } \\
\mathbf{2 b}\end{array}$ & $\begin{array}{c}\text { Approach } \\
\mathbf{3}\end{array}$ & Total \\
\begin{tabular}{|l} 
Control Group: Non- \\
annotated model
\end{tabular} & 5 & 2 & 17 & 13 & 1 & 38 \\
\hline $\begin{array}{l}\text { Experimental Group: } \\
\text { Annotated model }\end{array}$ & 3 & 17 & 5 & 11 & 3 & 39 \\
\hline Total & 8 & 19 & 22 & 24 & 4 & 77 \\
\hline Chi-square=20.045, p-value $<\mathbf{0 . 0 0 1}$ & & & & & \\
\hline
\end{tabular}

Based on the resulting $p$-value ( $\mathrm{p}<0.001)$, there is a statistically significant difference between the experimental and control groups. Even with no prior warnings, the design information provided as an annotation contributes substantially to the selection of an efficient modeling approach.

\subsection{Experiment 2}

The goal of the second experiment is to determine whether annotations are a valuable tool to communicate requirements, constraints, and modeling considerations in situations where design decisions need to be made. Rather than making specific changes to an existing model, in this experiment participants are asked to find a solution to a design problem that can be solved by taking different approaches. A total of 104 participants (52 in the control group in 52 in the experimental group) were part of this study. The models provided to participants are shown in Figure 29.
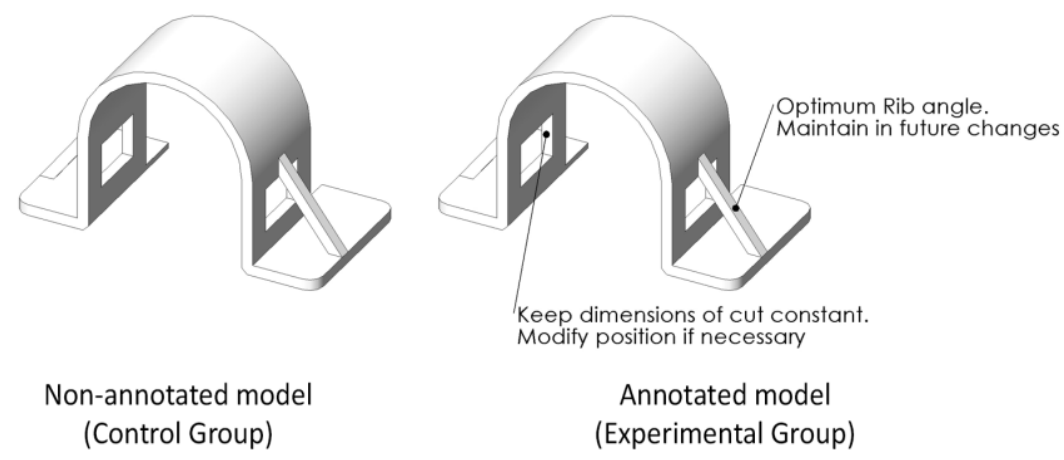

Figure 29. Model provided to participants for experiment 2 
Annotation Mechanisms to Manage Design Knowledge in Complex Parametric Models and their Effects on Alteration and Reusability

Once again, in the case of the experimental group (Figure 29, right) dimensions and annotations that are not relevant to the design problem have been intentionally omitted for clarity.

As a first step, participants were asked to change the dimensions of the side ribs from $4 \mathrm{~mm}$ to $5 \mathrm{~mm}$ (see Figure 30, left). This alteration is intentionally prepared to cause an unwanted effect in the model (see Figure 30, right). Participants were then challenged to find a solution to this undesired geometry.
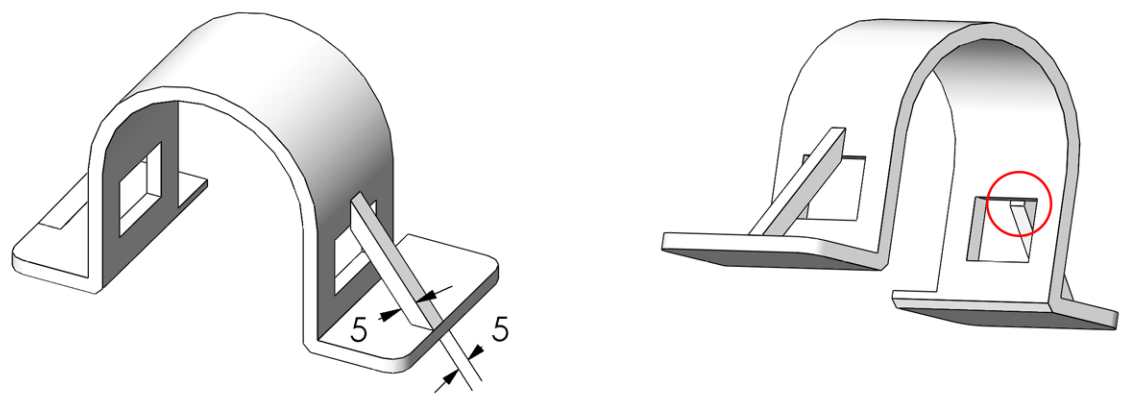

Figure 30. First alteration (left) causes unwanted effect (right, inside circle)

Three major approaches to this problem were identified: increasing the angle of the ribs, reducing the dimensions of the square cuts on both sides of the part, or changing the position of the side cuts by moving them closer to the bottom (see Figure 31).

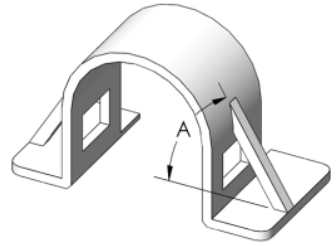

Solution 1: Increase rib angle

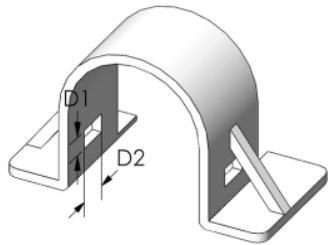

Solution 2: Change side cut dimensions

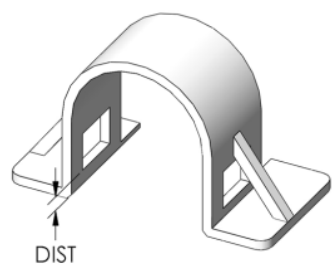

Solution 3. Move side cut down

Figure 31. Possible solutions to design problem 
The information included in the annotated model contains remarks that the original designer of the part considered valuable. This knowledge explicitly suggests maintaining the current rib angle and the dimensions of the side cut. These restrictions and design requirements will certainly determine how the model must be altered and what design conditions need to be met at all times. Therefore, according to the annotations, modifying the position of the side cuts (solution 3 ) is the most effective approach.

Based on this information, we performed a Chi-Square Test aimed at rejecting, with a level of significance $\alpha=0.05$, the null hypothesis $\mathrm{H}_{0 \text { (activity 2), }}$, defined as:

- $\mathrm{H}_{0(2)}$ : When critical design decisions about a model need to be made, annotated models are not more valuable than non-annotated models in terms of communicating design requirements.

- $\mathrm{H}_{\mathrm{a}(2)}$ : When critical design decisions about a model need to be made, annotated models are more valuable than non-annotated models in terms of communicating design requirements.

We define the modeling approach as the independent categorical variable $\mathrm{X}_{3}$ with values as shown in Table 18. The results of our study are shown in Table 19.

Table 18. Values of independent variable $\mathrm{X}_{3}$

\begin{tabular}{|cc|}
\multicolumn{1}{|c}{$\mathbf{X}_{3}$} & Description of the modeling approach \\
\hline Solution 1 & Increase rib angle \\
\hline Solution 2 & Change cut dimensions \\
\hline Solution 3 & Move side cut down \\
\hline Solution $4 *$ & Other approaches \\
\hline
\end{tabular}

* Includes incorrect approaches, such as adding new geometry to the cut as a new feature (inefficient), or trimming the size of the rib (incorrect as well, since the rib must remain at $5 \mathrm{~mm} \times 5 \mathrm{~mm})$ 
Annotation Mechanisms to Manage Design Knowledge in Complex Parametric Models and their Effects on Alteration and Reusability

Table 19. Observed values for experiment 2

Solution 1 Solution 2 Solution 3 Solution 4 Total

\begin{tabular}{|lccccr|}
\hline $\begin{array}{l}\text { Control Group: Non- } \\
\text { annotated model }\end{array}$ & 7 & 32 & 9 & 4 & 52 \\
\hline $\begin{array}{l}\text { Experimental Group: } \\
\text { Annotated model }\end{array}$ & 9 & 12 & 25 & 6 & 52 \\
\hline Total & 15 & 44 & 34 & 10 & 104 \\
\hline Chi-square=17.27, p-value<0.001 & & & & & \\
\hline
\end{tabular}

Based on the resulting $\mathrm{p}$-value $(\mathrm{p}<0.001)$, there is a statistically significant difference between the experimental and control groups. When critical design decisions about a model need to be made, annotated models are more valuable than non-annotated models in terms of communicating design requirements.

\section{Concluding remarks}

In this chapter, first steps towards understanding the potential of model annotations as design communication elements and evaluating user's response to different $\mathrm{CAD}$ challenges when design annotations are present were taken. The objective was to find evidence of higher quality modeling (in terms of methodology and reusability) when annotated models were used. Two experiments were conducted: a model alteration study and a design study. In both cases, results show that when users manipulate annotated models, even with no prior warning, they select more efficient modeling procedures and create models that are more reusable. Models that have been annotated with design intent information provide a statistically significant value over non-annotated models.

The number of potential hints that could unintentionally be given to users regarding subsequent modeling steps was minimized by providing a large number of annotations, most of them irrelevant to the task, in each model and by not giving any specific instructions about the existence or significance of the annotations. A similar study where participants are shown the initial and final versions of a more complex CAD model and asked to perform a longer sequence of alterations could provide further insights regarding the effects of annotations. This study could evaluate whether annotations have a significant impact on design intent communication (so users perform better), even when the geometry modifications are exposed at 
the beginning of the exercise. For a more comprehensive study, a larger and wider variety of models, representing a more diverse range of design scenarios, and a larger and more experienced sample of CAD users can be considered.

Although we consider this study a first approximation toward quantifying the full effects of model annotations, results suggest that users perform better in terms of modeling quality and reusability when design intent information is explicitly available as annotations. In the following chapter, a number of challenges related to the implementation of model annotations in practical environments are presented and the architecture of an annotation framework is introduced. 


\section{Chapter V \\ Extended Design Annotations}

\section{Introduction}

In this chapter, the challenges involved in implementing an annotation-based solution in production environments are discussed and a mechanism to communicate geometric design intent explicitly is presented. The mechanism consists in overloading and extending the scope of existing annotation instruments available in MBE environments. A new broader type of model annotation ("extended annotation") and the infrastructure required to support user interaction with the information are described. In order to manage the information stored in extended annotation structures effectively, a software module provides powerful filtering, editing, and visualization capabilities, giving users complete control of the information stored in the model.

Finally, the results of a study conducted with 60 participants to evaluate the performance of the proposed annotation model and the usability of the software module are presented.

\section{Implementation Challenges}

The effectiveness of model-based annotations is determined by the ability to clearly communicate information. Consequently, efficient visualization mechanisms become crucial, especially when users must handle heavily annotated models. While the use of annotated models as carriers of design 
knowledge has been discussed in previous chapters, it is necessary to examine the practical challenges involved and the limitations of current solutions. Five major challenges related to the practical implementation of annotations are discussed: storage, content, interface, visualization, and user motivation.

\subsection{Annotation Storage}

Annotations require efficient data structures to store information. These structures demand tools to store, visualize, and interact with the annotation content as well as instruments to manage the anchoring mechanism of the annotation. It is also necessary that representations are unified to make them platform-independent and avoid compatibility and portability issues.

As shown in the literature review chapter, annotations are classified as inline (internal), stand-off (external), and hybrid, based on how data is stored. Most PMI modules available in commercial CAD systems allow annotating models internally, although external annotations are more appropriate if the data needs to be shared. Since annotation information is kept separately, external annotations allow updates of the data without affecting the geometry of the model. Additionally, multiple annotation files can be linked to the same model to provide different annotated views to different users.

In terms of implementation, XML and SQL databases have been recognized as common data description standards. However, they are difficult to implement, partly because of the problem of persistent references, which describes the inconsistencies generated in the annotation structures when the geometry of the model being annotated changes or when there is a simultaneous writing access to the model from multiple users. Hybrid representation approaches have been proposed, where annotation information is stored both externally and internally within the model.

\subsection{Annotation Content}

An additional challenge regarding the implementation of annotated models involves the content structure, i.e. what information needs to be included and in what form, so information is communicated effectively. Naturally, decisions need to be made as to how design intent can be captured and communicated using annotations.

In order to provide computational support, design intent information must be represented in a structured manner. With a formal syntax, it is relatively simple for a computer to process and manage this information. However, 
fixed structures can also limit expressiveness and become intrusive to the user, which has in fact hindered the adoption of these tools in industry. For a designer, it is easier and more intuitive to use natural language, particularly because of the difficulty of representing heterogeneous information (such as design intent) with fixed structures. A recent approach proposed by [Sung et al., 2011] suggests logging the actions performed by a designer in a CAD session and interpreting patterns found in these actions, which minimizes user intervention in the process. Regardless of the technology, when users are allowed to use natural language, new challenges appear, such as minimizing the effects of writing style and language on communication effectiveness, determining the optimum annotation length so annotations are not ignored, and implementing natural language processing mechanisms so computational support can still be provided.

\subsection{Annotation Interface}

Methods to support interaction with annotations must allow users to enter and retrieve data easily and intuitively, as designers are often reluctant to spend additional time adding information to their models. The lack of adequate tools for knowledge-acquisition is in fact the major cause for the knowledge-acquisition bottleneck. Interface simplicity and integration with existing tools are crucial factors for the successful implementation of design annotations.

Although a number of prototypes have been developed [Boujut \& Dugdale, 2006; Sandberg \& Näsström, 2007], integration of the annotation tools with the modeling environment of the CAD application provides users with an already familiar interface, which minimizes the learning curve and the need to constantly switch between applications. In this context, Product and Manufacturing Information (PMI) modules available in modern CAD systems are already popular among engineers and designers so they are natural vehicles to interact with annotations.

\subsection{Annotation Visualization}

From a user interaction standpoint, an ever increasing number of annotations can quickly result in a cluttered model, which often creates confusion and a feeling of information overload in the user. In the area of information visualization, managing visual clutter is a crucial factor to ensure successful results. When too much data (or when data is not well organized) is displayed on a too small area, the value of the information diminishes. 
Although current model-based standards recommend the use of annotation groups or layers to simplify interaction, none of them provide specific guidelines to reduce visual clutter and thus, no practical implementations are available in current PMI modules. Advanced filtering and interactive navigation methods are needed as they are generally faster and do not rely on the user to create groups and distribute the annotations within these groups.

\subsection{User Motivation}

Most annotation and knowledge representation techniques have proven to be valuable. Nevertheless, they usually do not find acceptance in industry, as designers are reluctant to spend time annotating their designs. One reason is that the designer that has to implement the annotations has no further use of them, as she already understands the design. Why should the designer do something that is only beneficial for people that come after her? In many cases, she is missing incentives.

Convincing users to use annotations can clearly be a challenge, especially if the argument focuses exclusively on the collaborative aspect of helping other users. Even if the designer is forced to annotate her work, it is unclear that she will create quality annotations.

On the other hand, just as computer programmers comment their source code to document and recall specific changes and algorithms, product designers also need proper documentation to remember all the design changes and reasons for change of specific models. Therefore, automatic tools that kept a historical record of annotation information could motivate and incentivize designers. After all, historical annotation information may not just be valuable for future users of a model, but also for the original creators.

\section{Extended Annotations}

The continuous development of PMI modules by CAD software vendors and the support for current model-based definition standards open new opportunities for new types of annotations. In this context, one of the main contributions of this research is the introduction of extended annotations as bidirectional structures capable of carrying geometric design intent information both within 3D models and in an external repository.

We define an "extended annotation" as a 3D textual note with related metadata that is linked to a specific geometric feature of a 3D CAD model and associated to a certain information category such as geometric modeling 
Annotation Mechanisms to Manage Design Knowledge in Complex Parametric Models and their Effects on Alteration and Reusability

intent, product specification, or any other category defined by product lifecycle stakeholders. Extended annotation structures are natural extensions to the annotation mechanisms defined by current standards and implemented by modern CAD packages. In particular, the annotation system developed for this doctoral research involves the addition of new structures and connections to the 3D annotated model defined by the standard [SASIG, 2008] (see Figure 32).

The architecture of the proposed model is illustrated in Figure 33. Nodes in gray represent information stored outside the $3 \mathrm{D}$ model.

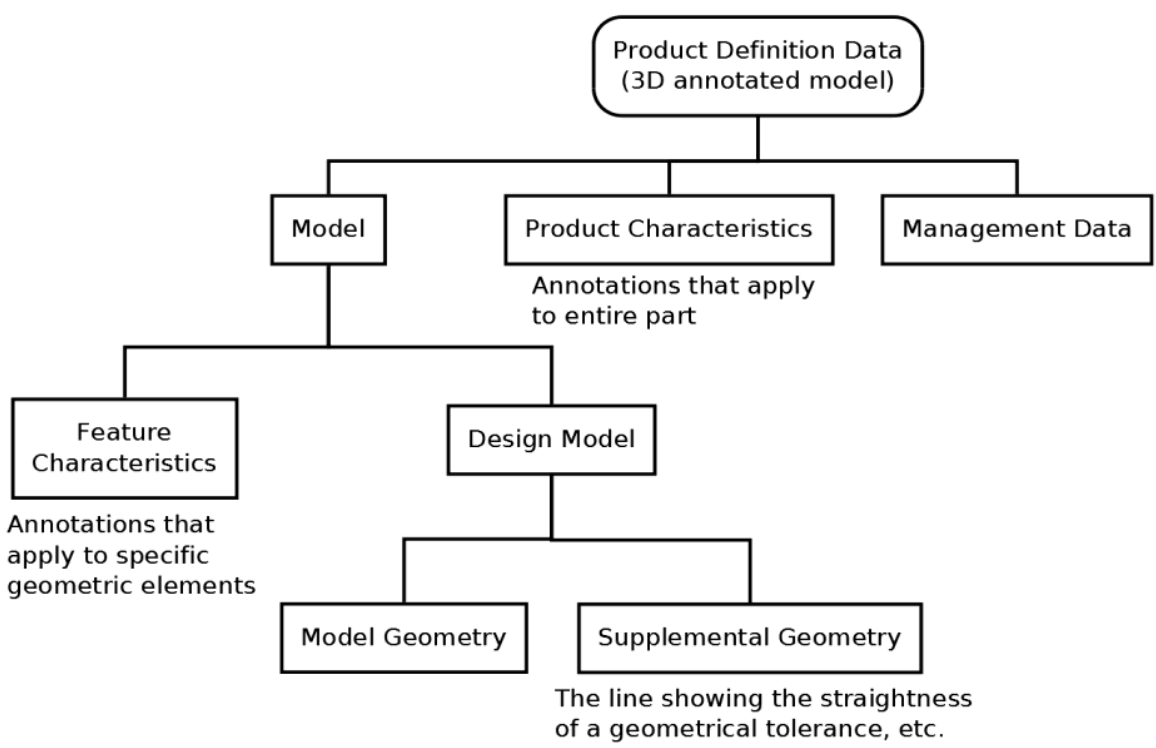

Figure 32. Information configuration of a 3D annotated model [SASIG, 2008] 


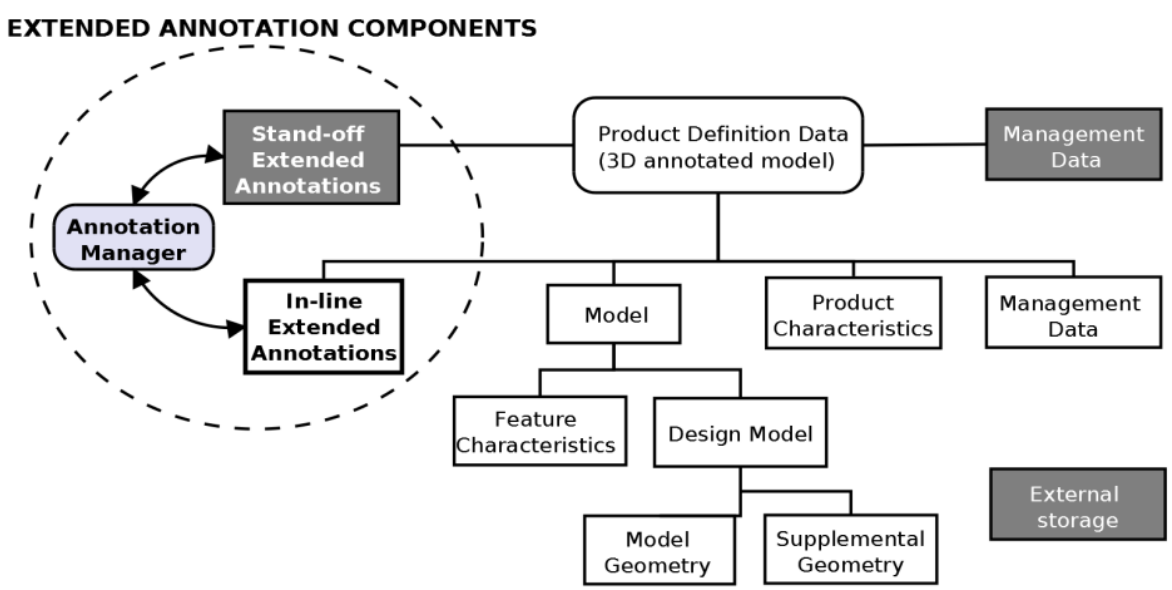

Figure 33. Proposed Extended Annotation Model (Adapted From [SASIG, 2008])

The proposed extended annotation model is comprised of three major components: the internal representation of the annotation, the extended external representation of the annotation, and a synchronization agent (or annotation manager) that ensures information integrity between the two representations (see Figure 34).

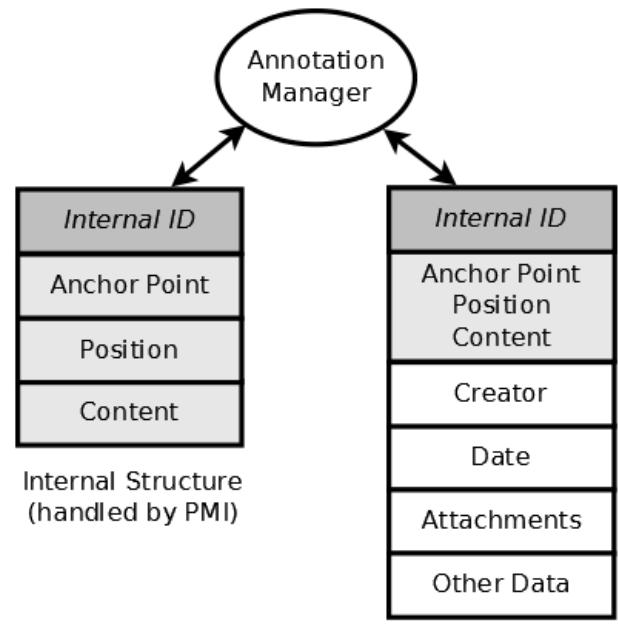

External Structure (handled by agent)

Figure 34. Components of Extended Annotation Model 
External representations are versions of the internal annotations that have been extended with additional data (creator, date, attachments, etc.) and are maintained in a separate repository. Therefore, the proposed approach transforms standard model annotations into extended annotations where information becomes more semantic and easier to manipulate.

Because of the limitations of the existing annotations structures available in PMI modules (visualization and interaction mechanisms such as filtering and searching are non-existent) a more flexible mechanism based on a dual representation is proposed. An additional contribution is a software module (called "annotation manager") that works as an automatic agent in charge of managing and synchronizing the dual representation of the annotations.

By allowing "in-line" techniques, a simple integration of the extended annotation model within existing CAD packages is ensured. Designers can thus annotate 3D models using already familiar tools, instead of spending valuable time learning new separate systems. This strategy minimizes the annotation workload and benefits from the familiarity of the users with existing software, which is a crucial factor for a practical implementation of the model and the avoidance of the knowledge-acquisition bottleneck. In the proposed model, the "in-line" aspects of the annotation are accomplished by overloading the functionality of the Product and Manufacturing Information (PMI) modules available in modern CAD systems.

The "stand-off" characteristics of extended annotations facilitate and optimize the visualization, search, and filtering of information. The visualization and display of 3D annotations are essential factors to ensure the effectiveness of an annotated model in terms of communication of information. In fact, the use of groups, layers, and annotation views to improve the readability of the annotations is specifically encouraged by the model-based standard, although no precise guidelines are provided regarding how this functionality should be implemented.

At this point, basic perception principles and its application to visual representations must be reviewed. The visual management of the annotations becomes especially relevant when the volume of the annotations in the 3D model grows to the point where it creates clutter and confusion (the problem of visual clutter reviewed in previous chapters), making the use of the annotated model impractical. The external representation of the extended annotation model along with the management capabilities offered by the annotation manager provides an automatic visualization framework for $3 \mathrm{D}$ 
annotations that frees the user from time-consuming tasks such as creating annotation views and organizing the information manually.

Furthermore, when the information is available outside the model, effective strategies can be developed to analyze the knowledge contained within the extended annotations. Additional information or metadata can be added to the external representation of the annotation (making it an "extended annotation") and synchronized with the internal version by the "annotation manager." By overloading the functionality of a typical PMI module, the "annotation manager" provides additional support for extended annotations.

In the following sections, the characteristics of the proposed model are described in terms of the annotation challenges discussed earlier in this chapter.

\subsection{Annotation Storage}

In the proposed model, 3D annotations are stored both internally within the CAD model, and externally. The internal representation of the annotation is managed directly by the PMI tools of the CAD system. The information includes the content of the annotation, the point of connection between the annotation and the aspect of the CAD model that is being annotated (typically, a face or a feature), and the identifier of the annotation element within the CAD file, used to uniquely identify the annotation and associate it with the corresponding external data. An extended annotation is defined externally as a set of textual elements, such as:

Extended_annotation $=\{$ Internal_ID, Type, Feature, Text, Creator, Date, [Additional_Items] \} where:

- Internal_ID is the unique identifier of the annotation element within the CAD model.

- Type is used to classify annotations into different categories, i.e. modeling annotations, manufacturing annotations, etc. It is intended for semantic searches.

- Feature is the specific geometric element or "form feature" of the CAD model that is being annotated.

- Text is the content of the annotation.

- Creator and Date represent the author of the annotation and the date of last modification. 
Annotation Mechanisms to Manage Design Knowledge in Complex Parametric Models and their Effects on Alteration and Reusability

- Additional_Items is an optional field that can be used to include other type of information such as hyperlinks or references to external documents that may be relevant to a specific part of the $3 \mathrm{D}$ model.

In terms of implementation, different types of external storage can be used to manage the external representation of the annotations, such as XML files or relational databases.

\subsubsection{XML Prototype}

An initial prototype was implemented using XML technology. A single XML file is used to store extended annotations of multiple 3D models that are related functionally, such as components of the same assembly, or models of the same family, such as different versions or variations of a particular CAD model.

Every annotation corresponds to a node in an XML tree, as shown in Figure 35. The textual data format and structured syntax of XML makes this language a suitable option for representing and accessing the specific elements of the extended annotations in an effective manner.

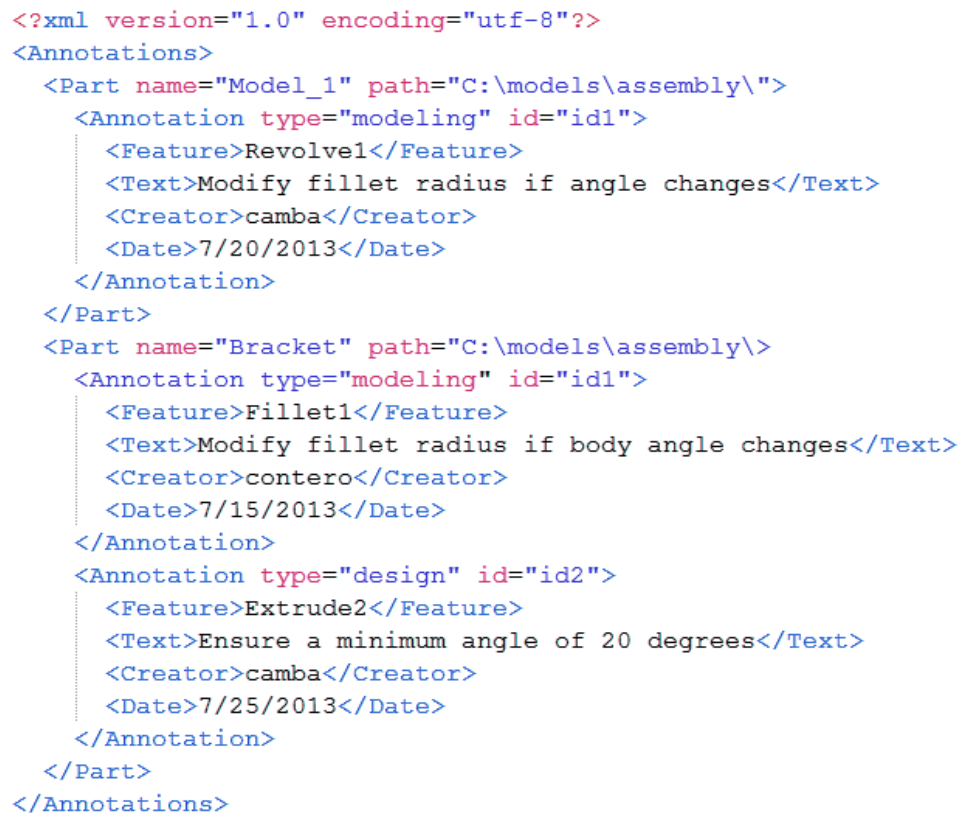

Figure 35. Structure of XML file 
The name of the 3D model and its file location are stored as attributes of the model node. To facilitate filtering and searching tasks, the child nodes "ID" and "type" can be converted to attributes of the corresponding parent node. The structure of the XML file is shown in Figure 36.

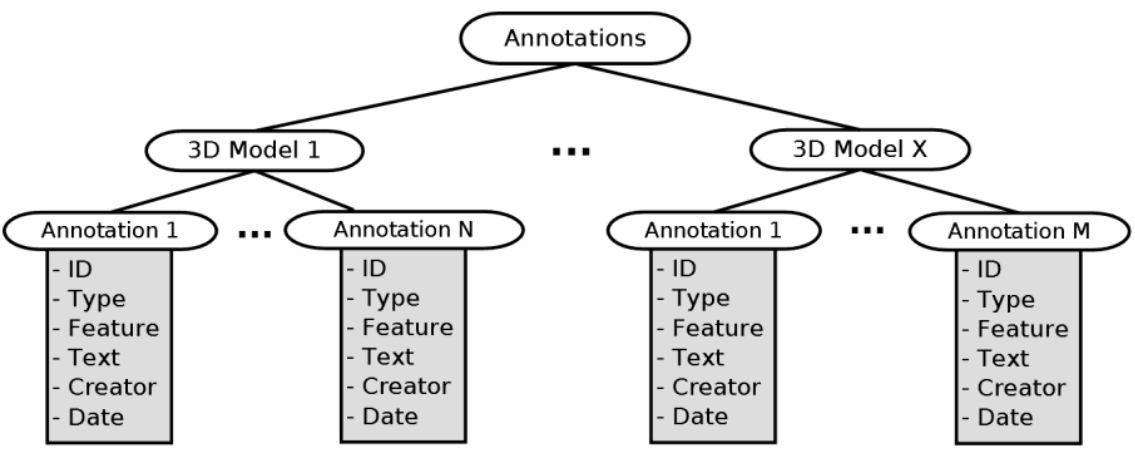

Figure 36. XML representation of extended annotations

\subsubsection{Relational Database Prototype}

As an alternative to the original XML representation, a second prototype was developed using a relational database. The availability of annotation information in a database facilitates the use of more powerful management, filtering, and searching mechanisms. This database implementation also facilitates support for other types of design knowledge such hyperlinks, sketches, graphical information, external documents, etc). The document management aspect of this functionality can be directly handled by the database system, whereas the links between external elements and annotations within the CAD model can be managed directly by the annotation manager. In addition, this architecture can be integrated within a Product Lifecycle Management (PLM) system, so the extended annotation system can be used more reliably in collaborative environments.

For the database implementation, an annotation table is defined where every child node of the original annotation node from the XML file becomes a field in the table. An additional field (model identifier) keeps a reference to the specific version of the $3 \mathrm{D}$ model the annotation is linked to. 
Annotation Mechanisms to Manage Design Knowledge in Complex Parametric Models and their Effects on Alteration and Reusability

The structure of this database implemented is described by the Enhanced Entity-Relationship (EER) diagram shown in Figure 37. The details of the PLM integration will be described in the next chapter.

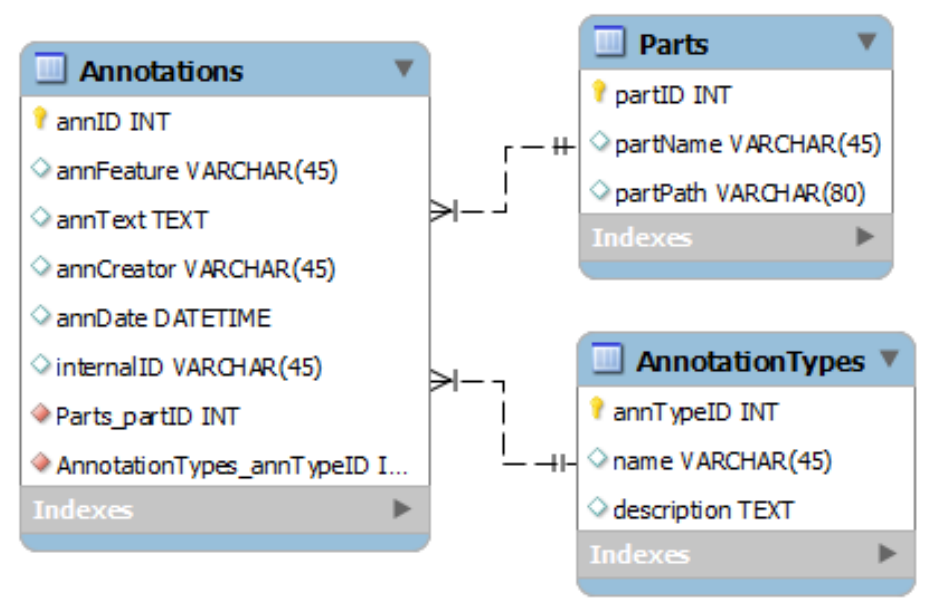

Figure 37. Enhanced Entity-Relationship (EER) database model for extended annotation system

\subsection{Annotation Content}

In order to support automated information processing, the proposed architecture offers support for structured annotation information. Although freestyle annotations are also supported, with a formal syntax it is relatively simple for a computer to process and manage annotation content.

To distinguish between annotation types (e.g. design intent, design rationale, etc.) labels or hash tags similar to those used by social networking sites are used. New annotation categories can be specified by defining new unique hash tags. For example, \#di can be used for design intent annotations, and \#dr for design rationale.

The structure of the extended annotation is completed by adding the author or creator of the annotation, the creation date, and the corresponding text explanation. The following are examples of annotations: 
- \#di@ johnsmith 04/23/13 apply symmetry copy function to build the part instead of creating a symmetric copy of the section in sketch.

- \#dr@peterhamilton 03/21/13 shell thickness increased 0.1" after FEM analysis detected high stress concentration in this area.

Annotations are stored internally within the CAD model using the previous structure. The different elements of the extended annotation are used externally to index, filter, and optimize searches. An annotated example of a 3D CAD model is shown in Figure 38.

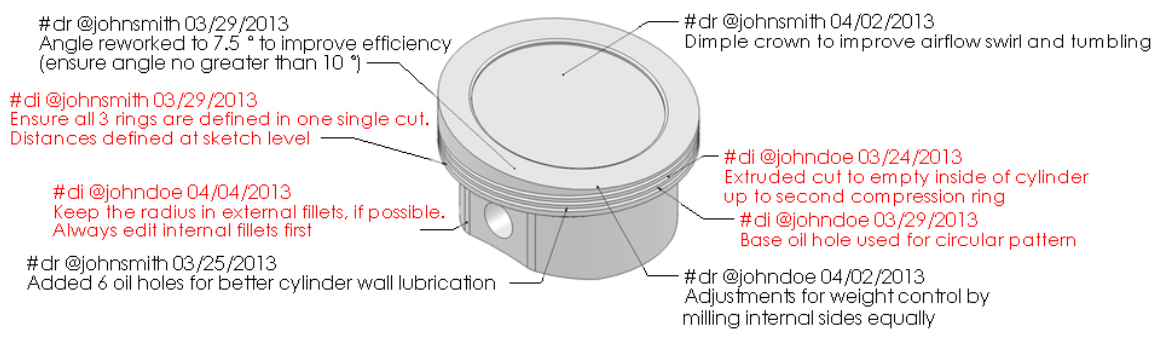

Figure 38. Annotated example

\subsection{Annotation Interface}

To provide an intuitive user interface to interact with extended annotations, a software module called "annotation manager" was developed. The annotation manager was originally conceived to explore the capabilities of current commercial CAD systems in terms of model annotation support. To ensure full integration with existing solid modeling applications, the prototype was implemented as a plug-in that can directly interact with the solid models created by these programs. DS SolidWorks ${ }^{\circledR}$ was selected due to availability reasons and the familiarity of the author with the SolidWorks ${ }^{\circledR}$ built-in Application Programming Interface (API) and development tools. Manipulation of model annotations is fully supported by the SolidWorks ${ }^{\circledR}$ API.

The annotation manager is a software component that keeps the internal and external representations of the model annotations synchronized, and provides a graphical user interface to interact with the information included in the extended annotations. In the extended annotation model, the annotation 
manager is envisioned as a fully integrated module of a traditional CAD system, possibly as part of the PMI toolset.

The creation and direct manipulation of the annotations (move, edit, delete, etc) within the 3D model, as well as the definition and selection of annotation planes, and visibility control are tasks that can be handled directly by standard PMI modules. Therefore, there is no need to duplicate this functionality in the annotation manager. Instead, the tool focuses on the synchronization, filtering, grouping, searching, and efficient visualization of information.

The synchronization of the internal annotations with the external repository (XML or database system) is handled by a background process that is triggered every time a new model annotation is created, modified, or deleted within the CAD package, or when the model is saved. No action is required from the user to maintain the information updated and synchronized. Likewise, internal annotations in the CAD model are automatically updated when changes are made to the external representations using the annotation manager, ensuring the bi-directionality of the extended annotation model. This background process also handles annotation change propagation to ensure consistency of changes by maintaining the most updated version of the annotations in both annotation structures. In case of inconsistencies, the process evaluates the information from the model with the information stored externally and prompts the user on how to proceed. Changes can be synchronized from the model to the external repository, from the external repository to the model, or they can be combined (updating both the external repository and the model) by comparing the annotations and maintaining only the most recent version in both places (see Figure 39).

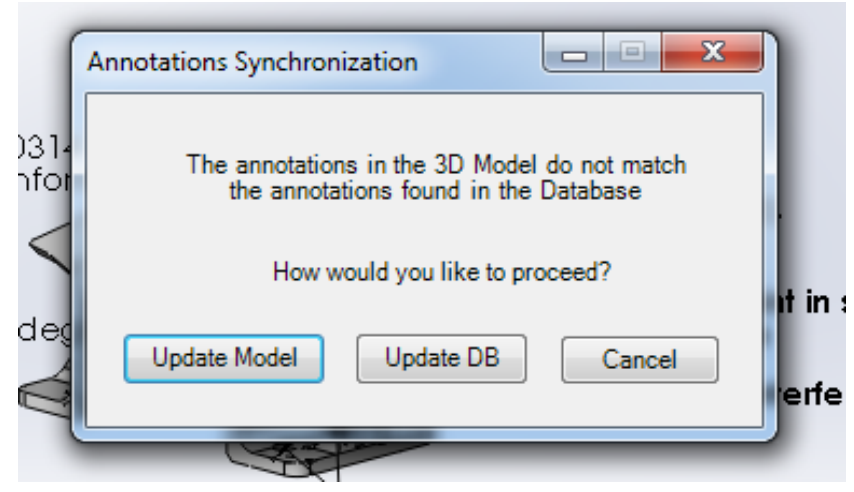

Figure 39. Annotation synchronization 
User interaction with the model annotations is achieved via a graphical interface that includes an annotation manager and the synchronized visualization screen, which is part of the CAD application (see Figure 40).

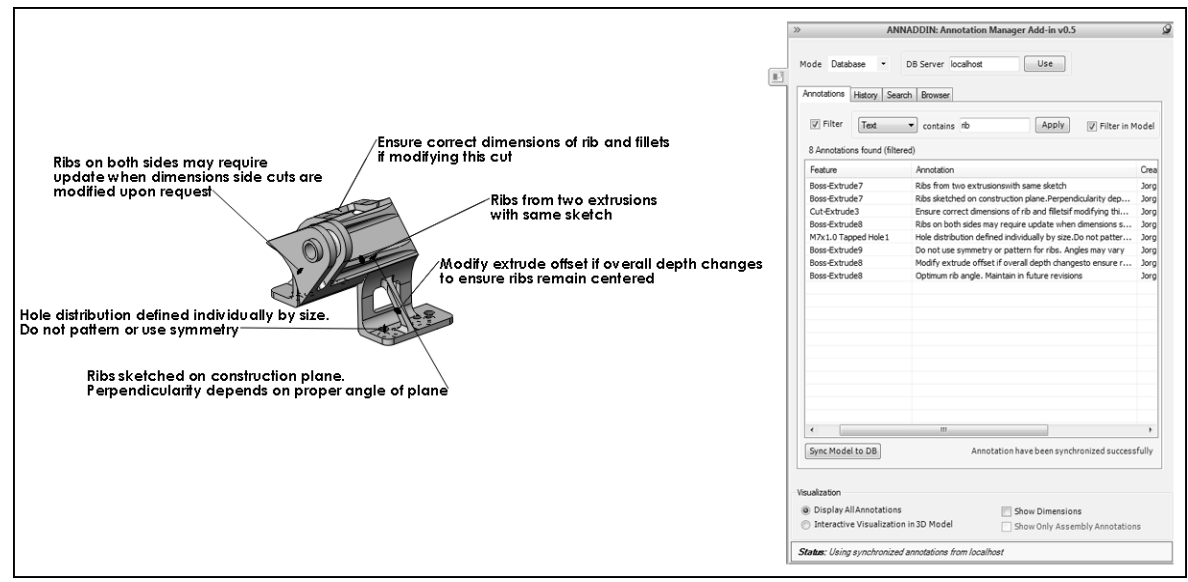

Figure 40. Example of annotated model (left) and software prototype (right)

The annotation manager keeps the most updated version of the annotation information on screen. The addition, modification, or deletion of an annotation has an immediate effect on the annotation manager's interface.

Taking advantage of the external representation of the annotation, the annotation manager provides tools to link annotations to other types of design knowledge such hyperlinks, sketches, graphical information, and external documents. The document management aspect of this functionality is handled by the database system, whereas the links between external elements and annotations within the CAD model are managed by the annotation manager. A browser-style document viewer fully integrated within the annotation manager allows users to link and examine documents and images related to the CAD model without ever leaving the CAD environment. In addition, searches and data mining techniques can be performed on external documents and immediately relate results to annotations and CAD models.

An example of an annotation linked to a HTML document and how the document is displayed by the annotation manager is shown in Figure 41. 


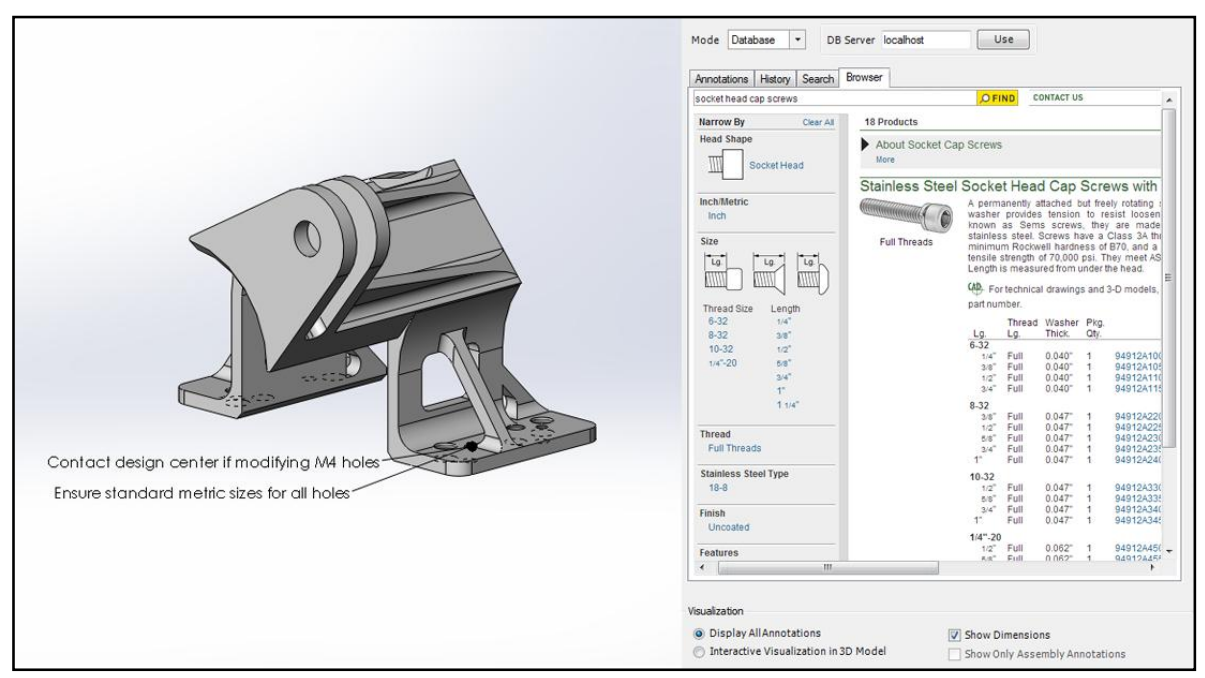

Figure 41. External HTML document linked to an annotation

\subsection{Annotation Visualization}

Filtering tools can contribute to reduce the amount of information on screen by showing or hiding specific model annotations based on user-defined criteria, such as date, feature, or a specific keyword. For example, users may select to only display the annotations associated to a particular surface of the model or created by a certain user.

An additional challenge regarding the use of model annotations, particularly the type of standard annotations provided by PMI modules in CAD environments, is related to their two-dimensional nature. Because annotations are essentially elements of plain text in 3D space, the user must use planes or views to host the annotations. As a result, some annotations may become visually unavailable when the user changes the viewpoint. Also, the creation and management of annotation planes and the distribution of annotations among these planes are ultimately the user's responsibility.

The annotation manager implements a graphical user interface to filter annotations based on different criteria. All model annotations are also displayed in the software interface in a tabular form, facilitating navigation through annotation information. When a filter is applied, annotations that are not relevant become automatically hidden both in the model and in the 
annotation list in the prototype (see Figure 40). Annotations become visible when the filter is reset or new criteria are defined.

Functionality for the interactive display of annotations based on the selection of model features in the design tree is also supported. This function automatically recognizes the anchor point of the annotation to the geometry, and identifies the surface with its corresponding modeling feature. This method automatically shows or hides annotations based on the feature that is currently selected in the model's design tree (see Figure 42).

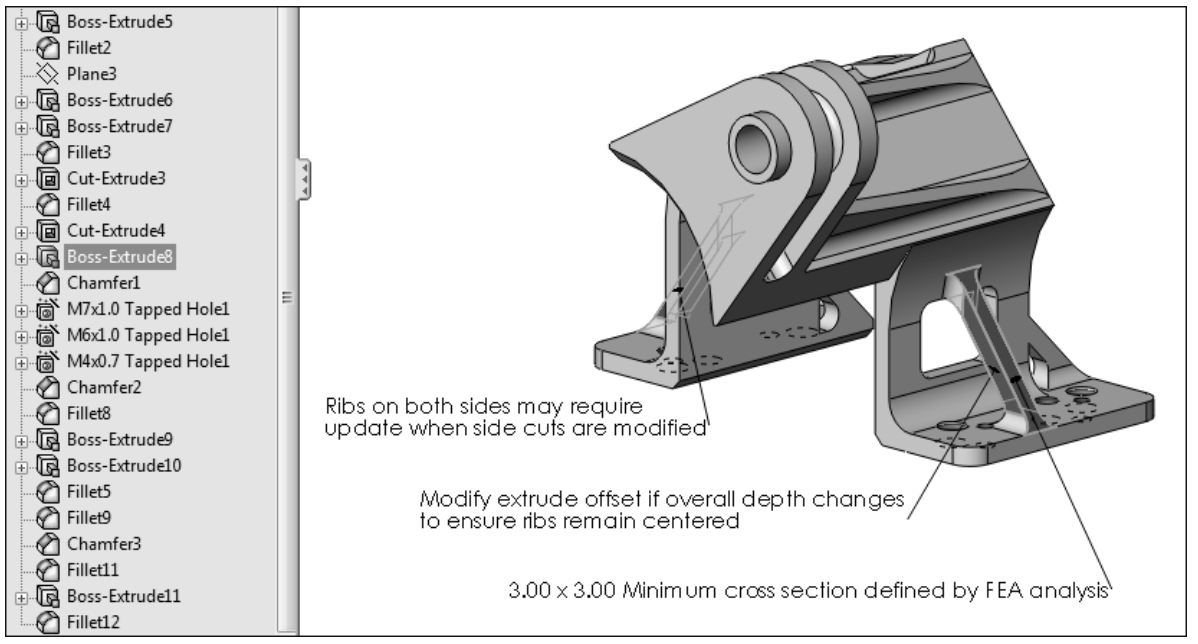

Figure 42. Example of interactive visualization of annotations. Only the 3D annotations connected to the selected feature (left) are displayed in the model (right)

\subsection{User Motivation}

As discussed earlier, most approaches to capture design intent do not find acceptance in industry because they typically require using additional complex tools that rely heavily on human involvement to interpret and load information. Designers are often missing incentives to enter and maintain this information in these systems.

One of the goals of the annotation manager is to provide a simple automated mechanism that is fully integrated within the CAD environment and allows designers to add relevant geometric information to a 3D model efficiently while maintaining a repository of design intent information. By overloading 
the functionality of a typical PMI module, the "annotation manager" provides a straightforward mechanism to manage annotation information that is built in the CAD interface and requires virtually no effort to learn. Designers do not need to leave the CAD environment and use separate software to annotate the models.

\section{Evaluation}

Three studies were conducted to test the effectiveness and user acceptance of the proposed model. The goal of the first study was determine the impact of the extended annotation model, particularly the annotation manager, in performing tasks that require finding information in an annotated 3D model. The second study, a pilot usability test, was performed to assess the application's user interface. Finally, a comparative study of different mechanisms to manage visual clutter in annotated 3D CAD models was performed to assess the value of the interaction tools provided by the annotation manager.

Two important characteristics of any system aimed at improving CAD model reuse and alteration are usability and efficacy. In terms of usability, authors [Drury et al., 2013] listed some of the more cited usability rules (e.g., be consistent) according to [Shneiderman \& Plaisant, 2009]; heuristics (e.g., make system status visible) based on [Nielsen \& Mack, 1994]; and principles (e.g., provide feedback) according to [Norman, 2002], for human and computer interaction. In this work, usability is assessed by using an adapted version of the questionnaire developed by [Chin et al., 1988]. The efficacy of our system is assessed in a similar manner to the work by [Lenne et al., 2009] by determining the time required for participants to find the correct answer to design questions both with and without the annotation manager system.

Because of the difficulty of evaluating an experimental tool in a real industrial environment (personnel availability, daily workload and deadlines of designers, integration risks, and time involved in familiarize users with the new system, etc), the system was tested in an academic setting.

\subsection{Experiments 1 and 2: User performance}

A total of 60 volunteers (students and faculty) participated in the study and evaluated the tool's usability. All participants had previous technical training in engineering design graphics and with SolidWorks ${ }^{\circledR}$ and/or other CAD packages. 
The experiments were conducted in a computer laboratory environment where participants were called one at a time to complete the exercises. This procedure helped prevent involuntary peer pressure on participants that took longer times to finish. Participants were equipped with a computer with two monitors: the first one showing the CAD package (SolidWorks ${ }^{\circledR}$ ) with the annotated 3D model required for the exercises, and the second showing the annotation manager prototype. A total of 30 annotations were included in the model. All dimensions were made visible as well.

Proper feature naming conventions were not considered in the model used for this study. Features of the model used in the study show default names with sequential number indicating the order of creation. Although proper naming practices can add a certain level of expressive value to the model, its relevance in this study is limited. Due to the nature of the design intent information used in the annotations (e.g. "Ensure a minimum angle of 20 degrees" or "Thickness increased after FEA results", etc.), the use of representative feature names is not practical as they cannot efficiently communicate the message. Therefore, their effect in our experiment is negligible.

Two sets of two exercises were developed and given to participants (see Appendix C). The objective of the exercises in the first set is to find a specific model annotation with the answer to a question. The exercises in the second set are design problems where certain modifications need to be performed to the $3 \mathrm{D}$ model. These alterations intentionally cause rebuild errors or unwanted effects in other parts of the model, which can be resolved by taking different approaches. Five possible answers (in multiple-choice format) were presented to the participants, who were asked to select the correct answer based on the annotations defined in the model.

All participants answered all four questions, but were only asked to use the annotation manager for one question from the first group and one question from the second group. As a result, all participants contributed to both the control and experimental groups. All the questions used in the study are available in Appendix C. Initially, participants were given a brief presentation with a demonstration of the software prototype, and were allowed a few minutes to familiarize themselves with the 3D model and the software. Questions were given one at a time, and participants' activities were timed (starting from the time they received the question) to determine how long they required to find the correct answer. 

their Effects on Alteration and Reusability

A t-test was used to examine the difference in the time required to complete the four design questions with and without the annotation manager. For each question, the mean time required to answer the question was significantly less with the aid of the extended annotation system than without. These results (see Table 20) show a statistically significant benefit of using the extended annotation system for all four questions.

Table 20. T-test comparison of time (s) required to answer questions (n: sample size, $\mathrm{M}$ : time in seconds)

\begin{tabular}{|ccccccc|}
\hline & \multicolumn{2}{c}{$\begin{array}{c}\text { With ann. } \\
\text { manager }\end{array}$} & \multicolumn{2}{c|}{$\begin{array}{c}\text { Without ann. } \\
\text { manager }\end{array}$} & & \\
\hline Question & $n$ & $M$ & $n$ & $M$ & $t$ & $p$ \\
\hline$\# 1$ & 30 & 65.5 & 30 & 212.6 & 11.10 & $<0.001$ \\
\hline$\# \mathbf{2}$ & 30 & 72.5 & 30 & 131.3 & 12.91 & $<0.001$ \\
\hline$\# 3$ & 30 & 169.4 & 30 & 310.2 & 14.83 & $<0.001$ \\
\hline$\# 4$ & 30 & 370.9 & 30 & 663.1 & 15.16 & $<0.001$ \\
\hline
\end{tabular}

As a second study, a psychometrically validated usability questionnaire adapted from [Chin et al., 1988] was distributed to the participants at the end of the session. A set of questions were presented using a numerical ten-point Likert scale. The questions were intended to evaluate the usability of the annotation manager and the user satisfaction levels with the tool. These levels are measured using the mean and standard deviation of the data obtained from the participants' responses. The questions given to the participants as well as the statistical measures used to analyze the results are shown in Table 21. No responses were ranked below 5.

In general, the functionality of the annotation manager was well received, with most participants expressing positive reactions, acknowledging its value, and ranking the application highly in most areas. It is important to note the particularly high scores given to questions A2: "Usefulness and value of the application" and B3: "Highlighting of annotations on the 3D model simplifies tasks." The positive evaluation results shown in Table 21 are especially relevant when viewed in combination with the significant improvement in terms of the time required to find information for completing a task, as reported above and illustrated in Figure 43. 
Table 21. User Satisfaction Questionnaire and Results

\begin{tabular}{|c|c|c|c|c|}
\hline \multicolumn{2}{|c|}{$\begin{array}{l}\text { Question } \\
\text { Overall Reactions to the Software }\end{array}$} & Scale & Mean & Std. Dev. \\
\hline A1 & Overall experience with the software & 0 (terrible) - 9 (wonderful) & 7.03 & 0.99 \\
\hline A2 & $\begin{array}{l}\text { Usefulness and value of the } \\
\text { application }\end{array}$ & $\begin{array}{l}0 \text { (not useful or valuable) }-9 \\
\text { (very useful and valuable) }\end{array}$ & 7.72 & 0.83 \\
\hline A3 & Ease of Use & 0 (difficult) - 9 (easy) & 7.95 & 0.79 \\
\hline A4 & $\begin{array}{l}\text { Level of satisfaction with the } \\
\text { software }\end{array}$ & 0 (frustrating) - 9 (satisfying) & 7.63 & 1.02 \\
\hline A5 & $\begin{array}{l}\text { Level of interest and motivation to } \\
\text { use the software }\end{array}$ & 0 (dull) - 9 (stimulating) & 7.22 & 1.04 \\
\hline \multicolumn{5}{|c|}{ Screen } \\
\hline B1 & $\begin{array}{l}\text { Organization of information on } \\
\text { screen }\end{array}$ & 0 (confusing) - 9 (very clear) & 7.88 & 0.80 \\
\hline B2 & $\begin{array}{l}\text { Visualization of annotations in the } \\
\text { application }\end{array}$ & $\begin{array}{l}0 \text { (unclear \& hard to read) - } 9 \\
\text { (clear and easy to read) }\end{array}$ & 8.15 & 0.82 \\
\hline B3 & $\begin{array}{l}\text { Highlighting of annotations on 3D } \\
\text { simplifies tasks }\end{array}$ & 0 (not at all) - 9 (very much) & 8.52 & 0.57 \\
\hline \multicolumn{5}{|c|}{ Terminology and System Information } \\
\hline $\mathrm{C} 1$ & Use of terms throughout system & $\begin{array}{l}0 \text { (inconsistent) }-9 \\
\text { (consistent) }\end{array}$ & 8.12 & 0.67 \\
\hline $\mathrm{C} 2$ & $\begin{array}{l}\text { Messages on screen which prompt } \\
\text { user for input }\end{array}$ & 0 (confusing) - 9 (clear) & 7.45 & 1.02 \\
\hline $\mathrm{C} 3$ & $\begin{array}{l}\text { Computer keeps you informed about } \\
\text { what it is doing }\end{array}$ & 0 (never) - 9 (always) & 6.85 & 0.92 \\
\hline \multicolumn{5}{|c|}{ Learning } \\
\hline D1 & Learning to operate the system & 0 (difficult) - 9 (easy) & 8.43 & 0.59 \\
\hline D2 & $\begin{array}{l}\text { Exploring new features by trial and } \\
\text { error }\end{array}$ & 0 (difficult) - 9 (easy) & 7.17 & 0.99 \\
\hline D3 & $\begin{array}{l}\text { Remembering names and use of } \\
\text { commands }\end{array}$ & 0 (difficult) - 9 (easy) & 8.20 & 0.78 \\
\hline D4 & $\begin{array}{l}\text { Tasks can be performed in a straight- } \\
\text { forward manner }\end{array}$ & 0 (never) - 9 (always) & 7.95 & 0.79 \\
\hline \multicolumn{5}{|c|}{ System Capabilities } \\
\hline E1 & System speed & 0 (too slow) - 9 (fast) & 8.18 & 0.83 \\
\hline E2 & System reliability & 0 (unreliable) - 9 (reliable) & 7.83 & 0.98 \\
\hline E3 & Correcting your mistakes & 0 (difficult) - 9 (easy) & 7.75 & 1.02 \\
\hline
\end{tabular}


Annotation Mechanisms to Manage Design Knowledge in Complex Parametric Models and their Effects on Alteration and Reusability

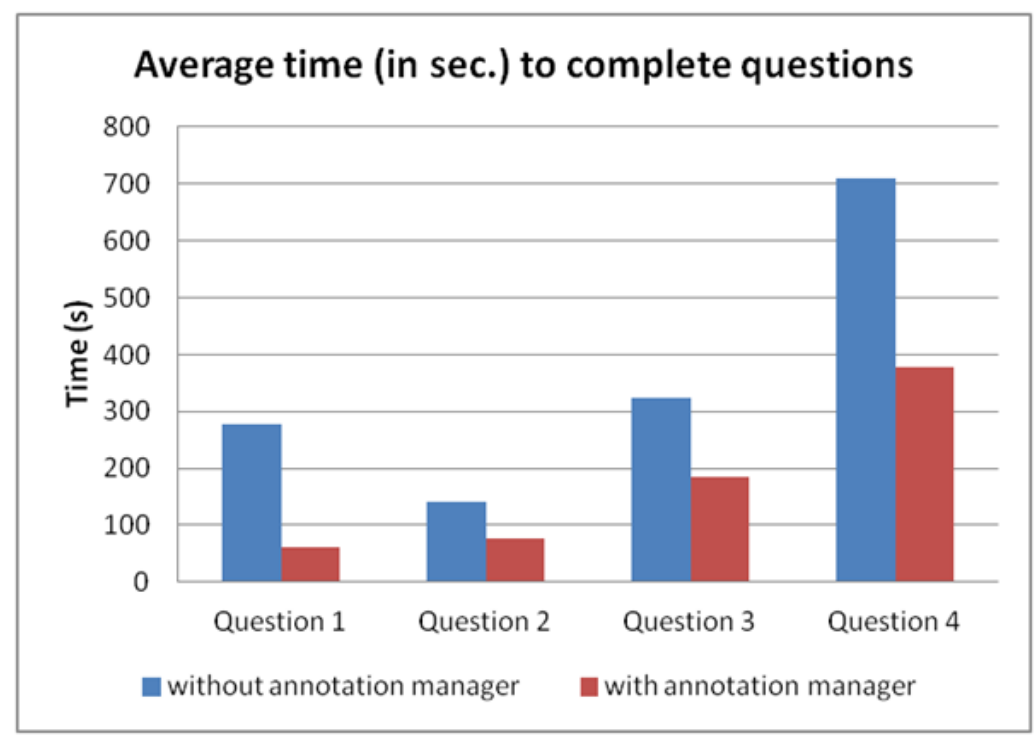

Figure 43. Average time improvements with annotation manager

Observation of the participants' behavior and their approaches to the problems presented confirms our initial assumption: visual clutter is an important problem when the user has to interact with an extensively annotated model. While organizing annotations in views is critical for regular 3D annotations (as suggested by the SASIG standard), implementing the extended annotation model is not practical without a tool like the annotation manager, as visual clutter would prevent exploiting all the benefits that the explicit communication of design intent represents for model alteration and reuse.

\subsection{Experiment 3: Visual Clutter}

For this study, a total of 120 participants (divided into four groups of thirty) in a CAD laboratory environment were given an annotated model and asked to find specific model annotations with the answers to four questions. Questions were presented one at a time. The first two questions (short answer) required participants to write their answer based on the information found in one particular annotation. The remaining two questions were presented in a multiple choice format with four possible answers. They were formulated as design problems that required performing minor modification to the 3D model, but intentionally caused rebuild errors and unwanted 
effects in certain parts of the geometry. All possible answers described an adequate modeling approach to solve the design problem. Participants were asked to select the correct answer based on the information found in a specific group of model annotations. The time employed by participants to find the correct answer was recorded (starting from the time they received the question).

A CAD model with a total of thirty annotations deliberately arranged to create visual clutter was given to the participants. All dimensions were also visible. The content of the annotations ranged from design and manufacturing information (i.e. Refer to model AA0314 for weight control information, Dimensional adjustments for weight control must be made in unmachined areas) to design intent and modeling procedures (i.e. Modify extrude offset if overall depth changes to ensure ribs remain centered, Hole distribution defined individually by size. Do not pattern or use symmetry). The annotations that are relevant to the questions of the study are shown in Table 22. In order to supplement instructions and clear any possible doubts, all questions were accompanied by illustrations of the 3D model, with specific areas highlighted. Such illustrations have been omitted in Table 22 for clarity.

Each group was randomly assigned an annotation mechanism, as shown in Table 23. Participants in group 3 were given a brief demonstration of the plug-in and were allowed a few minutes to familiarize themselves with the software. For participants in group 4, the prototype was preset to work in interactive mode. Therefore, there was no need to provide additional instructions.

For participants in group 2, we decided to create six annotation groups and distribute the annotations among these groups. Groups were based on the following criteria: annotations that communicate restrictions to the model (i.e. Ensure standard metric sizes for all holes), previous changes performed to the model (i.e. Thickness increased after initial FEA test results), warnings about modeling actions (i.e. Hole distribution defined individually by size. Do not pattern or use symmetry), manufacturing notes (i.e. Break all sharp edges to R4), optional elements (i.e. Optional for weight control when required), and modeling decisions (i.e. Ribs sketched on construction plane. Perpendicularity depends on proper angle of plane). The time employed by our team to define and create the annotation groups in the model was not considered in our statistical analysis, although it clearly has a significant effect on efficiency. 
Annotation Mechanisms to Manage Design Knowledge in Complex Parametric Models and their Effects on Alteration and Reusability

Table 22. Questions and relevant annotations with explicit information to answer correctly

Question

Answer Annotations

1 What should you before modifying the size of the M4 machine holes?

Contact Design Contact Design Center Center if modifying M4 holes.

2 What is the range of acceptable angles for the $\operatorname{Min}=20^{\circ}$ front face of the part?

$\operatorname{Max}=27^{\circ}$

Ensure min angle of 20 degrees, Ensure max angle of 27 degrees

3 What is the most effective procedure to create a second rib on the model?

b)

Do not use symmetry or a) Mirror the first rib. pattern for ribs. Angles

b) New sketch on surface and extrude.

c) Linear pattern using the original rib.

d) Use existing sketch of original rib to create new extrusion at offset distance.

4 When modifying the dimensions of the ribs, errors occur in the model. What is the procedure to solve these errors?

b) may vary.
a) Reduce the height of the cut.
b) Move the cut down.
c) Increase the angle of the ribs.
d) The dimensions of the side ribs cannot be changed.

Optimum rib angle.
Maintain in future
versions.
$3.00 \times 3.00$ Minimum
cross section defined by
FEA analysis.
Keep dimensions of the
cut constant. Modify
position if necessary.

Table 23. Annotation mechanisms and descriptions

\begin{tabular}{|clll|}
\hline $\mathbf{1}$ & $\begin{array}{l}\text { All annotations } \\
\text { on screen }\end{array}$ & No annotation management tools & Tool \\
\hline $\mathbf{2}$ & $\begin{array}{l}\text { Layers and } \\
\text { Grouping }\end{array}$ & $\begin{array}{l}\text { Annotations manually organized in groups } \\
\text { by a member of the research team }\end{array}$ & $\begin{array}{l}\text { PMI module: standard } \\
\text { annotation views }\end{array}$ \\
\hline $\mathbf{3}$ & Filtering & $\begin{array}{l}\text { Annotations can be filtered based on } \\
\text { different criteria: content, feature, etc. }\end{array}$ & $\begin{array}{l}\text { Custom plug-in } \\
\text { developed in-house }\end{array}$ \\
\hline $\mathbf{4}$ & $\begin{array}{l}\text { Model's Design } \\
\text { Tree }\end{array}$ & $\begin{array}{l}\text { Interactive visualization using the features } \\
\text { available in the model's design tree. }\end{array}$ & $\begin{array}{l}\text { Custom plug-in } \\
\text { developed in-house }\end{array}$ \\
\hline
\end{tabular}


To statistically evaluate the effect of the various annotation mechanisms, the mean time to correctly answer each question in the various mechanism groups were compared using a two sample t-test. These mean times are shown in Table 24 along with the standard deviation for each group and question. Group 1, with all annotations shown, was designated the control group; the mean answer time for each of the questions (and the total) for each other group was compared to group 1 . These results are also shown in Table 24.

For all four questions and all three groups, the time required to correctly answer the question was statistically significantly lower $(a=0.05)$ than that of group 1. For all four questions, the time required was the least for group 4.

Table 24. Question completion time by group and statistical comparison to Group 1

\begin{tabular}{|cccccccc|} 
& Group 1 & \multicolumn{2}{c}{ Group2 } & \multicolumn{2}{c}{ Group 3 } & \multicolumn{2}{c|}{ Group 4} \\
& $M$ & $M$ & $t$ & $M$ & $t$ & $M$ & $t$ \\
& $(S D)$ & $(S D)$ & $p$ & $(S D)$ & $p$ & $(S D)$ & $p$ \\
\hline \multirow{2}{*}{ Question 1 } & 212.6 & 82.2 & 9.89 & 65.5 & 11.10 & 54.0 & 12.10 \\
& $(70.4)$ & $(16.0)$ & $<0.001$ & $(17.7)$ & $<0.001$ & $(14.0)$ & $<0.001$ \\
\hline \multirow{2}{*}{ Question 2 } & 131.3 & 77.0 & 12.48 & 72.5 & 12.91 & 69.5 & 13.36 \\
& $(77.0)$ & $(13.3)$ & $<0.001$ & $(15.2)$ & $<0.001$ & $(15.8)$ & $<0.001$ \\
\hline \multirow{2}{*}{ Question 3 } & 310.2 & 168.7 & 21.90 & 169.4 & 14.83 & 153.3 & 21.17 \\
& $(168.7)$ & $(26.5)$ & $<0.001$ & $(46.4)$ & $<0.001$ & $(33.1)$ & $<0.001$ \\
\hline \multirow{2}{*}{ Question 4 } & 663.1 & 363.8 & 16.68 & 370.9 & 15.16 & 320.6 & 18.39 \\
& $(362.8)$ & $(30.1)$ & $<0.001$ & $(48.4)$ & $<0.001$ & $(40.0)$ & $<0.001$ \\
\hline \multirow{2}{*}{ Total } & 1317.1 & 690.7 & 21.71 & 678.3 & 21.02 & 597.4 & 23.83 \\
& $(690.7)$ & $(50.2)$ & $<0.001$ & $(72.4)$ & $<0.001$ & $(70.1)$ & $<0.001$ \\
\hline
\end{tabular}

The total time required to correctly answer all for questions was compared for each of the alternative annotation mechanisms. These results are shown in Table 25 . There was no statistically significant difference in the total time required to answer the questions between the mechanisms used for groups 2 and 3. Only Question 1 showed a statistically significant difference between groups 2 and $3(\mathrm{t}=3.83 ; \mathrm{p}=<0.001)$. In the comparison between groups 2 and 4, only Question 3 did not show a statistically significant difference 
$(\mathrm{t}=1.99 ; \mathrm{p}=0.052)$. In the comparison of groups 3 and 4 , Questions 2 $(\mathrm{t}=0.76 ; \mathrm{p}=0.447)$ and $3(\mathrm{t}=1.54 ; \mathrm{p}=0.128)$ did not show statistically significant differences between the two mechanisms.

Although group 4 was the best performer in terms of time to find specific annotations, it cannot be concluded that the interaction mechanism used by this group is the most efficient in terms of communicating information. In a situation where one or more features in the design tree are left unexplored, the potential annotations connected to them will never be displayed. Therefore, some relevant information may never reach the user. Nevertheless, interactive visualization via the design tree has proven successful in reducing visual clutter on screen.

Table 25. Total time for all questions data comparison

\begin{tabular}{|cccc|} 
& Group 2 & Group 3 & Group 4 \\
& $t$ & $t$ & $t$ \\
& $p$ & $p$ & $p$ \\
\hline Group 1 & 21.71 & 21.02 & 23.83 \\
& $<0.001$ & $<0.001$ & $<0.001$ \\
\hline Group 2 & & 0.77 & 5.93 \\
& & 0.444 & $<0.001$ \\
\hline Group 3 & & & 4.40 \\
& & & $<0.001$ \\
\hline
\end{tabular}

We observed a tendency in a large number of participants in group 4 (interaction using the design tree) to click and select surfaces directly in the 3D model (as opposed to selecting the feature in the design tree) to try to activate the associated annotations. When only one surface of the model is selected, the current version of the software prototype will only display the annotations that are directly anchored to that surface. However, when a feature in the design tree is selected, the tool will show all the annotations connected to any of the surfaces in that feature. In our experiments, a number of participants did not realize this behavior until after several trials. These actions could explain some of the slower times recorded for this group, particularly in the first activity. On the positive side, these results can also be interpreted as indicators of a more intuitive behavior expected by the user, which can be an important factor to consider in future implementations of the prototype. 


\section{Concluding Remarks}

In this chapter, the limitations of current annotation models were exposed; specifically, highlighting the limitations placed on users in light of numerous annotations. A broader and more interactive structure was introduced and the feasibility of this new model in terms of design intent communication was demonstrated. The preliminary studies conducted with the software prototype reveal the value of the annotations as carriers of design information when proper managing, filtering, and visualization mechanisms are in place.

The results of the three studies conducted with the annotation manager expose the problem of managing visual clutter created by 3D annotations (even in models with a relatively small number of annotations) and confirm the need for intuitive methods to browse and filter annotations. As originally anticipated, users that were asked to retrieve information from models with no visualization or filtering tools performed statistically significantly worse than users with access to annotation management mechanisms. On the other hand, filtering and interactive navigation based on the model's design tree are faster and more efficient techniques than organizing the annotations in groups or annotation views (as suggested by current standards), especially if time required to create the groups and properly distribute the annotations within these groups are taken into consideration.

The current version of the annotation manager prototype allows users to manually select the annotations to display, but only differentiates between two groups of information: selected annotations (highlighted) and unselected annotations. More sophisticated grouping strategies should be explored in the next version of the tool. First, further capabilities of color should be explored to differentiate more than two groups by graying out visible annotations that are unrelated to the selected ones, thus creating three groups: selected, related, and nonrelated. This strategy is intended to support users during visual searches. In heavily annotated models, other perceptual grouping strategies, such as size and patterns, can also be explored to assist users in browsing the related annotations.

The use of the proposed extended annotations encourages the definition of more comprehensive CAD models, where annotation information can be used as a resource for data analysis techniques. For example, different characteristics of the annotations can be analyzed, potentially inspiring techniques and quality metrics for improving CAD model reuse, or as a basis to collaboratively define sets of best design and modeling practices. 


\section{Chapter VI Integration in Collaborative Environments}

\section{Introduction}

In this chapter, a further exploration of the extended annotation model and three practical applications are presented. First, an integration architecture where synchronized extended versions of model annotations can be maintained and incorporated into a Product Lifecycle Management (PLM) system is described. Next, an annotation history module, also integrated with the PLM system, is described as a tool to keep track of the changes and actions performed to the model annotations.

Finally, we incorporated communication functionalities to the extended annotation concept by using an interactive mechanism based on video conferencing that enables users to quickly communicate with the model annotators in situations where the contents of the annotation need to be extended or clarified. This approach eliminates the need for separate communication tools and provides a collaborative space that is built directly into the CAD interface.

\section{Product Lifecycle Management Systems}

Product Lifecycle Management (PLM) refers to the activity of managing all product related information and processes across the enterprise throughout its entire lifecycle, from concept to retirement. It is a concept that integrates 
information, people, processes and business systems, providing a product information backbone for the extended enterprise.

As a technology solution, it establishes a set of tools and technologies that provide a shared platform for collaboration among product stakeholders and streamlines the flow of information along all the stages of the product life cycle [Ameri \& Dutta, 2005].

For an enterprise, the advantages of adopting a PLM system are numerous, including more efficient processes related to New Product Development (NPD) activities, increased innovation and faster response times to market changes, reduced project failure rates, faster delivery, more effective communication among teams, and minimized manufacturing costs.

PLM is broad concept typically connected to other business technologies such as Customer Relationship Management (CRM), Supply Chain Management (SCM), and Enterprise Resource Planning (ERP). These four technologies are often considered the basis of a company's information technology infrastructure. In the context of the collaborative engineering paradigm, the role and components of a PLM system are illustrated in Figure 44.

PLM is comprised of four functional blocks:

- Product definition and related processes:

CAD/CAM/CAE/CAPP/CAT, digital mock-up, virtual prototyping, digital manufacturing.

- Computer Supported Collaborative Work (CSCW): Videoconferencing, Electronic blackboards, etc.

- Coordination tools: Workflow, project management and project planning

- Product Data Management (PDM): Vaulting, role definition, versioning, data lifecycle management.

The PDM component is the system in charge of tracking and managing the information generated during the product lifecycle with the purpose of improving productivity and reusability of product data and enhancing collaboration. This information includes CAD models, assemblies, finite element analysis, engineering change management information, engineering drawings, component libraries, etc. 


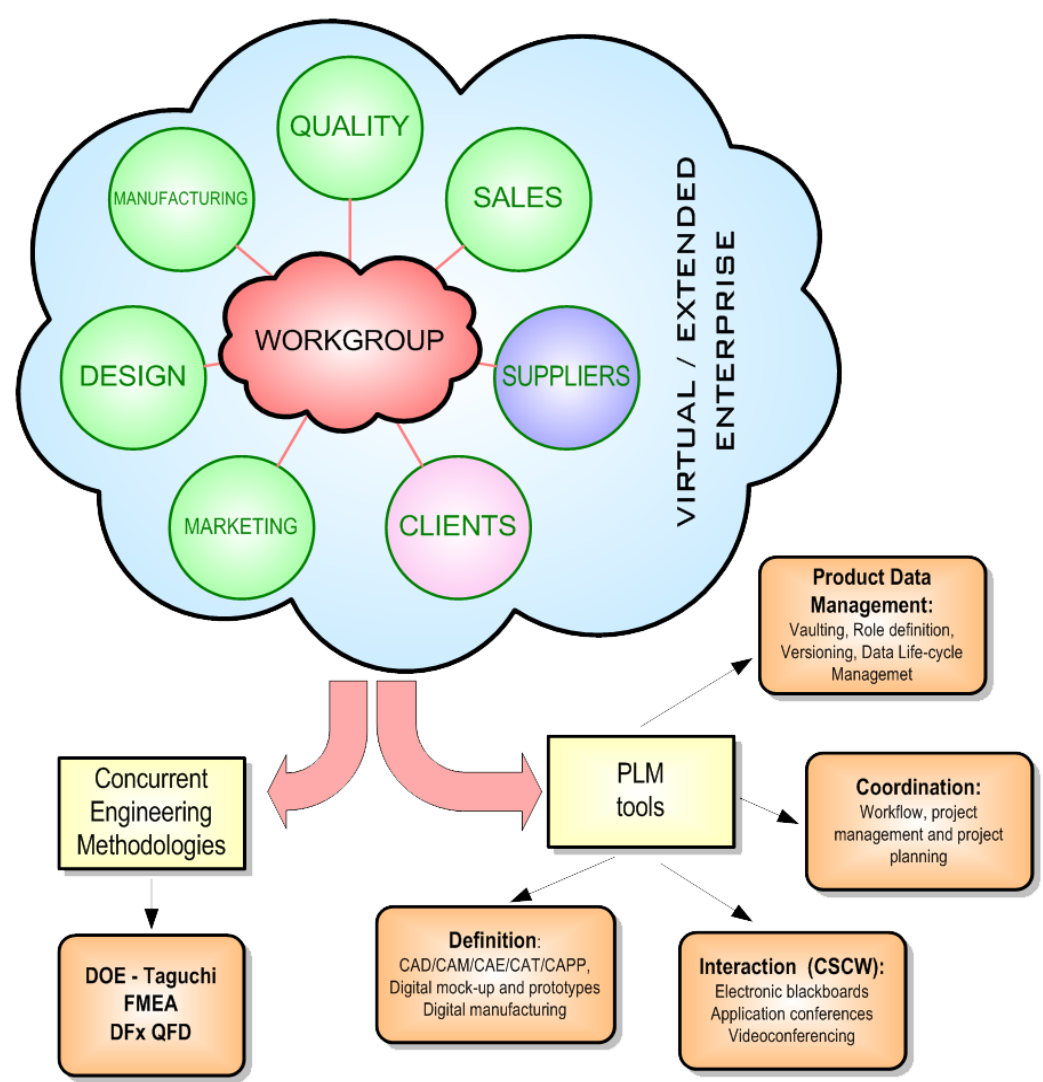

Figure 44. PLM in the context of the collaborative engineering paradigm

The PDM module is an essential building block of the PLM system. It serves as a central knowledge repository for process and product history, and promotes integration, collaboration, and data exchange among all stakeholders throughout the product life cycle. The PDM manages engineering data through better control of information, engineering activities, engineering changes, and product configurations.

Once product data is under control, users can streamline and automate product-related processes such as transmittals and engineering change orders. As PDM gets established, other departments and parts of the organization that play important roles in the product development cycle can also improve their performance. These improvements rely on a foundation of accurate, current engineering data that is made possible by the PDM system. 
There are three levels in a basic PDM structure, as shown in Figure 45. The first level is the information warehouse in which engineering information is stored. The information warehouse is responsible for functions such as data access, storage and recall, information security and integrity, concurrent use of data, and archival and recovery. It provides traceability of all actions taken on data.

The second level, the workgroup, provides user access, permissions, and control policies to the information stored in the information warehouse.

Finally, the third level provides an interface for users and programs to access the data. The level is responsible for user queries, user input, and report generation. It also provides interfaces for CAD programs to interact with the PDM.

It is in the context of PDM, specifically at the information warehouse level, where the proposed extended annotation model can be integrated. The goal is to provide a system to track and manage annotated models, so information from the annotations can be easily accessible.

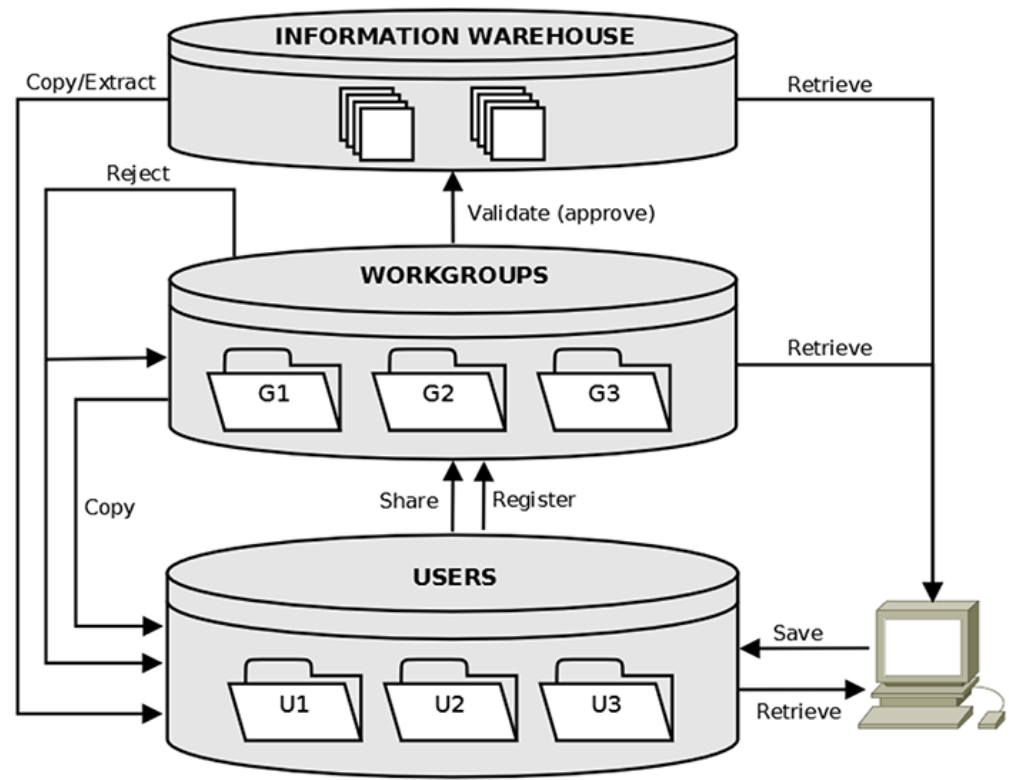

Figure 45. PDM structure 
Annotation Mechanisms to Manage Design Knowledge in Complex Parametric Models and their Effects on Alteration and Reusability

\section{PLM Integration}

In order to facilitate collaborative design and integration with existing systems, the database version of the extended annotation model was designed to be easily integrated as a module into a typical PLM system. The integration scheme is shown in Figure 46.

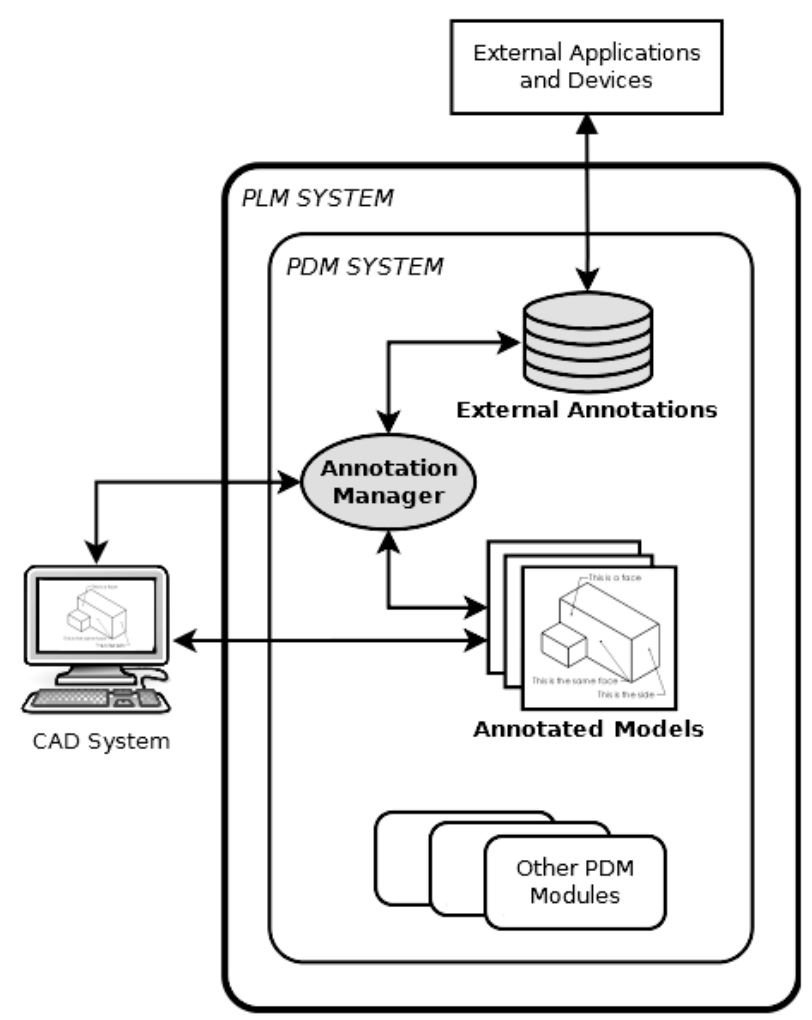

Figure 46. Integration architecture (arrows show information flow). Other PLM modules have been omitted for clarity

The interactive component of the annotation manager, which is devoted to the visualization, filtering, and manipulation of the model annotations, is still included as a local module or plug-in for the CAD system. This is the component that overloads the existing PMI functionalities in the CAD program to provide a more intuitive navigation for annotations. 
However, the external representation of the annotation is included into the Product Data Management (PDM) module of the PLM. The PDM system manages 3D CAD models (with the corresponding internal annotations, in this case) and the associated documentation. Therefore, it seems natural for the information from external annotations to be managed as an additional database in the PDM.

The synchronization component of the annotation manager is connected the Product Data Management (PDM) module of the PLM and accessible from the CAD application. The synchronization mechanisms are transparent to the user, as they are automatically launched in the background when an alteration is detected in the model.

In the case of users working locally, (i.e., disconnected from the annotations database) and connecting to the PLM system after some modifications have already been made to the model, the annotation manager compares the information from the model with the information from the PLM database and prompts the user on how to proceed. Changes can be synchronized from the model to the database, from the database to the model, or they can be combined (updating both the database and the model) by comparing the annotations and maintaining only the most recent version in both places.

The availability of the annotations in the PLM database provides a method to feed information to external applications, which can be beneficial in a variety of situations. For example, both content and quality of the annotations can be studied and evaluated as well as the interactions between users, with the purpose of determining what makes annotations effective in product design environments and how design knowledge can be represented. Additionally, annotations can be treated as "posts", similar to those used in social networking systems, and made available in a web-based system so designers can vote or comment on annotations based on their experiences. Knowledge derived from annotation information can ultimately be used to define sets of best modeling practices or as indicators of quality.

In addition, the integration of the extended annotation system with a PLM solution opens new opportunities for collaborative design, such as the video conferencing module described later in this chapter. 


\section{Annotation History}

To demonstrate the benefits of making annotation information externally available from the PLM database, an annotation history module (also integrated with the PLM system) was developed. This module keeps track of the changes and actions performed to the model annotations.

Due to the iterative and evolving nature of product design, models undergo constant change. Unfortunately, the information linked to a model is not always properly updated as the geometry changes, which often results in a poor or incomplete documentation of a particular design operation. Just as computer programmers comment the source code of a program to document, communicate, and recall specific changes and algorithms, engineers and product designers also need proper documentation to remember all the versions, design changes, and reasons for change, of specific components.

In this context, the annotation history module provides an automatic mechanism to save and manage information about particular design decisions, which can also motivate and incentivize designers to annotate their models. After all, historical annotation information may not just be valuable for future users of a model, but also for the original creators.

The annotation history module is comprised of a new table in the database (called "annotation history" table, as shown in Figure 47) that stores obsolete versions of the annotations, and a new event handler in the system that moves the old annotation (logging the specific action that was performed, e.g. the content was altered, the annotation was reattached to a new point, the annotation was deleted, etc.) to the history table every time a modification is detected and before the new annotation record is updated in the original database. Dates and user information of the person responsible for the change are also recorded, providing an audit trail that can be used to understand the evolution of a model, process historical data, analyze actions of the users involved in particular design decisions, and identify design problems.

Access to the annotation history is provided by the interactive plug-in of the CAD system, so users can monitor the changes performed to a specific annotation and have the information at hand (see Figures 48 and 49). 


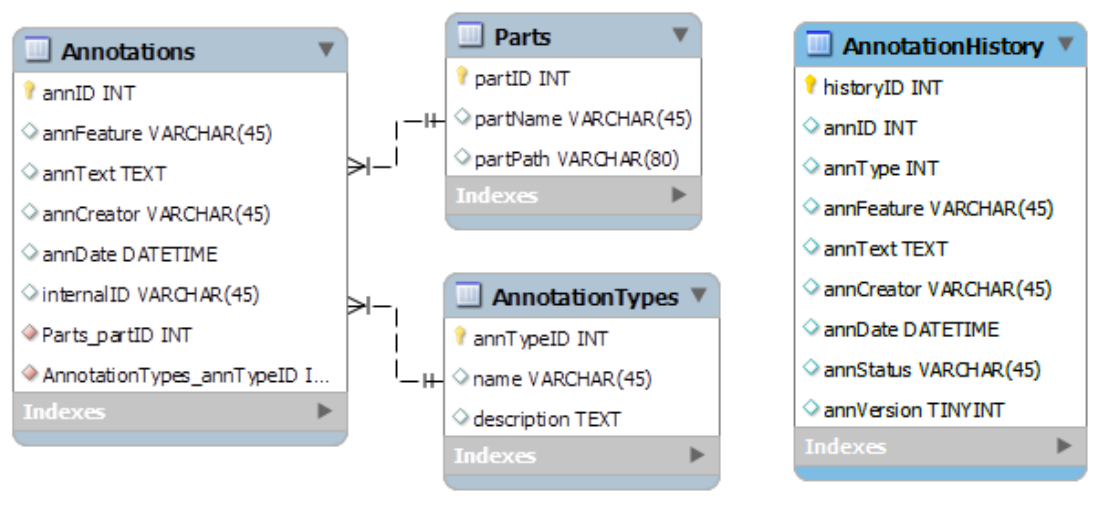

Figure 47. Enhanced Entity-Relationship (EER) database model for extended annotation system with history module

\begin{tabular}{|c|c|c|c|c|c|c|c|}
\hline Mode D & Database & \multicolumn{2}{|c|}{ DB Server localhost } & Use & & & \\
\hline Annotations & ions History & Search & Browser & & & & \\
\hline \multicolumn{8}{|c|}{$\square$ Filter } \\
\hline \multicolumn{8}{|c|}{24 Annotations found } \\
\hline Type & Feature & \multicolumn{3}{|c|}{ Annotation } & Creator & Date & - \\
\hline 1 & Boss-E... & \multicolumn{3}{|c|}{ Edit geometric constraint in sketchto modify overall angle } & Jorge & $6 / 16 / 2 \ldots$ & \\
\hline 1 & Boss-E... & \multicolumn{3}{|c|}{ Edit offset in Extrude to modifyspacing } & Jorge & $6 / 16 / 2 \ldots$ & \\
\hline 1 & Fillet2 & \multicolumn{3}{|c|}{ Modify fillet radius if body angle changes } & Jorge & $6 / 16 / 2 \ldots$ & \\
\hline 1 & Boss-E... & \multicolumn{3}{|c|}{ Ribs from two extrusionswith same sketch } & Jorge & $6 / 16 / 2 \ldots$ & \\
\hline 1 & Boss-E... & \multicolumn{3}{|c|}{ Ribs sketched on construction plane.Perpendicularity dep... } & Jorge & $6 / 16 / 2 \ldots$ & \\
\hline 1 & Cut-Ex... & \multicolumn{3}{|c|}{ Ensure correct dimensions of rib and filletsif modifying thi... } & Jorge & $6 / 16 / 2 \ldots$ & \\
\hline 1 & Boss-E... & \multicolumn{3}{|c|}{ Ribs on both sides may require update when side cuts ar... } & Jorge & $6 / 16 / 2 \ldots$ & \\
\hline 1 & $M 7 \times 1.0 \ldots$ & \multicolumn{3}{|c|}{ Hole distribution defined individually by size.Do not patter... } & Jorge & $6 / 16 / 2 \ldots$ & \\
\hline 1 & Boss-E... & \multicolumn{3}{|c|}{ Do not use symmetry. Angles may vary } & Jorge & $6 / 16 / 2 \ldots$ & $=$ \\
\hline 1 & Cut-Ex... & \multicolumn{3}{|c|}{ Ensure cut doesn't interfere with fillet } & Jorge & $6 / 16 / 2 \ldots$ & \\
\hline 1 & $M 4 \times 0.7 \ldots$ & \multicolumn{3}{|c|}{ Ensure standard metric sizes for all holes } & Jorge & $6 / 16 / 2 \ldots$ & \\
\hline 1 & Boss-E... & \multicolumn{3}{|c|}{ Ensure a minimum angle of 20 degrees } & Jorge & $6 / 16 / 2 \ldots$ & \\
\hline 1 & Not att... & \multicolumn{3}{|c|}{ Refer to Model AA0314for weight control information } & Jorge & $6 / 16 / 2 \ldots$ & \\
\hline 1 & Boss-E. & \multicolumn{3}{|c|}{ Modify angle to 25 degram } & Jorge & $6 / 16 / 2 \ldots$ & \\
\hline 1 & Fillet5 & \multicolumn{2}{|c|}{ Optional for weight con } & View History & Jorge & $6 / 16 / 2 \ldots$ & \\
\hline 1 & Not att... & \multicolumn{2}{|c|}{ Dimensional adjustment } & View Link & Jorge & $6 / 16 / 2 \ldots$ & \\
\hline 1 & Boss-E... & \multicolumn{2}{|c|}{ Stamp id number on this } & Add/Replace & Jorge & $6 / 16 / 2 \ldots$ & \\
\hline 1 & $M 4 \times 0.7 \ldots$ & \multicolumn{3}{|c|}{ Contact design center it...... } & Jorge & $6 / 16 / 2 \ldots$ & \\
\hline 1 & Not att... & \multicolumn{3}{|c|}{ Break all sharp edges to $\mathrm{R} 3$} & Jorge & $6 / 16 / 2 \ldots$ & \\
\hline 1 & Boss-E... & \multicolumn{3}{|c|}{ Modify extrude offset if overall depth changesto ensure $r . .}$. & Jorge & $6 / 16 / 2 \ldots$ & \\
\hline 1 & Boss-E... & \multicolumn{3}{|c|}{ Thickness increased afterinitial FEA test results } & Jorge & $6 / 16 / 2 \ldots$ & r \\
\hline 1 & & & III & 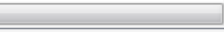 & & 1 & \\
\hline \multicolumn{8}{|c|}{ Sync Model to DB } \\
\hline Visualizatio & & & & & & & \\
\hline (-) Displa & lay All Annota & tions & & $\nabla$ Show Dimensions & & & \\
\hline Intera & active Visuali: & ization in 3 & 3D Model & $\square$ Show Only Assembly Ann & otations & & \\
\hline
\end{tabular}

Figure 48. Accessing history module 
Annotation Mechanisms to Manage Design Knowledge in Complex Parametric Models and their Effects on Alteration and Reusability

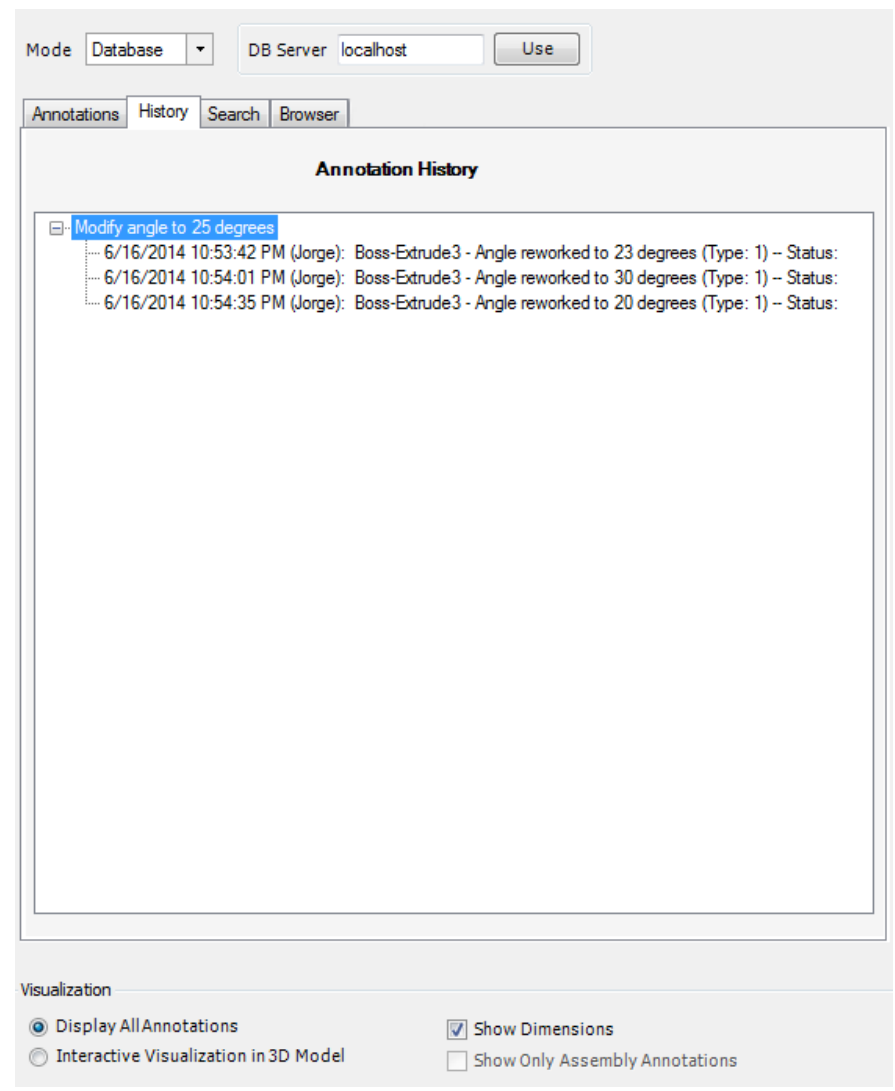

Figure 49. Annotation history records

Because historical annotation data is stored externally, information could also be made available via a comprehensive web-based interface connected to the PLM database. This would allow, for example, studying various aspects of design annotations from a communication and collaboration standpoint, such as assessing the evolution of communication networks in design teams, or analyzing specific types of team interactions overtime. In addition, the use of annotation history records also allows managers and supervisors to restore old annotations or revert back to previous annotation states of the model, if necessary. 


\section{Video Conferencing Component}

An additional component of the proposed system is a module that provides video conferencing functionality to the annotation manager. The module is designed as part of the extended annotation management system and introduces IP-based point-to-point video communication and screen sharing functionalities, a cost-effective solution that allows users to communicate with one another without relying on a central server.

This functionality is offered as an alternative to commercial video conferencing tools such as Skype ${ }^{\mathrm{TM}}$. The problems with using this type of commercial tools in corporate environments are numerous. Some of these problems include:

- Many video-conferencing tools are not standards-compliant, allowing any vulnerability to pass through corporate firewalls.

- Encryption are typically closed source and prone to man-in-themiddle attacks.

- Because the service is provided by an external source, outages, downtime, and security cannot be controlled by the organization.

- Numerous video conferencing tools have been reported to use excessive computer resources and network bandwidth.

- Many of the existing tools are untraceable and not auditable, putting organizations that are subject to compliance laws at risk.

For these and other reasons, some system administrators have banned video conferencing tools such as Skype ${ }^{\mathrm{TM}}$ on corporate, government, and education networks. The proposed video conferencing module provides a safe mechanism to contact other users by using a point-to-point system that gives corporations full control of their communications technology.

The main idea behind the video conferencing module is to provide a direct link between communication tools and model annotations, so user information is available "per annotation." In other words, users interacting with an annotated model can select an annotation, retrieve the contact information of the designer that created that specific annotation from the PLM system, and directly make a video call or share the screen with that particular user to ask questions and/or request additional information or explanations about the model. The entire workflow takes place within the interface of the CAD application. 
In a scenario where members of a design team are located in a corporate computer network and model the various components of a product using their CAD workstations, shared files and content are typically managed by a PLM system, which often acts as a version control system and file vault. In the context of the proposed communication module, the scope of the PLM software is extended to store the contact information of the participants that are involved in a particular task (such as designing a CAD assembly and/or individual parts). Participants may include model creators, users (of CAD models created by other participants), and annotators. Therefore, information regarding the participants of a specific project is linked to the CAD files of that project. This information includes the workstations' IP addresses and ports, which allows the communication module to identify users when establishing a call.

The architecture of the proposed communication system is shown in Figure 50. Dashed lines indicate regular traffic between the CAD application and the Product Data Management (PDM) component of the PLM system (to synchronize CAD files, upload and download updated files, etc). Solid lines represent the data flow generated to and from the annotation manager and the communications module.

When a user loads a CAD model in his/her workstation, the communications module automatically connects to the PLM system and requests the contact information of the team members that are associated to that specific model. The information, which is stored as a new table in a database managed by the PLM system, is retrieved as a list of names, IP addresses, and ports and displayed in the user's screen, directly within the CAD interface. Commands are available to establish video calls and shared screen sessions with the selected participants (see Figure 51). The combination of annotation and video communication tools becomes especially relevant in situations where heavily annotated models are used and multiple designers annotate the same model to communicate design intent and/or manufacturing information. In these cases, certain textual annotations may not be clear to some users, thus requiring further explanation or clarification by the annotators. Direct access from the model annotation to the user contact information is desirable. 


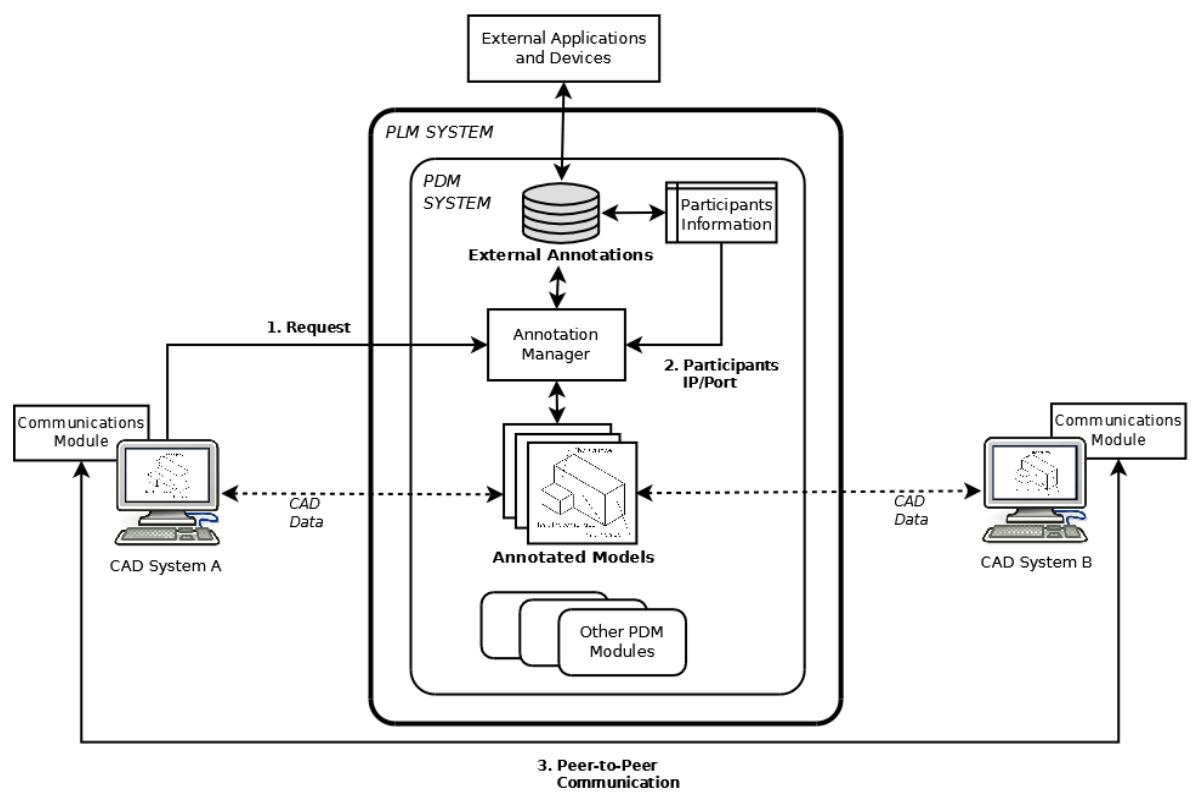

Figure 50. System architecture with video conferencing module

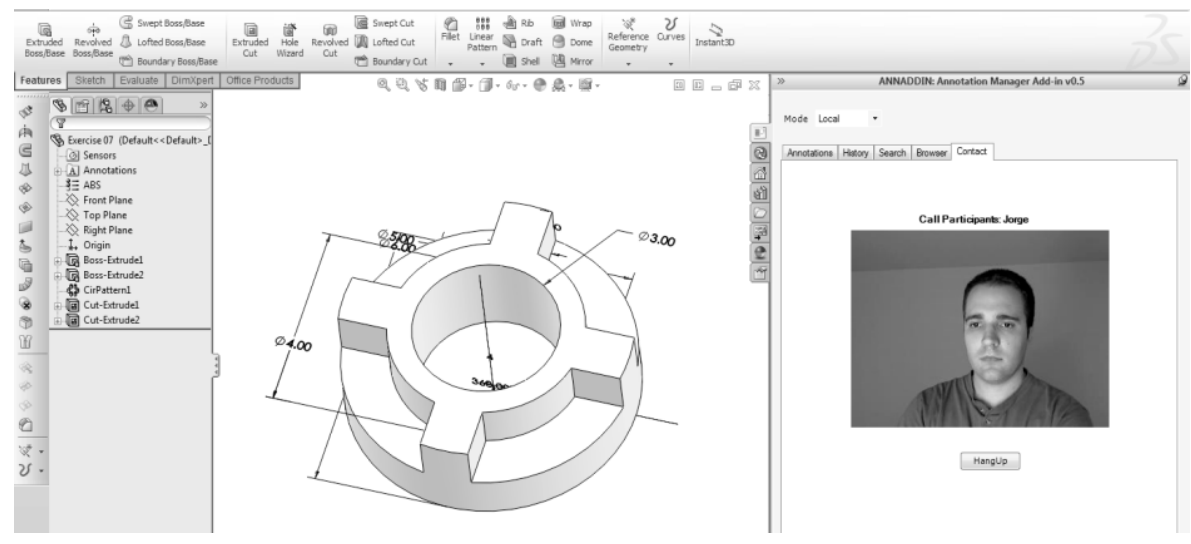

Figure 51. CAD interface (SolidWorks ${ }^{\circledR}$ ) with video conferencing module 
To remain responsive to remote requests, the module is implemented as a multi-threaded application. A "server" thread listens for incoming calls from other users, whereas a "client" thread initiates the communication. Because of the nature of the data being transmitted (real-time audio and video) a simple User Datagram Protocol (UDP) is used, which permits a continuous data stream without waiting for delay packets. Poor network conditions or reduced bandwidth can obviously impact the quality of the video, but the media stream will not stall due to packet loss or retransmission delays.

In the current implementation, both audio and video are transmitted uncompressed at a low resolution, which we consider acceptable for initial testing purposes. Future development plans include the incorporation of video compression techniques at high definition using the H.264 standard.

\subsection{Workflow}

The workflow of collaborative activities using the annotation manager with the video conferencing tool starts by navigating the visual clutter caused by the large amount of annotations in the model. Using the annotation manager, interaction with the annotated model is achieved via a graphical interface, in which the annotations associated to the model are displayed in a tabular form in the annotations area (including type, feature, creator, and date).

Selecting an item from the annotations area causes the corresponding annotation in the 3D model to automatically highlight, which provides an effective visual cue to the user. In addition, the annotation manager provides visual filtering and search functionalities.

Once the user identifies an annotation that requires contacting the annotator, a video conference session can be initiated by displaying the options linked to the annotation and selecting "Contact Annotator." The workflow is illustrated in Figure 52. 

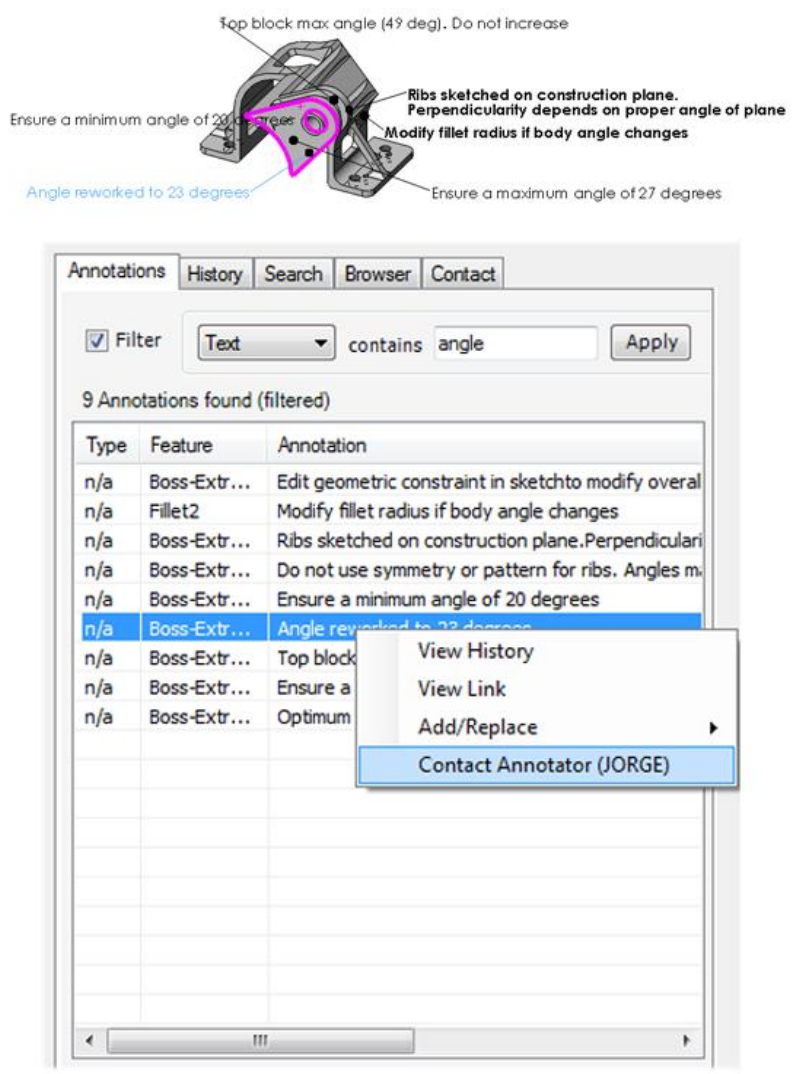

Figure 52. Contacting annotator

\section{Concluding Remarks}

In this chapter, we proposed an extension to the original extended annotation data structures and tools by integrating it with existing PLM systems. In addition, we have introduced a history module to keep track of annotation changes as the model evolves and an annotation-based video conferencing component for a more collaborative engineering where all user interactions, communication, and workflow occur through the existing CAD environment. All tools are integrated and available to the user from an already familiar software interface. 
Future development efforts will be dedicated to improve video quality and test different video compression systems, such as H.264. Also, performance testing and analysis of the impact in the modeling workflow is required for situations where multiple users are connected to the same conference session.

Additionally, we are interested in implementing video recording capabilities for subsequent processing and playback of the video stream, as well as incorporating automatic captions, so that the technical information discussed in the video call can be exported to textual formats and made available to other users and applications. The availability of this information in textual form can allow the use of automatic data mining and knowledge extraction applications. 


\section{Chapter VII \\ Conclusions and \\ Future Work}

\section{Summary of Achievements}

In this doctoral research, a set of parametric modeling methodologies and the use of model-annotation techniques as a method to carry design intent information have been examined. Formal parametric modeling strategies for reusability have been analyzed in terms of the CAD model's internal structure and adaptability to changes. In the context of the MBE paradigm, CAD models are not just a three dimensional geometric representation of a part, but the result of a specific modeling strategy that reflects and constrains the potential capabilities of the model for future modification and reuse.

It was concluded that a good methodology alone does not guarantee CAD efficiency and reusability. Once a part reaches a certain level of complexity, guidelines and modeling strategies are insufficient. In this regard, annotation-based strategies have proven to be a valuable method to communicate design information as long as certain mechanisms are properly established. For this research, the use of model annotations as a method to support design intent communication has been analyzed in scenarios where inadequate modeling assumptions can be made by designers and when a solution to a challenge must be selected and communicated when multiple options are available. User performance was assessed in situations where heavily annotated models are used. 
In all cases, results show statistically significant benefits of annotated models over non-annotated models. In addition, the challenges involved in implementing an annotation-based solution for design communication have been identified and discussed.

The main contribution of this doctoral work is an annotation-based model for design intent communication that supports users during the modeling process. The proposed solution is intended to provide a framework for designers to add and manage design information explicitly within a CAD model. The structure of the suggested model is presented as a natural extension to the annotation mechanisms defined by current model-based definition standards and implemented by modern CAD packages. Because of the limitations of the existing annotations structures available in PMI modules (visualization and interaction mechanisms such as filtering and searching are non-existent) a more flexible mechanism based on a dual representation is presented. A software prototype was implemented to test usability, acceptance, and the effects of annotated models in the product development process, particularly in the area of CAD model reusability.

The results reported in this dissertation contribute to increase productivity of CAD users in collaborative design environments by improving design intent communication through the use of an annotation-based mechanism in a product data quality context. The proposed solution is intended to add a dynamic feature to PLM systems to collect, represent, and manage new knowledge and ultimately impact productivity. The research objectives stated in Chapter 1 have been materialized in the following elements:

- Parametric model complexity was examined in terms of design methodology, modeling practices, reusability, and communication of design intent. A software tool that calculates the DSM representation of a parametric CAD model was developed as well as a series of complexity metrics.

- Two experimental studies were conducted to evaluate the role and effects of CAD model annotations as mechanisms to store, transfer, and communicate design intent information during the CAD modeling process, specifically on design reusability and alteration activities.

- The strengths and limitations of existing annotation tools available in modern $\mathrm{CAD}$ environments were evaluated and the structure of a 
new extended model was developed based on existing functionalities available in current PMI modules.

- The architecture of a software application that implements the extended annotation model was defined and tested in terms of user interaction and perception of design intent.

- Additional modules to improve communication in collaborative design environments were designed based on the proposed extended annotation technology.

\section{Contribution to Knowledge}

The creation of CAD models of complex parts requires a well-thought modeling strategy to ensure reusability and flexibility to adapt to design changes. However, high-level quality models are difficult to produce, partly because high-level best practices, like those aimed at emphasizing design intent, are rarely reinforced in every stage nor transmitted from stage to stage. In this context, some of the most relevant parametric modeling strategies developed over the years have been evaluated and compared in this study.

Nevertheless, once a model reaches a certain level of complexity in terms of number of features and interdependencies, guidelines and proper modeling strategies are insufficient, as they cannot guarantee proper communication of design intent. Therefore, it is necessary to implement other mechanisms to aid designers in creating more reusable models.

CAD models are a major source of design knowledge. This knowledge includes the CAD modeling process and the design intent implicit in these digital artifacts. However, managing this design knowledge in an efficient and simple way is a great challenge, but can be supported by knowledge management tools. These systems are important for obtaining a competitive advantage, but they are usually expensive and hard to use. Simpler mechanisms such as the extended annotation model proposed in this dissertation can be implemented, but it is important to define suitable structures to support them.

From a cost and flexibility standpoint, the most efficient alternative suggests overloading the PMI functionality to express both design intent and design rationale ideas through $3 \mathrm{D}$ text notes. This is a very attractive option for small and medium enterprises (SMEs) that are transitioning to the MBE 
Annotation Mechanisms to Manage Design Knowledge in Complex Parametric Models and their Effects on Alteration and Reusability

paradigm and lack the resources to develop custom applications or to implement integrated solutions, such as Questmap, Compendium [Shum et al., 2006], and DRed [Bracewell et al, 2009].

A detailed description of the contribution to knowledge of this research is reported next in the form of four points:

- Understanding parametric model complexity.

- Determining the effects of model annotations in design intent communication.

- Extended annotations model proposal and development of software prototype.

- Connection of extended annotation model to other communication tools in collaborative design environments.

\subsection{Understanding parametric model complexity}

Parametric CAD modeling is a mature and well established technology whose strength lies in its ability to define geometric features coupled with dimensional adjustability. The benefit of this combination is a much easier way to modify and reuse existing designs.

To create truly reusable models, expert CAD users use a combination of experience, insight, and education that allows them to structure models (and their design trees) in such a way that they minimize the brittleness problems caused by feature interdependencies. Brittleness problems cannot be completely eliminated, as models usually fall apart as soon as the user goes outside the scope of parametric changes that have been anticipated.

Understanding parametric model complexity and the structure of parametric CAD models is an important but challenging task and certainly a necessary step in the creation of reusable models. In this research, complexity was analyzed in terms of the internal structure of the model's design tree. Two representation and visualization methods (DSM and graph) and a set of metrics were used to compare formal modeling methodologies. The tools developed as a result of this work provide a practical mechanism to examine and study parametric models on the fly directly from the CAD interface.

\subsection{Determining the effects of annotations in design intent communication}

Forcing designers to adhere to modeling practices and strategies that have been found to be efficient (at least for a particular sector or enterprise) is an 
essential step towards creating quality and reusable models. However, with large complex models those guidelines are simply not enough, as high level design information is usually missing in communication from one stage to the next.

The role of 3D annotations as carriers of product information (Geometric Dimensioning and Tolerancing, material specifications, etc) within CAD models has been found successful and its use has increased partly because of the formalization of new standards and the popularization of the ModelBased Enterprise paradigm. The ability to communicate design intent information using annotations, however, has proven to be difficult, although there are reasons to believe they can serve this purpose effectively as long as proper mechanisms are in place [Alducin-Quintero et al., 2012].

This research takes a first step towards understanding and evaluating user's response to different CAD challenges when design annotations are present. Results show that users perform better when design intent information is explicitly available in the model as annotations. When users manipulate annotated models, even with no prior warning, they select more efficient modeling procedures and create models that are more reusable.

\subsection{Extended annotations model and development of software prototype}

In commercial CAD packages, practical implementation of model-based definition standards is available via PMI modules. These modules include tools with full support for simple 3D annotations. However, because of the nature of the data involved, representing design intent information in an efficient manner requires more robust annotation structures such as the one presented in this dissertation.

As opposed to existing approaches such as Compendium [Shum et al., 2006] or DRed [Bracewell et al, 2009], where independent systems are used to manage the annotations, the proposed annotation model represents a natural extension that builds on existing annotation mechanisms that are available in model-based definition standards and implemented by PMI modules in many commercial CAD systems. This model provides a framework to incorporate more semantic annotations to CAD models and manage the information externally.

Experiments conducted with a software implementation of the proposed annotation model show that users perform better in terms of interacting with the information when annotation visualization and management mechanisms are used. 
Annotation Mechanisms to Manage Design Knowledge in Complex Parametric Models and their Effects on Alteration and Reusability

\subsection{Connection of extended annotation model to other communication tools in collaborative design environments}

An objective of this research was to provide an alternative solution to conventional Knowledge Based Systems by incorporating the concept of extended annotation to existing CAD/PLM systems. In general, it is easier and less intimidating for designers and engineers to capture knowledge directly on the CAD model than it is by using text documents and traditional knowledge management systems.

Design annotation-based tools can overcome one of the biggest barriers in successful knowledge management: staff members' reluctance and motivation to do knowledge management. In addition, these tools are well adapted to the fact that knowledge transfer is in essence is a collaborative activity, where one person shares knowledge with others through one or more channels. Opening simple and effective new channels of knowledge transfer is precisely one of the goals of the collaborative software application solution proposal in the information and the extended annotation model presented in this dissertation. In addition, the flexibility of the proposed model allows the incorporation of other communication tools such as videoconferencing and its seamless integration with both the extended annotation model and the CAD environment.

\section{Summary of Findings}

In this section, an explicit correlation between the main objectives stated in Chapter 1 of this dissertation and the contribution to knowledge is stated.

Objective 1: Examine parametric model complexity in terms of design methodology, modeling practices, reusability, and communication of design intent.

This research has explored parametric model complexity by analyzing the internal structure of the model's design tree and by comparing formal modeling methodologies specifically designed for reusability. It has been shown that although an efficient modeling methodology is a necessary element to guarantee flexibility and reusability, other mechanisms must be implemented, as much of the design intent information cannot be communicated effectively due to the implicit nature of the model's design tree. 
Objective 2: Examine the role of CAD model annotations as a mechanism to store, transfer, and communicate design intent information.

In this research, the author has examined the role of CAD model annotations as carriers of design intent information by conducting an extensive literature review (Chapter II) and two experimental studies (described in Chapter IV). In all cases, results confirm the suitability of annotation structures to successfully perform this task.

Objective 3: Evaluate the strengths and limitations of existing annotation tools available in modern CAD environments.

The author has studied current model-based annotation standards such as such as ASME Y14.41-2003 and ISO 16792:2006, and commercial PMI modules that implement such standards. Overall, it has been shown that the existing annotation approach is valid to communicate design information, although certain limitations must be overcome. These limitations include storage, content, interface, visualization, and user motivation issues and are extensively discussed in Chapter V. These limitations comprise the focal points addressed by the extended annotations model described in section 3 of Chapter V.

Objective 4: Evaluate the overall efficiency and assess the effects of annotations on design reusability and model alteration activities.

In this research, the author has evaluated user's performance in simulated scenarios that involved the alteration of annotated CAD models. The objective was to find evidence of better responses and model quality when annotations were used to communicate design intent. The complete experimental studies are shown in Chapters IV and V. It has been shown that users perform better in terms of modeling quality, and reusability when design intent information is explicitly available as annotations.

Objective 5: Determine the relationship between annotated 3D CAD models and user perception in terms of understanding of design intent.

It has been shown that a more comprehensive type of model annotation, such as the one developed for this research and described in Chapter V, has significant effects on model understanding which directly impacts reusability and alteration tasks. Particularly, this research has exposed the limitations of current techniques for managing the visual clutter created by 3D annotations and confirmed the need for more intuitive methods for browsing and filtering. 
Annotation Mechanisms to Manage Design Knowledge in Complex Parametric Models and their Effects on Alteration and Reusability

Objective 6: Evaluate the role of model annotations on the communication of design intent information.

The results of the two experimental studies described in Chapter V and the ability to integrate other communication tools such as the ones described in Chapter VI confirm the value and effectiveness of the proposed annotation solution. The communications module, the annotation history module, and the PLM integration provide evidence of the value and functionality of an annotation-based information and communication system. The extended annotation model presented in this dissertation can be considered as a building block for a comprehensive annotation-based framework to manage design intent information directly from the CAD interface.

Objective 7: Define the architecture of a software system that implements annotation-based tools and manages design intent information in an explicit manner.

For this research, the author has introduced a broader and more interactive annotation structure and demonstrated the feasibility of this new model in terms of explicit representation and communication of design intent information. The architecture of a software system that can be fully integrated with existing PLM systems is also presented. A software prototype based on this architecture was implemented that enhances the functionalities of standard PMI modules in CAD packages, reduces visual clutter, and provides users with a more intuitive and efficient mechanism to interact with annotations. The validation studies conducted with this software prototype reveal the value of the annotations as carriers of design information when proper managing, filtering, and visualization mechanisms are in place.

\section{Conclusions}

This doctoral research has focused on some of the difficulties inherent in traditional model-based engineering systems and the current representation of design information. The author has addressed the concept of parametric model complexity and the potential of model annotations as communication elements to facilitate collaborative development, which can eventually lead to significant gains in terms of quality and impact on design model reuse and alteration. 
The author has proposed an innovative extension to the current annotation models and data structures that allows a more descriptive and complete representation of design information. The proposed model includes a dual representation (external and internal) of the annotation, methods for interactively manipulating design annotations, and an interface for connecting this information visually to the engineering model, transforming it into a more semantic element within the design process. The architecture of this system can be fully integrated with existing PLM solutions in collaborative environments. The proposed system can be a cost effective approach to knowledge reuse in industrial contexts, particularly for those companies transitioning to or implementing MBE solutions, and a dynamic feature to current PLM systems.

The conclusions of this research can be summarized as the following:

- Proper parametric modeling strategies are essential factors to create reusable models. However, as CAD models become more complex, good modeling strategies are not sufficient to guarantee flexibility and reusability. From an industrial standpoint, this is a fundamental issue as complex $\mathrm{CAD}$ models require significant efforts to be created. In many cases, these models cannot be reused due to poor design intent communication. Therefore, supporting mechanisms such as design annotations are necessary.

- The use of model annotations as design communication elements can improve CAD quality and impact collaborative development. Experimental results show that when users manipulate annotated models, even with no prior warning, they select more efficient modeling procedures and create models that are more reusable. Models annotated with design intent information provide a statistically significant value over non-annotated models.

- Existing annotation mechanisms, as defined by model based standards and implemented by PMI modules, are currently not appropriate to be used as carriers of design knowledge. Experimental studies show that users that interact with models with no annotation management mechanisms perform significantly worse than users who do have these mechanisms, which confirms the need for advanced methods to manage and interact with annotations.

- The proposed extended annotation model provides a method to incorporate design knowledge directly into CAD models. The model 
to function as an additional component of the MBE paradigm that can be integrated with existing PLM systems. The annotation model and the software architecture documented in this dissertation are respectively the theoretical and practical representations of the overall contribution.

\section{Limitations}

In this section, some of the limiting factors in this doctoral work are discussed. Limitations include experimental constraints and assumptions as well as technological limitations.

In terms of experimental constraints, the studies reported in this dissertation represent a first step towards understanding the full effects and relationships between annotation mechanisms and design intent communication. As an initial approach, the level of complexity of the CAD models used in the studies was low or average. Although there are reasons to believe that the benefits of annotation mechanisms increase as model complexity increases, there is a need for additional experiments with more complex models to try to estimate or quantify the effects.

An obvious factor that could affect the results of the experimental work presented in this document is the participants' level of CAD knowledge and expertise. For the experiments, a diverse group of undergraduate students were used. Although freshmen engineering students can be considered a low skilled population, they also comprise a homogeneous group. Senior students bring a higher skill level but with a more heterogeneous distribution. In this regard, a pending future task requires an experimental study with advanced CAD users in a real industrial setting. It should be noted that testing experimental tools such as the one proposed in a real industrial environment is difficult because of the daily workloads and routines of the personnel, inherent risks, and availability.

Additional limitations in the experimental studies can be attributed to the sample sizes. In an academic setting, it is difficult to gather a large group of participants with a similar knowledge of a specific parametric modeling tool. It is even more difficult in an industrial setting, where a company with a relatively large design department would be needed.

In terms of technological limitations, the proposed system is specifically designed to work with text as a common representation method for 
geometric design intent information that can be efficiently incorporated to a CAD model. Although how to represent design knowledge effectively is a fundamental issue in knowledge management, and sophisticated representation models and formats have been developed to support different design activities, the most relevant techniques and algorithms still rely on a textual representation of knowledge.

Nevertheless, the software system developed as part of this doctoral work provides support for additional types of information elements such as hyperlinks, graphical information, and links to external documents. A mechanism allows connecting these elements to the CAD model and presenting the information within the CAD environment. Further studies with non-textual type of design knowledge are certainly of great interest.

\section{Future Work}

This doctoral dissertation opens several research lines to continue the study of annotation-based mechanisms and their impact in design communication and CAD productivity. The results and annotation model presented in this document will serve as the starting point for project ANNOTA (Ref\#TIN2013-46036-C3-1-R), a proposal submitted to "Programa Estatal de Investigación, Desarrollo e Inovación Orientada a los Retos de la Sociedad (2013)" and recently approved for funding. The main objective of project ANNOTA is the evaluation of 3D annotations to improve semantic/pragmatic quality of CAD models. The main hypothesis is that enriched 3D annotated CAD models can be considered as knowledge carriers that support the conversion from tacit knowledge to explicit knowledge in topics such as design intent and design rationale communication.

\subsection{CAD Model Complexity}

In the area of CAD model complexity, we are interested in identifying specific geometric characteristics in designs that can aid users in selecting the most efficient strategy for creating CAD models. A more extensive and comprehensive analysis of these methodologies will be performed with a more diverse set of CAD models with different levels of complexity and number of features for each methodology with the intention of evaluating the effects of altering parent nodes and determine how easy it is to recover from rebuild errors. This data can provide valuable information to make informed decisions about modeling methodologies and best practices. 
As a related line of study, new effective methods to interact with complex interdependencies within CAD models are needed. A typical design tree usually provides a simplified view of the construction history of the model without detailed information about the underlying complexity of the parent/child relationships. Although a number of modern CAD systems provide tools for visualizing direct relations, more advanced tools are required to analyze model complexity in terms of the internal graph structure of the design tree (similar to dependency diagrams used in this dissertation). Since the early work of [Anderl \& Mendgen, 1998], only minor advances have been made in commercial CAD systems, where there is an evident lack of tools of this kind. Despite recent initiatives, additional work is required to analyze, measure, and manage CAD model complexity.

Finally, the author hopes this work will help lay the foundation to define parametric CAD complexity metrics, which could serve as simple indicators of model quality. These indicators could be employed to objectively evaluate CAD models, suggest better modeling alternatives, and automatically optimize models based on specific guidelines.

\subsection{Extended Annotation Processing}

The availability of information outside the model can be a valuable asset if we consider that a set of "best design practices" could be determined as a collaborative effort by using this information. For example, by incorporating social networking capabilities in the system, model annotations may be treated as "posts" that designers can "up-vote" or "down-vote" based on their experience and preferences. The result of these interactions can help rank and categorize product-related information in a variety of ways.

In addition, an external representation of extended annotations provides an information source for "software engineering" techniques, so the characteristics of the annotations such as language, writing style, and content can be analyzed. Research in the software engineering field has defined strategies for improving code reuse that can potentially inspire new methods for improving CAD model reuse and design intent communication. External representations could be treated in a manner that is similar to source code comments in software engineering. They could be processed and evaluated using quality metrics. Determining if users are providing too much information, too little, or simply not expressing the concept correctly can lead to the development of a standardized structure or language for CAD annotations related to design. 
The ultimate goal of the proposed extended model is to develop a framework to experiment with different annotation approaches with the objective of building a knowledge sharing and management system, where design intent and product-related information can be contained and integrated within an annotated solid model. In-context annotation information can significantly extend the role of CAD models in the design process, transforming them from mere carriers of geometric information to central players in most design documentation and knowledge sharing activities.

\subsection{User Interaction}

In terms of using interaction, we are interested in analyzing the effects of color and other perceptual elements such as annotation size, style, and patterns to define more sophisticated strategies that support visual searches.

Also, new techniques to simplify the creation and distribution of annotations among layers and groups are also desirable. Despite the clutter reduction observed in the study presented in chapter 5 when using annotation layers, the process of organizing information in groups has proven to be tedious and time consuming. Alternative studies with different groups based on alternative organization criteria could impact user performance and thus provide different outcomes. Ideally, a system that performed this task automatically or semi-automatically could potentially provide significant benefits to users of annotated CAD models.

\subsection{Further Experiments}

Although the results presented in this document suggest that users perform better in terms of modeling quality and reusability when design intent information is explicitly available as annotations, a more comprehensive study with a larger and wider variety of models, representing a more diverse range of design scenarios, and a larger and more experienced sample of CAD users is desired.

Similar studies can be conducted to evaluate whether annotations have a significant impact on design intent communication (so users perform better), even when the geometry modifications are exposed at the beginning of the exercise. In this study, participants would be shown the initial and final versions of a complex CAD model and asked to perform a longer sequence of alterations.

In addition, it will be interesting to compare user's performance when design intent is available as annotations versus when it is provided as a traditional 
document or included in an external repository. Further work is also required to evaluate user acceptance, motivation, and incentives regarding the annotation of models.

\subsection{Communication}

Although the communications module that provides videoconferencing functionalities to the proposed system was shown to a number of engineers and product designers in an informal setting (positive initial reactions were observed), a formal validation with real end-users and a comparative study with other communication tools are necessary.

As discussed in chapter 6, future efforts can be dedicated to improve streaming and video quality. Also, an analysis of the impact in the modeling workflow is required for situations where multiple users are connected to the same conference session.

Additionally, video recording capabilities and automatic captioning can be implemented for subsequent processing and playback of the video stream. This would allow users to export the information discussed in the video call to textual formats, so it can be managed and processed by other users and applications. The availability of the information in textual form can make this otherwise inaccessible information a data source for automatic data mining and knowledge extraction techniques. 



\section{REFERENCES}

Aberdeen Group (2006). The Transition from 2D Drafting to 3D Modeling Benchmark Report - Improving Engineering Efficiency. Aberdeen Group, Boston.

Abrahamson, S., Wallace, D., Senin, N., Sferro, P. (2000). Integrated design in a service marketplace. Computer-Aided Design, vol. 32(2), pp. 97-107.

Ahlberg, C., Shneiderman, B. (1994). Visual Information Seeking: Tight Coupling of Dynamic Query Filters with Starfield Displays. SIGCHI Conference on Human Factors in Computing Systems. pp. 313-317.

Ahmed, S., (2005). Encouraging reuse of design knowledge: A method to index knowledge. Design Studies, vol. 26(6), pp. 565-592.

Alducin-Quintero, G., Contero, M., Martín-Gutiérrez, J., Guerra-Zubiaga, D.A., Johnson, M.D. (2011). Productivity Improvement by Using Social-Annotations about Design Intent in CAD Modeling Process. Online Communities and Social Computing, pp.153-161

Alducin-Quintero, G., Rojo, A., Plata, F., Hernández, A., Contero, M. (2012). 3D Model Annotation as a Tool for Improving Design Intent Communication: A Case Study on Its Impact in the Engineering Change Process. In: Proceeding of the ASME International Design Engineering Technical Conferences and Computers and Information in Engineering Conference; pp. 349-356.

Ali, K., Hartmann, K., Strothotte, T. (2005). Label Layout for Interactive 3D Illustrations. 13th International Conference in Central Europe on Computer Graphics, Visualization and Computer Vision. Bory, Czech Republic

Ameri, F., Dutta, D. (2005). Product lifecycle management: closing the knowledge loops. Computer-Aided Design and Applications, vol. 2(5), pp. 577-590

Anderl, R., Mendgen, R., (1998). Analyzing and Optimizing Constraint-Structures in Complex Parametric CAD models. Geometric Constraint Solving and Applications, B. Brüderlin, \& D. Roller, eds. Springer-Verlar, New York, pp. 58-81.

ASME Y14.41-2012 Digital Product Definition Data Practices. The American Society of Mechanical Engineers, 2012, New York.

Au, C.K., Yuen, M.M.F., (2000). A Semantic Feature Language for Sculptured Object Modelling. Computer Aided Design, vol. 32(1), pp. 63-74.

Aubry, S., Thouvenin, I., Lenne, D., Olive, J., (2007). A knowledge model to read 3D annotations on a virtual mock-up for collaborative design. Proceedings of the 11th International Conference on Computer Supported Cooperative Work in Design.

Barclay, I., Dann, Z., (2000). New-Product-Development Performance Evaluation: A Product-Complexity-Based Methodology, IEE Science, Measurement and Technology, IEE Proceedings, vol. 147(3), pp. 41-55. 
Bashir, H.A., Thomson, V., (2001). An Analogy-Based Model for Estimating Design Effort. Design Studies, 22, pp. 157-167.

Bertoline, G.R., Wiebe, E.N., (2002). Fundamentals of Graphics Communication. (3rd. ed.) In Ridge B., Irwin R. D. (Eds.). McGraw-Hill.

Betting, B., Shah, J., (2005). Derivation of a Standard Set of Geometric Constrains for Parametric Modeling and Data Exchange. Computer-Aided Design, vol. 33(10), pp. 17-33.

Bilasco, I.M., Gensel, J., Villanova-Oliver, M., Martin, H., (2005). On indexing of 3D scenes using MPEG-7. Proceedings of the 13th annual ACM international conference on Multimedia. Hilton, Singapore, ACM.

Bilasco, I.M., Gensel, J., Villanova-Oliver, M., Martin, H., (2006). An MPEG-7 Framework Enhancing the Reuse of 3D Models. Proceedings of the eleventh international conference on 3D web technology. Columbia, Maryland, ACM.

Bly, S.A., Harrison, S.R., Irwin, S., (1993). Media Spaces: Bringing People Together in a Video, Audio and Computing Environment. Communications of the ACM, vol. 36(1), pp.28-46.

Bodein, Y., Rose, B., Caillaud, E., (2013). A Roadmap for Parametric CAD Efficiency in the Automotive Industry. Computer-Aided Design, vol 45(10), pp. 1198-1214.

Bodein, Y., Rose, B., Caillaud, E., (2014). Explicit Reference Modeling Methodology in Parametric CAD System. Computers in Industry 65(1), pp. 136147.

Boehm, B., Bayuk, J., Desmukh, A., Graybill, R., Lane, J.A., Levin, A., et al. (2010). Systems 2020 Strategic Initiative. DoD Systems Engineering Research Center, Technical Report SERC-2010-TR-009, August 29, 2010.

Boujut, J.F., Dugdale, J., (2006). Design of a 3D Annotation Tool for Supporting Evaluation Activities in Engineering Design. Cooperative Systems Design, COOP, vol 6, pp. 1-8.

Bracewell, R. H., Wallace, K. M., (2003). A Tool for Capturing Design Rationale. Proceedings of the 14th International Conference on Engineering Design (ICED'03). Design Society, Stockholm, Sweden.

Bracewell, R., Wallace, K., Moss, M., Knott, D., (2009). Capturing design rationale. Computer-Aided Design, vol. 41, pp. 173-186

Brissaud, D., Garro, O., Poveda, O., (2003). Design Process Rationale Capture and Support by Abstraction of Criteria. Research in Engineering Design, vol. 14, pp. 162-172. 
Annotation Mechanisms to Manage Design Knowledge in Complex Parametric Models and their Effects on Alteration and Reusability

Brush, A.J.B., Bargeron, D., Gupta, A., Cadiz, J.J., (2001). Robust annotation positioning in digital documents. Proceedings of the SIGCHI conference on Human factors in computing systems. Seattle, Washington, United States, ACM.

Burge, J.E., Brown, D.C. (2008). Software engineering using rationale. Journal of Systems and Software, vol. 81, pp. 395-413.

Buse, R.P.L., Weimer, W.R., (2010). Automatically documenting program changes. Proceedings of the IEEE/ACM international conference on Automated software Engineering, Antwerp, Belgium.

Chandrasegaran, S.K., Ramani, K., Sriram, R.D., Horváth, I., Bernard, A., Harik R.F., Gao, W., (2013). The evolution, challenges, and future of knowledge representation in product design systems. Computer-Aided Design, vol. 45(2), pp. 204-228.

Chandrasekaran, B., Goel, A., Iwasaki, Y., (1993). Functional representation as design rationale. Computer, vol. 26(1), pp. 48-56.

Chang, K.H., Silva, J., Bryant, I., (1999). Concurrent Design and Manufacturing for Mechanical Systems. Concurrent Engineering, vol. 7, pp. 290-308.

Chen, R.Q., Tang, M., Dong, J.X., Chou, S.C., (2005). Model compression and transmission in collaborative CAD. Proceedings of the Ninth International Conference on Computer Supported Cooperative Work in Design, IEEE, vol. 1, pp. 582-587.

Cheung, W.M., Schaefer, D., (2009). Product Lifecycle Management: State-of-theart and Future Perspectives"; Enterprise Information Systems for Business Integration in SME's: Technological, Organizational, and Social Dimensions, pp. $37-55$.

Chin, J.P., Diehl, V.A., Norman, K.L., (1988). Development of an Instrument Measuring User Satisfaction of the Human-Computer Interface. Proceedings of the SIGCHI Conference on Human factors in Computing Systems, pp. 213-218

Chudoba, K.M., Wynn, E., Lu, M., Watson-Manheim, M.B., (2005). How virtual are we? Measuring virtuality and understanding its impact in a global organization. Information Systems Journal, vol. 15, pp. 279-306.

Cipriano, G., Gleicher, M., (2008). Text Scaffolds for Effective Surface Labeling. IEEE Transactions on Visualization and Computer Graphics, vol. 14(6), pp. 16751682

Conklin, E.J., Yakemovic, K.C., (1991). A Process Oriented Approach to Design Rationale. Human-Computer Interaction, vol. 6, pp. 357-391.

Contero, M., Company, P., Vila, C., Aleixos, N., (2002). Product data quality and collaborative engineering. IEEE Computer Graphics and Applications, vol. 22(3), pp. 32-42. 
Davies, D., (2008). Representation of multiple engineering viewpoints in Computer Aided Design through computer-interpretable descriptive markup. Mechanical Engineering. University of Bath, Bath, UK, University of Bath.

Davies, D., McMahon C.A., (2006). Multiple Viewpoint Design Modelling through Semantic Markup. Proceedings of IDETC/CIE 2006, ASME 2006 International Design Engineering Technical Conferences and Computers and Information in Engineering Conference, Philadelphia, Pennsylvania, USA.

Ding, L., Davies, D., McMahon, C., (2008). Sharing Information Throughout a Product Lifecycle via Markup of Product Models. Proceedings of the ASME 2008 International Design Engineering Technical Conferences \& Computers and Information in Engineering Conference, IDETC/CIE 2008, Brooklyn, New York, USA.

Ding, L., Ball, A., Patel, M., Matthews, J., Mullineux, G., (2009). Strategies for the Collaborative Use of CAD Product Models. Proceedings of the 17th International Conference on Engineering Design, ICED09, vol. 8, pp. 123-134.

Ding, L., Davies, D., McMahon, C.A., (2009). The Integration of Lightweight Representation and Annotation for Collaborative Design Representation. Research in Engineering Design, vol. 20(3), pp. 185-200

Drury, J.L., Pfaff, M.S., Klein, G.L., Liu, Y., (2013). Decision Space Visualization: Lessons Learned and Design Principles. Human-Computer Interaction. Interaction Modalities and Techniques, pp. 658-667.

Du Plessis, M., (2008). What bars organisations from managing knowledge successfully? International Journal of Information Management, vol. 28, pp. 285292.

Durstewitz, M., Kiefner, B., Kueke, R., Putkonen, H., Repo, P., Tuikka, T., (2002). Virtual collaboration environment for aircraft design. Information Visualisation 2002, Proceedings. Sixth International Conference on Information Visualisation. IEEE, pp. 502-507.

Ellis, G., Bertini, E., Dix, A., (2005). The Sampling Lens: Making Sense of Saturated Visualisations. Extended Abstracts on Human Factors in Computing Systems. pp. 1351-1354, Portland, USA.

Ellis, G., Dix, A., (2006). Enabling Automatic Clutter Reduction in Parallel Coordinate Plots. IEEE Transactions on Visualization and Computer Graphics, vol. 12(5), pp. 717-723

Ellis, G., Dix, A., (2007). A Taxonomy of Clutter Reduction for Information Visualisation. IEEE Transactions on Visualization and Computer Graphics, vol. 13(6), pp. 1216-1223 
Annotation Mechanisms to Manage Design Knowledge in Complex Parametric Models and their Effects on Alteration and Reusability

Elshoff, J.L., Marcotty, M., (1982). Improving computer program readability to aid modification. Communications of the ACM, vol. 25, pp. 512-521.

FIATECH, 2010, Capital Projects Technology Roadmap, www.fiatech.org (last accessed 06-02-2014)

Fishkin, K., Stone, M.C., (1995). Enhanced Dynamic Queries via Movable Filters. SIGCHI Conference on Human Factors in Computing Systems, pp. 415-420

Fonseca, M.J., Henriques, E., Silva, N., Cardoso, T., Jorge, J.A., (2006). A collaborative CAD conference tool to support mobile engineering. Rapid Product Development (RPD’06), Marinha Grande, Portugal.

Frank, A.U., Timpf, S., (1994). Multiple Representations for Cartographic Objects in a Multi-scale Tree - An Intelligent Graphical Zoom. Computers \& Graphics, vol. 18(6), pp. 823-829

Frechette, S., Huang, P., (2010). Model Based Enterprise Technical Data Package Requirements, National Institute of Standards and Technology, NISTIR 7749.

Frechette, S.P. (2011). Model Based Enterprise for Manufacturing. Proceedings of the 44th CIRP International Conference on Manufacturing Systems.

Freeman, L., (1977). A Set of Measures of Centrality Based on Betweenness. Soc. Networks, vol. 40, pp. 35-41.

Frivold, T.J., Lang, R.E., Fong, M.W., (1995). Extending WWW for synchronous collaboration. Computer Networks and ISDN Systems, vol. 28(1), pp. 69-75

Fu, M.W., Ong, S.K., Lu, W.F., Lee, I.B.H., Nec, A.Y.C., (2003). An Approach to Identify Design and Manufacturing Features from a Data Exchanged Part Model. Computer Aided Design, vol. 35(11), pp. 979-993.

Fu, W.X., Bian, J., Xu, Y.M., (2013). A video conferencing system for collaborative engineering design. Applied Mechanics and Materials, vol. 344, pp. 246-252

Fuh, J.Y.H., Li, W.D., (2005). Advances in Collaborative CAD: The State-of-theArt. Computer Aided Design, vol. 37, pp. 571-581.

Gajewska, H., Kistler, J., Manasse, M.S., Redell, D., (1994). Argo: a system for distributed collaboration. Proceedings of the Second International Conference on Multimedia, San Francisco, CA, USA, pp. 433-440, ACM, New York, USA.

Ganeshan, R., Garrett, J., Finger, S., (1994). A Framework for Representing Design Intent. Design Studies, vol. 15(1), pp. 59-84.

Gebhard, R., (20130. A Resilient Modeling Strategy. Technical Presentation, Solid Edge University.

Gero, J., (1990). Design prototypes: a knowledge representation schema for design. AI Magazine, vol. 11(4), pp. 26-36 
Glass, R.L., (2003). Facts and Fallacies of Software Engineering. Addison-Wesley.

Götzelmann, T., Hartmann, K., Strothotte, T., (2006). Agent-Based Annotation of Interactive 3D Visualizations. Smart Graphics. LNCS, vol. 4073, pp. 24-35. Springer Berlin Heidelberg

Gowan, Jr J.A., Downs, J.M., (1994). Video conferencing human-machine interface: a field study. Information and Management, vol. 27(6), pp. 341-356

Gravino, C., Risi, M., Scanniello, G., Tortora, G., (2011). Does the Documentation of Design Pattern Instances Impact on Source Code Comprehension? Results from Two Controlled Experiments. Proceedings of the 18th Working Conference on Reverse Engineering.

Greenberg, S., (1991). Computer-supported cooperative work and groupware: an introduction to the special issue. International Journal of Man-Machine Studies, vol. 34(2), pp. 133-141

Guerra D., Young R., (2006). A Manufacturing Model to Enable Knowledge Maintenance in Decision Support Systems, Journal of Manufacturing Systems, vol. 25 (2), pp. 122-136

Guerra D., Young R., (2008). Information and Knowledge Interrelationships in a Manufacturing Model, International Journal of Advanced Manufacturing Technology, vol. 39 (1), pp. 182-198. DOI 10.1007/s00170-007-1194-6

Guerra-Zubiaga D.A., Young R.I.M., (2008). Design of a Manufacturing Knowledge Model, International Journal of Computer Integrated Manufacturing. Vol. 12 (5), pp. 526-539. DOI: 10.1080/09511920701258040

Gunn, T.G., (1982). The mechanization of design and manufacturing. Scientific American, vol. 247(3), pp. 86-108

Haouari, D., Sahraoui, H., Langlais, P., (2011). How Good is Your Comment? A Study of Comments in Java Programs. Internation Symposium on Empirical Software Engineering and Measurement (ESEM), pp. 137-146

Hanratty, PJ. (1995). Parametric/relational solid modelling. In D. E. LaCourse (Ed.), Handbook of solid modeling, pp. 8.1-8.25. New York: McGraw-Hill.

Harding, J.A., (1996). A knowledge representation model to support concurrent engineering team working. Loughborough, Loughborough University of Technology

Harding, J.A., Popplewell, K., Fung, R.Y.K., Omar, R.A., (2001). An intelligent information framework relating customer requirements and product characteristics, Computers in Industry, vol. 44, pp. 51-65

Hartman, N.W., (2005). Defining Expertise in the Use of Constraint-Based CAD Tools by Examining Practicing Professionals. Engineering Design Graphics Journal, vol. 69(1), pp. 6-15 
Annotation Mechanisms to Manage Design Knowledge in Complex Parametric Models and their Effects on Alteration and Reusability

Henderson, M.R., (1993). Representing Functionality and Design Intent in Product Models. Proceedings on the second ACM Symposium on Solid modeling and Applications, pp. 387-396

Herron, J.B., (2013). Re-Use Your CAD: The Model-Based CAD Handbook. Action Engineering.

Horvath, L., Rudas, I.J., (2003). Modeling Behavior of Engineering Objects Using Design Intent Model. In: Industrial Electronics Society, IECON'03. 29th Annual Conference of the IEEE, vol. 1, pp. 872-876

INCOSE. International Council on Systems Engineering 2007, Systems Engineering Vision 2020, INCOSE-TP-2004-004-02.

ISO 16792:2006 Technical Product Documentation - Digital Product Definition Data Practices. Organisation Internationale de Normalisation, 2006, Genève, Suisse.

Iyer, N., Jayanti, S., Lou, K., Kalyanaraman, Y., Ramani, K., (2005). Shape-based Searching for Product Lifecycle Applications. Computer-Aided Design, vol. 37(13), pp. 1435-1446.

Iyer, G.R., Mills, J.J., (2006). Design Intent in 2D CAD: Definition and Survey. Computer-Aided Design and Applications, vol. 3(1-4), pp. 259-267

Jackson, C., Buxton, M., (2007). The Design Reuse Benchmark Report: Seizing the Opportunity to Shorten Product Development. Aberdeen Group, Boston.

Johnson, M.D., Diwakaran, R.P., (2011). An Educational Exercise Examining the Role of Model Attributes on the Creation and Alteration of CAD Models. Computers \& Educational, vol. 57(2), pp. 1749-1761.

Jung, T., Gross, M.D., Do, E.Y.L., (2002). Sketching annotations in a 3D web environment. In $\mathrm{CHI} 02$ Extended Abstracts on Human Factors in Computing Systems, pp. 618-619

Kajko-Mattsson, M., (2001). The State of Documentation Practice within Corrective Maintenance. Proceedings of the IEEE International Conference on Software Maintenance (ICSM'01).

Kao, Y.C., Lin, G.C.I., (1998). Development of a collaborative CAD/CAM system. Robotics and Computer-Integrated Manufacturing, vol. 14(1), pp.55-68

Karsenty, L., (1996). An Empirical Evaluation of Design Rationale Documents. Proceedings of the SIGCHI Conference on Human Factors in Computing Systems, pp. 150-156.

Kellens, A., Noguera, C., Schutter, K.D., Roover, C.D., D'Hondt, T., (2010). Coevolving Annotations and Source Code through Smart Annotations. Proceedings of the 2010 14th European Conference on Software Maintenance and Reengineering 
Khamis, N., Witte, R., Rilling, J., (2010). Automatic quality assessment of source code comments: the JavadocMiner. Proceedings of the Natural language processing and information systems, and 15th international conference on Applications of natural language to information systems, Cardiff, UK

Kiryakov, A., Popov, B., Terziev, I., Manov, D., Ognyanoff, D., (2004). Semantic annotation, indexing, and retrieval. Web Semantics: Science, Services and Agents on the World Wide Web, vol. 2(1), pp. 49-79.

Kitamura, Y., Washio, N., Koji, Y., Mizoguchi, R., (2006). Towards Ontologies of Functionality and Semantic Annotation for Technical Knowledge Management. New Frontiers in Artificial Intelligence: Joint JSAI 2005 Workshop PostProceedings, Berlin / Heidelberg.

Konduri, G., Chandrakasan, A., (1999). A framework for collaborative and distributed web-based design. Proceedings of the 36th annual ACM/IEEE Design Automation Conference. ACM, pp. 898-903

Koschutzki, D., Lehmann, K.A., Peeters, L., Richer, S., Tenfelde-Podehl, D., Zlotowski, O., (2005). Centrality Indices. Network Analysis: Methodological Foundations, U. Brandes, and T. Erlebach, eds., Springer Verlag, New York, NY.

Kunz, W., Rittel, H.W.J., (1970). Issues as elements of information systems. Center for Planning and Development Research. University of California at Berkeley

Kydd, C.T., Ferry, D.L., (1994). Managerial use of video conferencing. Information and management, vol. 27(6), pp. 369-375

Landers, D.M., Khurana, P., (2004). Horizontally-Structured CAD/CAM Modeling for Virtual Concurrent Product and Process Design. US Patent 6,775,581.

Lee, C.C., Yang, J., (2000). Knowledge Value Chain. Journal of Management Development, vol. 19(9), pp. 783-794

Leizerowicz, W., Lin, J., Fox, M.S., (1996). Collaborative design using WWW. Proceedings of the WET-ICE'96, CERC, University of West Virginia

Lenne, D., Thouvenin, I., Aubry, S., (2009). Supporting Design with 3DAnnotations in a Collaborative Virtual Environment. Research in Engineering Design, vol. 20(3), pp. 149-155

Li, W.D., Lu, W.F., Fuh, J.Y., Wong, Y.S., (2005). Collaborative computer-aided design-research and development status. Computer-Aided Design, vol. 37(9), pp. 931-940

Li, C., McMahon, C., Newnes, L., (2009). Annotation in Product Lifecycle Management: A Review of Approaches. Proceedings of the ASME International Design Engineering Technical Conferences and Computers and Information in Engineering Conference, DETC2009. Vol. 2. New York: ASME, pp. 797-806. 
Annotation Mechanisms to Manage Design Knowledge in Complex Parametric Models and their Effects on Alteration and Reusability

Liang, Y., Liu, Y., Kwong, C.K., Lee, W.B., (2012). Learning the "Whys": Discovering design rationale using text mining-An algorithm perspective. Computer-Aided Design, vol. 44(10), pp. 916-930

Liker, J.K., Fleischer, M., Arnsdorf, D., (1992). Fulfilling the Promises of CAD. Sloan Management Review, vol. 33(3), pp. 74-86

Londono, F., Cleetus, K.J., Nichols, D.M., Iyer, S., Karandikar, H.M., Reddy, S.M., Potnis, S.M., Massey, B., Reddy, A., Ganti, V., (1992). Coordinating a virtual team. CERC-TR-RN-92-005, Concurrent Engineering Research Centre, West Virginia University, West Virginia.

Lubell, J., Hartman, N., Cheney, D., (2011). Model Based Engineering Standardization and Validation. NIST Interagency/Internal Report (NISTIR) - 7796

Lubell, J., Chen, K., Horst, J., Frechette, S., Huang, P., (2012). Model Based Enterprise/Technical Data Package Summit Report. NIST Technical Note 1753

Mandorli, F., Otto, H.E., (2013). Negative Knowledge and a Novel Approach to Support MCAD Education. Computer-Aided Design and Applications, vol. 10(6), pp. $1007-1020$

Mathieson, J.L., Summers, J.D., (2010). Complexity Metrics for Directional NodeLink System Representations: Theory and Applications. Proceedings of the ASME International Design Engineering Technical Conferences \& Computers and Information in Engineering Conference, IDETC/CIE 2010.

Matthews, J., Ding, L., Feldman, J., Mullineux, G., (2009). The Maintenance and Handling of Constraints in Machine Design. Proceedings of the ASME 2009 International Design Engineering Technical Conferences \& Computers and Information in Engineering Conference, IDETC/CIE 2009, San Diego, California, USA.

Maxfield, J., Fernando, F.T., Dew, P., (1998). A distributed virtual environment for collaborative engineering. Presence: Teleoperators and Virtual Environments, vol. 7(3), pp. 241-261

May, A., Carter, C., (2001). A case study of virtual team working in the European automotive industry. International Journal of Industrial Ergonomics, vol. 27(3), pp. 171-186

MBE, 2014. Official Group for Model-Based Enterprise. http://model-basedenterprise.org (July 7, 2014).

MIL-STD-31000A, US Department of Defense Standard Practice: Technical Data Packages (2013)

Moran, T.P., Carroll, J.M., (1996). Design rationale: concepts, techniques, and use. Mahwah (New Jersey): Lawrence Erlbaum Associates, Inc. 
Myers, K., Zumel, N., Garcia, P., (2000). Acquiring Design Rationale Automatically. Artificial Intelligence for Engineering Design, Analysis and Manufacturing, vol. 14(2), pp. 115-135.

Nielsen, J., Mack, R.L., (1994). Usability inspection methods. Wiley.

Norman, D.A., (2002). The design of everyday things. Reprint ed. Basic Books

Noyes, L. (1980). The Positioning of Type on Maps: The Effect of Surrounding Material on Word Recognition Time. Human Factors, vol. 22(3), pp. 353-360

Olson, J.S., Olson, G.M., Meader, D.K., (1995). What mix of video and audio is useful for small groups doing remote real-time design work? Proceedings of the SIGCHI conference on Human factors in computing systems. ACM Press/AddisonWesley Publishing Co.

Ovsiannikov, I.A., Arbib, M.A., McNeill, T.H., (1999). Annotation technology. International Journal of Human-Computer Studies.

Palmer, J., (1994). Set-size Effects in Visual Search: the Effect of Attention is Independent of the Stimulus for Simple Tasks. Vision Research, vol. 34, pp. 1703 1721

Patel, M., Ball, A., Ding, L., (2008). Curation and Preservation of CAD Engineering Models in Product Lifecycle Management. Conference on Virtual Systems and Multimedia Dedicated to Digital Heritage (VSMM'08), University of Bath, pp. 5966

Patel, M., Ball, A., Ding, L., (2009). Strategies for the Curation of CAD Engineering Models. International Journal of Digital Curation, vol. 4(1), pp. 84-97.

Pena-Mora, F., Sriram, D., Logcher, R., (1993). SHARED-DRIMS: SHARED Design Recommendation-Intent Management System. Enabling Technologies: Infrastructure for Collaborative Enterprises, Proceedings, Second Workshop, pp. 213-221.

Pfleeger, S.L., Atlee, J.M., (2009). Software Engineering: Theory and Practice: Prentice Hall

Pittarello, F., De Faveri, A., (2006). Semantic description of 3D environments: a proposal based on web standards. Proceedings of the 11th international conference on 3D web technology, pp. 85-95

Prechelt, L., Unger-Lamprecht, B., Philippsen, M., Tichy, W.F., (2002). Two Controlled Experiments Assessing the Usefulness of Design Pattern Documentation in Program Maintenance. IEEE Transactions on Software Engineering, vol. 28, pp. 595-606. 
Annotation Mechanisms to Manage Design Knowledge in Complex Parametric Models and their Effects on Alteration and Reusability

Qiang, L., Zhang, Y.F., Nee, A.Y.C., (2001). A distributive and collaborative concurrent product design system through the WWW/Internet. The International Journal of Advanced Manufacturing Technology, vol. 17(5), pp. 315-322

Quereshi, S., Shah, J., Urban, S.D., Harter, E., Parazzoli, C., et al., (1997). Integration model to support archival of design history in databases. Proceedings of the 1997 ASME design engineering technical conferences and computers in engineering conference.

Quintana, V., Rivest, L., Pellerin, R., Venne, F., Kheddouci, F., (2010). Will modelbased definition replace engineering drawings throughout the product lifecycle? A global perspective from aerospace industry. Computers in Industry, vol. 61(5), pp. 497-508

Quintana, V., Rivest, L., Pellerin, R., (2012). Measuring and improving the process of engineering change orders in a model-based definition context. International Journal of Product Lifecycle Management, vol. 6(2), pp. 138-160

Rahman, S.M., Sarker, R., Bignall, B., (1999). Application of multimedia technology in manufacturing: a review. The Journal of Computers in Industry, vol. 38(1), pp. 43-52

Regli, W.C., Hu, X., Atwood, M., Sun, W., (2000). A Survey of Design Rationale Systems: Approaches, Representation, Capture and Retrieval. Engineering with Computers, vol. 16, pp. 209-235

Robertson, D., Allen, T.J., (1993). CAD-System Use and Engineering Performance. IEEE Transactions on Engineering Management, vol. 40, pp. 274-282.

Rodriguez, J., Ridge, J., Dickinson, A., Whitwam, R., (1998). CAD Training Using Interactive Computer Sessions. Proceedings of the ASEE Annual Conference and Exposition

Rosenholtz, R., (2001). Search asymmetries? What search asymmetries? Perception \& Psychophysics, vol. 63(3), pp. 476-489

Rosenholtz, R., Li, Y., Mansfield, J., Jin, Z., (2005). Feature Congestion: A Measure of Display Clutter. SIGCHI Conference on Human Factors in Computing Systems, pp. $761-770$

Salehi, V., McMahon, C., (2009). Action Research into the Use of Parametric Associative CAD Systems in an Industrial Context. Proceedings of the 17th International Conference on Engineering Design (ICED'09), vol. 5, M. Norell Bergendahl, et al., eds. pp. 133-144

Sandberg, S., Näsström, M., (2007). A Proposed Method to Preserve Knowledge and Information by Use of Knowledge Enabled Engineering. Proceeding of the ASME International Design Engineering Technical Conferences \& Computers and Information in Engineering Conference, Las Vegas, Nevada, USA. 
SASIG 2005 (Strategic Automotive Product Data Standards Industry Group) Product Data Quality Guidelines for the Global Automotive Industry recommendation version 2.1 [internet]. [cited 2013 Aug 2]. Available from: http://www.odette.se/

SASIG 2008, Strategic Automotive Product Data Standards Industry Group, 3D Annotated Model Standard, in JAMAEIC0422008: Japan

Scanniello, G., Gravino, C., Risi, M., Tortora, G., (2010). A controlled experiment for assessing the contribution of design pattern documentation on software maintenance. Proceedings of the 2010 ACM-IEEE International Symposium on Empirical Software Engineering and Measurement, Bolzano-Bozen, Italy

Schreck, D., Dallmeier, V., Zimmermann, T., (2007). How Documentation Evolves Over Time. 9th International Workshop on Principles of Software Evolution (IWPSE 2007), Dubrovnik, Croatia.

Shah, J.J., Runger, G., (2011). Misuse of Information-Theoretic Dispersion Measures as Design Complexity Metrics. ASME International Design Engineering Technical Conferences and Computers and Information in Engineering Conference, ASME, Washington, DC, DETC2011/DTM-48295

Shen, W., Hao, Q., Li, W., (2008). Computer Supported Collaborative Design: Retrospective and Perspective. Computers in Industry, vol. 59, pp. 855-862

Shneiderman, B., Plaisant, C., (2009). Designing the User Interface: Strategies for Effective Human-Computer Interaction. 5th ed. Addison Wesley

Shum, S.J.B., Selvin, A.M., Sierhuis, M., Conklin, J., Haley, C.B., Nuseibeh, B., (2006). Hypermedia Support for Argumentation-Based Rationale: 15 Years on from Gibis and Qoc. Rationale Management in Software Engineering, pp. 111-132

Song, H., Guimbretière, F., Hu, C., Lipson, H., (2006). ModelCraft: capturing freehand annotations and edits on physical 3D models. Proceedings of the 19th annual ACM symposium on User interface software and technology, pp. 13-22

Souza, S.C.B., Anquetil, N., Oliveira, K.M., (2006). Which documentation for software maintenance? Journal of the Brazilian Computer Society, vol. 12, pp. 31-44

Stein, T., Décoret, X., (2008). Dynamic Label Placement for Improved Interactive Exploration. 6th International Symposium on Non-Photorealistic Animation and Rendering, pp. 15-21

Steward, D.V., (1981). The design structure system: A method for managing the design of complex systems. IEEE Trans Eng Management, vol. 28(3), pp. 71-74

Storey, M.A., Ryall, J., Singer, J., Myers, D., Cheng, L.T., Muller, M., (2009). How Software Developers Use Tagging to Support Reminding and Refinding," IEEE Transactions on Software Engineering, vol. 35, pp. 470-483 
Annotation Mechanisms to Manage Design Knowledge in Complex Parametric Models and their Effects on Alteration and Reusability

Summers, J.D., Ameri, F., (2008). An Algorithm for Assessing Design Complexity Through a Connectivity View. Proceedings of the Tools and Methods for Competitive Engineering (TMCE)

Sung, R., Ritchie, J.M., Rea, H.J., Corney, J., (2011). Automated Design Knowledge Capture and Representation in Single-User CAD Environments. Journal of Engineering Design, vol. 22(7), pp. 487-503

Szykman, S., Sriram, R.D., Bochenek, C., Racz, J.W., Senfaute, J., (2000). Design Repositories: Engineering Design's New Knowledge Base. IEEE Intelligent Systems, vol. 15(3), pp. 48-55

Szykman, S., Sriram, R., Regli, W., (2001). The Role of Knowledge in NextGeneration Product Development Systems. Journal of Computing and Information Science in Engineering, vol. 1(1), pp. 3-11

Tan, L., Yuan, D., Krishna, G., Zhou, Y., (2007). /*icomment: bugs or bad comments?*. SIGOPS Operating Systems Review, vol. 41, pp. 145-158

Tay, F.E.H., Ming, C., (2001). A shared multi-media design environment for concurrent engineering over the internet. Concurrent Engineering, vol. 9(1), pp. 5563

Tay, F.E.H., Roy, A., (2003). CyberCAD: a collaborative approach in 3D-CAD technology in a multimedia-supported environment. Computers in Industry, vol. 52(2), pp. 127-145

Thouvenin, I., Guenand, A., Lenne, D., Aubry, S., (2005). Knowledge integration in early design stages for collaboration on a virtual mock up, Piscataway, NJ 08855 1331, United States, IEEE.

Tornincasa, S., Di Monaco, F., (2010). The future and the evolution of CAD. 14th international research/expert conference: trends in the development of machinery and associated technology, Mediterranean Cruise

Toussaint, J., Cheng, K., (2002). Design agility and manufacturing responsiveness on the web. Integrated Manufacturing Systems, vol. 13(5), pp. 328-339

Tufte, E.R., (1983). The Visual Display of Quantitative Information.Graphics Press, Cheshire, CT.

Ullman, D.G., (2010). The Mechanical Design Process, 4th ed. McGraw Hill, Boston

Umeda, Y., Ishii, M., Yoshioka, M., Tomiyama, T., (1996). Supporting conceptual design based on the function-behavior-state modeler. Artificial Intelligence for Engineering Design, Analysis and Manufacturing, vol. 10(4), pp. 275-288

Upton, D.M., Mcafee, A., (1999). The real virtual factory. Harvard Business School Press, pp. 69-89 
Ushakov, D., (2008). Variational Direct Modeling: How to keep design intent in history-free CAD. White Paper, LEDAS Ltd (2008).

Van De Vanter, M.L., (2002). The documentary structure of source code. Information and Software Technology, vol. 44, pp. 767-782

VDA Recommendation 4955/4.1: Scope and Quality of CAD/CAM Data. German Association of the Automotive Industry (VDA), Frankfurt, Germany, 2006.

Vila, C., Estruch, A., Siller, H.R., Abellán, J.V., Romero, F., (2007). Workflow methodology for collaborative design and manufacturing. Cooperative Design, Visualization, and Engineering, pp. 42-49, Springer Berlin Heidelberg

Wang, S., (2005). Annotation persistence over dynamic documents. Department of Civil and Enviromental Engineering, MIT. PhD Dissertation.

Wang, Y., Nnaji, B.O., (2005). Geometry-Based Semantic ID for Persistent and Interoperable Reference in Feature-Based Parametric Modeling. Computer-Aided Design, vol. 37(10), pp. 1081-1093

Whittenburg, M., (2012). Model-based enterprise: an innovative technology-enabled contract management approach. Journal of Contract Management, vol. 10, pp. 103112

Wolfe, J.M., (1994). Guided Search 2.0: A Revised Model of Visual Search. Psychonomic Bulletin \& Review, vol. 1(2), pp. 202-238

Woodruff, A., Landay, J., Stonebraker, M., (1998). Constant Information Density in Zoomable Interfaces. Working Conference on Advanced Visual Interfaces, pp. 5765

Yang, J., Han, S., (2006). Repairing CAD model errors based on the design history. Computer Aided Design, vol. 38(6), pp. 627-640

You, C.F., Chao, S.N., (2006). Multilayer architecture in collaborative environment. Concurrent Engineering Research and Applications, vol. 14(4), pp. 273-281 



\section{APPENDIX A: PART ALTERATION. ACTIVITY 1}

Part modification instructions for participants:

1. Modify the given bracket by creating a second arm, identical to the existing one, on the other side of the part. See figure below.

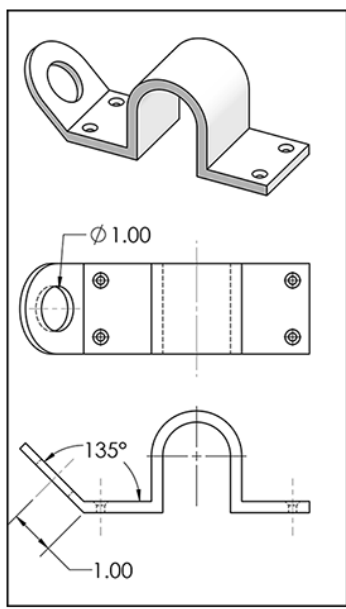

ORIGINAL MODEL

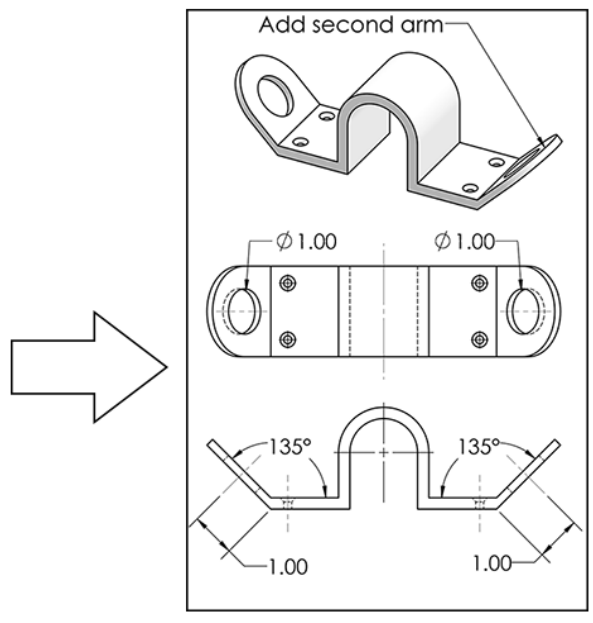

ALTERED MODEL

2. Change the diameter of the $1.00 "$ hole in the new arm to 0.5 " Maintain the $1.00 "$ diameter for the hole located in the original arm. See figure below.
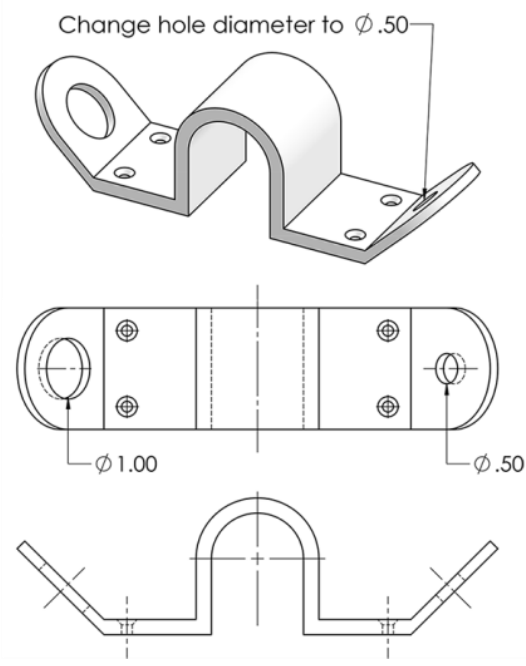
Annotation Mechanisms to Manage Design Knowledge in Complex Parametric Models and their Effects on Alteration and Reusability

3. Change the new arm you created, according to the dimensions shown in the figure below.
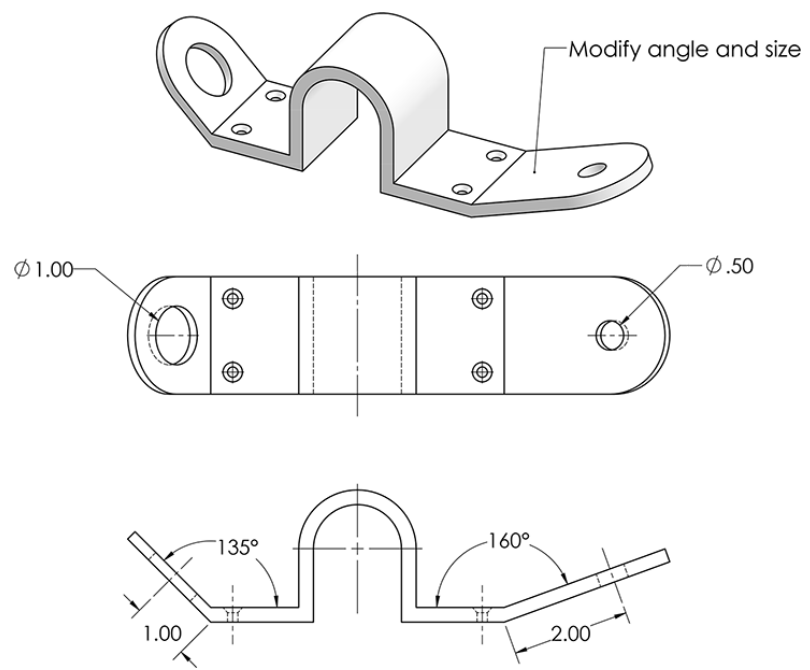


\section{APPENDIX B: PART ALTERATION. ACTIVITY 2}

Part modification instructions for participants:
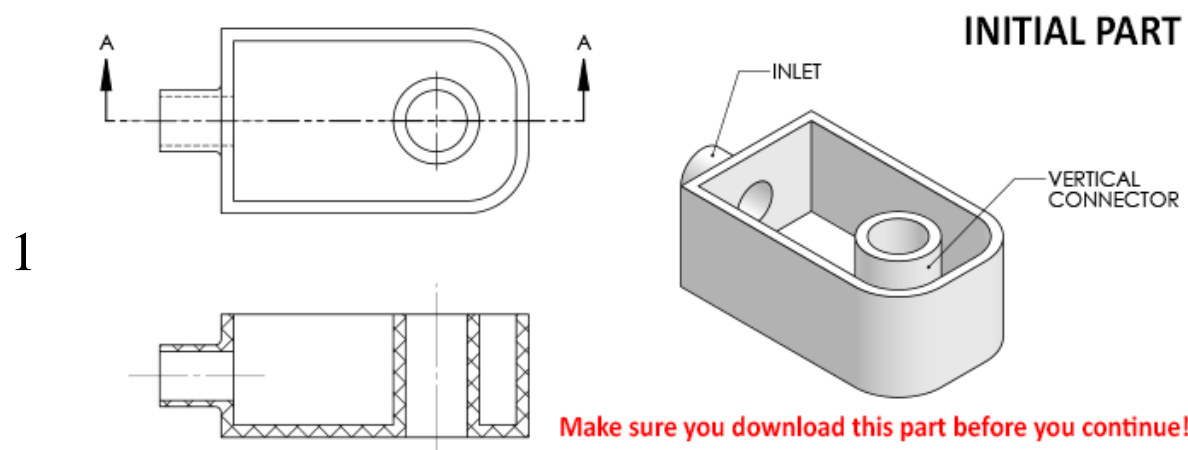

Make sure you download this part before you continue!

SECTION A-A

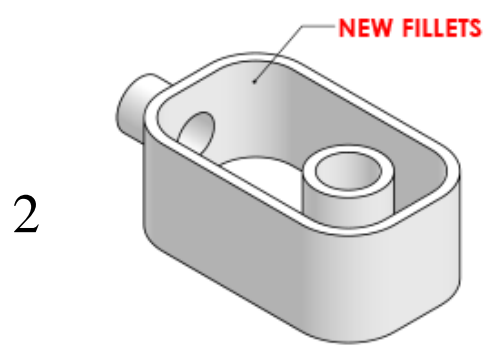

CREATE FILLETS ON OPPOSITE SIDE (Equal radii)
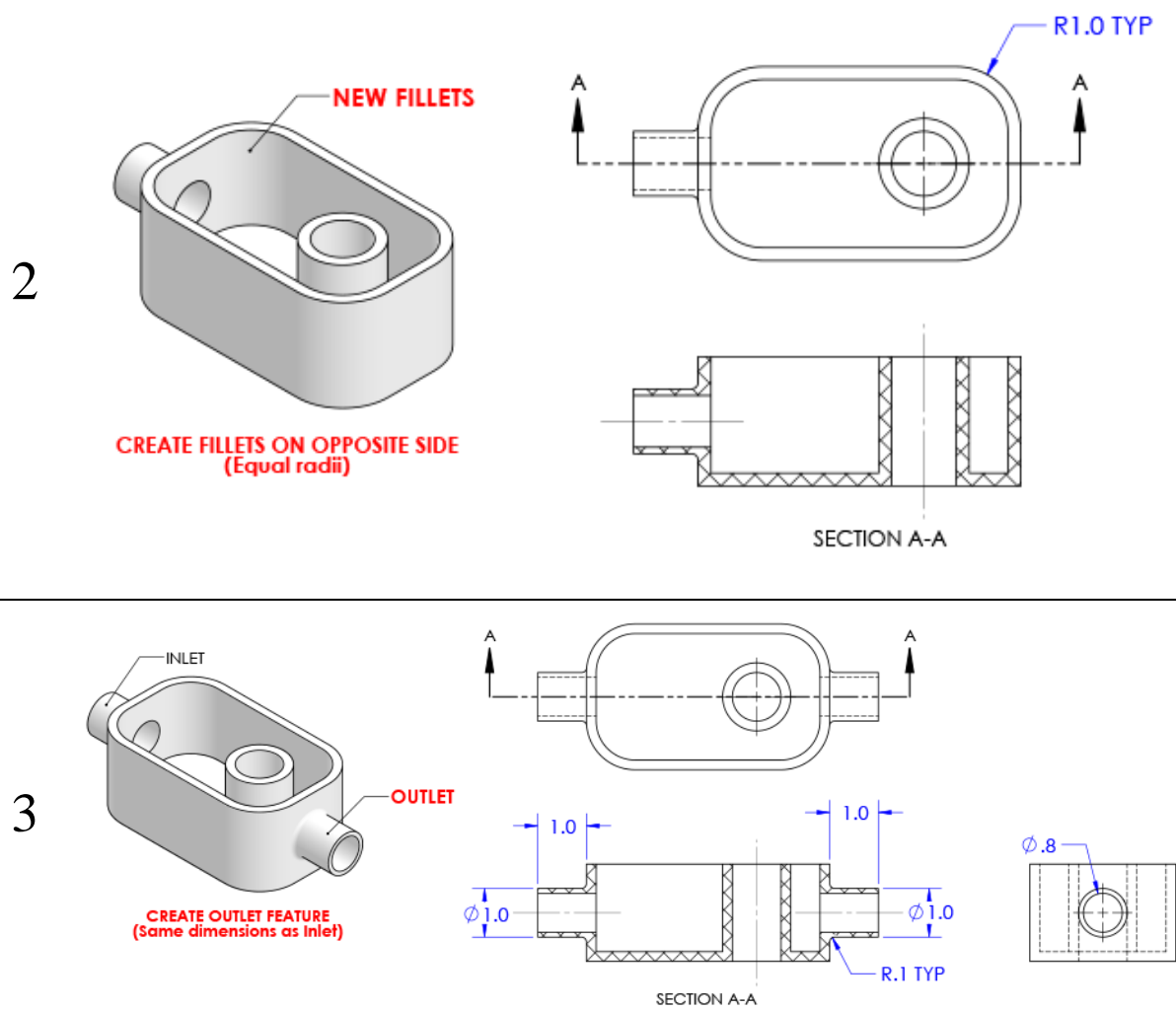

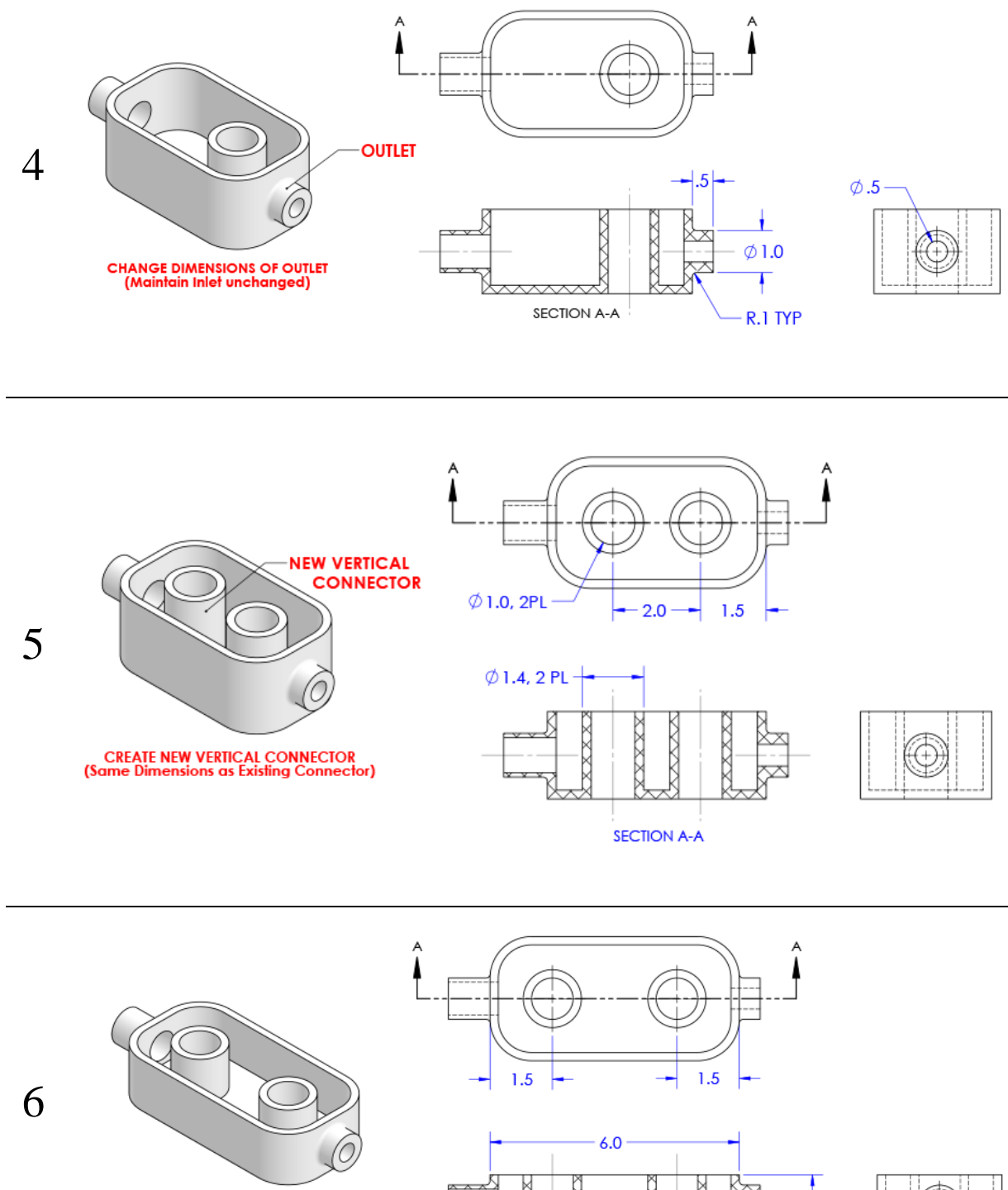

CHANGE OVERALL WIDTH AND HEIGHT OF THE PART
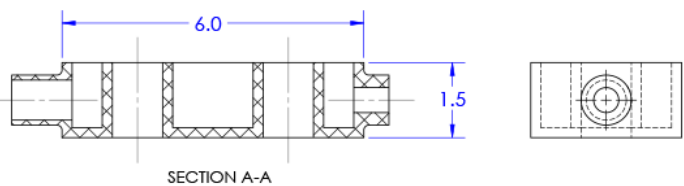


\section{APPENDIX C: EXPERIMENTS 1 AND 2}

(Correct answers are provided in italic letters as a reference)

\section{$\underline{\text { Instructions }}$}

You have been given the task to make a number of modifications to an existing 3D model ("bracket.SLDPRT") and find certain information. This 3D model has been annotated to provide design and modeling information within the geometry. During the annotation process, multiple designers have collaborated to provide a variety of data based on design requirements, test results, revisions, previous experience, etc.

As in many other cases in parametric modeling, each modification that needs to be performed can be accomplished in different ways. However, previous knowledge and experience from the team of designers and engineers have helped determine the most effective approaches. This information has been captured and included within the model in the form of $3 \mathrm{D}$ annotations.

Question 1: According to the annotations, if you want to modify the size of the M4 machine holes located on the base of the model, you must first:
a) Your answer:
b) I wasn't able to find enough information in the annotations to answer confidently.

Correct answer: Contact Design Center

Answer is provided by the following model annotation(s):

- “Contact Design Center if modifying M4 holes” connected to Feature "M4x0.7 Tapped Hole 1"

Question 2: According to the annotations, what is the range of acceptable angles for the feature shown below?

a) Min angle $=$ Max angle =

b) I wasn't able to find enough information in the annotations to answer confidently.

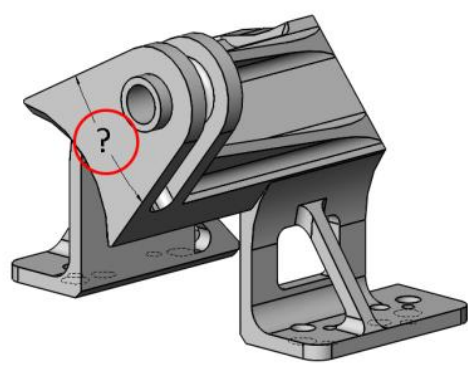


Annotation Mechanisms to Manage Design Knowledge in Complex Parametric Models and their Effects on Alteration and Reusability

Correct answer: Min angle $=20^{\circ}$ and Max angle $=27^{\circ}$

Answer is provided by the following model annotation(s):

- "Ensure a minimum angle of 20 degrees" connected to Feature "BossExtrude3"

- "Ensure a maximum angle of 27 degrees" connected to Feature "BossExtrude3"

Question 3: According to the annotations, the most effective procedure to create the second rib on the back of the model (on the available side) as shown in the figure below is:

a) Use Mirror about a center vertical plane to create a symmetrical image of the first rib

b) Create a new sketch on the inner flat surface and extrude

c) Use linear pattern using the original rib as seed to create the second rib

d) Use the existing sketch of the existing rib to create a new extrusion with an offset distance

e) I wasn't able to find enough information in the annotations to answer confidently.

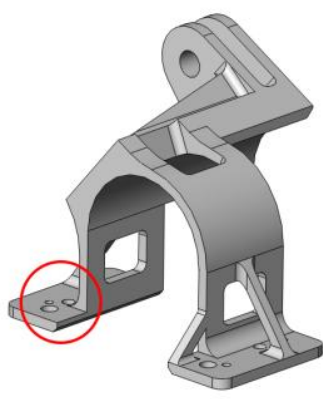

ORIGINAL

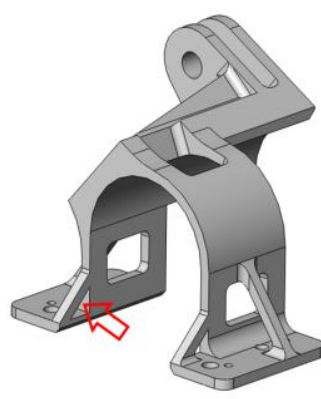

ALTERED

Correct answer: $B$

Answer is provided by the following model annotation(s):

- "Do not use symmetry or pattern for ribs. Angles may vary" connected to Feature "Boss-Extrude9" 


\section{Question 4: When modifying the dimensions of the side ribs to $5 \mathrm{~mm} \times 5 \mathrm{~mm}$} (left figure below), some errors and unwanted effects occur in the model, such as the one shown in the right figure below. According to the annotations, the most effective procedure to resolve these effects is:

a) Reduce the height of the rectangular cut (Cut-Extrude1) to $12 \mathrm{~mm}$ or less so it doesn't interfere with the modified rib.

b) Move the rectangular cut down, so that the new distance from the bottom of the cut to the bottom of the part is $8 \mathrm{~mm}$. Then, reduce the radius of Fillet7 to $3 \mathrm{~mm}$. Delete Fillet11.

c) Increase the angle of the ribs.

d) Because of preliminary FEA results, the new dimensions of the side ribs $(5 \mathrm{~mm} \times 5 \mathrm{~mm})$ are not allowed.

e) I wasn't able to find enough information in the annotations to answer confidently.
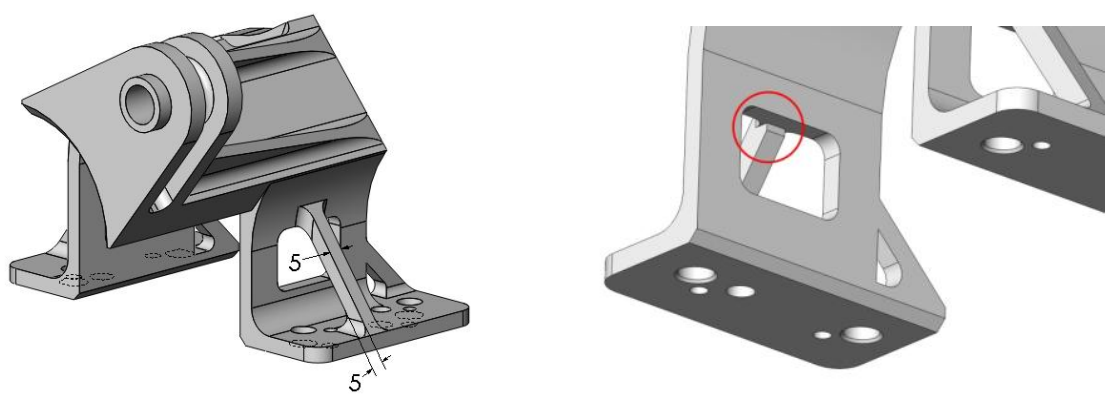

Correct answer: $B$

Answer is provided by the following model annotation(s):

- "Optimum rib angle. Maintain in future versions" connected to Feature "Boss-Extrude8”

- " 3.00 x 3.00 Minimum cross section defined by FEA analysis" connected to Feature "Boss-Extrude8"

- "Keep dimensions of the cut constant. Modify position if necessary" connected to Feature "Cut-Extrude1" 